\title{
THE EFFECT OF LINEAR PERTURBATIONS ON THE YAMABE PROBLEM
}

\author{
PIERPAOLO ESPOSITO, ANGELA PISTOIA, AND JÉRÔME VÉTOIS
}

\begin{abstract}
In conformal geometry, the Compactness Conjecture asserts that the set of Yamabe metrics on a smooth, compact, aspherical Riemannian manifold $(M, g)$ is compact. Established in the locally conformally flat case by Schoen 43, 44 and for $n \leq 24$ by KhuriMarques-Schoen [26, it has revealed to be generally false for $n \geq 25$ as shown by Brendle [8] and Brendle-Marques 9. A stronger version of it, the compactness under perturbations of the Yamabe equation, is addressed here with respect to the linear geometric potential $\frac{n-2}{4(n-1)} \mathrm{Scal}_{g}$, Scal $_{g}$ being the Scalar curvature of $(M, g)$. We show that a-priori $L^{\infty}$-bounds fail for linear perturbations on all manifolds with $n \geq 4$ as well as a-priori gradient $L^{2}$-bounds fail for nonlocally conformally flat manifolds with $n \geq 6$ and for locally conformally flat manifolds with $n \geq 7$. In several situations, the results are optimal. Our proof combines a finite dimensional reduction and the construction of a suitable ansatz for the solutions generated by a family of varying metrics in the conformal class of $g$.
\end{abstract}

\section{INTRODUCTION}

Letting $(M, g)$ be a smooth, compact Riemannian $n$-manifold, $n \geq 3$, we are concerned with the so-called Yamabe equation

$$
\Delta_{g} u+\alpha_{n} \operatorname{Scal}_{g} u=c u^{2^{*}-1}, \quad u>0 \quad \text { in } M,
$$

where $\Delta_{g}:=-\operatorname{div}_{g} \nabla$ is the Laplace-Beltrami operator, $\alpha_{n}:=\frac{n-2}{4(n-1)}$, Scal $_{g}$ is the Scalar curvature of the manifold, $2^{*}=\frac{2 n}{n-2}$ is the critical Sobolev exponent, and $c \in \mathbb{R}$. The geometric problem of finding a metric $\widetilde{g}$ in the conformal class $[g]=\left\{\phi g: \phi \in C^{\infty}(M), \phi>0\right\}$ of $g$ with constant Scalar curvature is equivalent to solving (1.1) through the setting $\widetilde{g}=u^{4 /(n-2)} g$. The constant $c$ can be restricted to the values $-1 / 1$ or 0 depending on whether the Yamabe invariant

$$
\mu_{g}(M)=\inf _{\widetilde{g} \in[g]}\left(\operatorname{Vol}_{\widetilde{g}}(M)^{\frac{2-n}{n}} \int_{M} \operatorname{Scal}_{\widetilde{g}} d v_{\widetilde{g}}\right)
$$

of $(M, g)$ has negative/positive sign or vanishes, respectively, where $\operatorname{Vol}_{\widetilde{g}}(M)$ is the volume of the manifold $(M, \widetilde{g})$.

The Yamabe problem, raised by H. Yamabe [49] in '60, was firstly solved by Trudinger [48] when $\mu_{g}(M) \leq 0$. In this case, the solution is unique (up to a normalization when $\mu_{g}(M)=0$ ). Aubin [3] then solved the Yamabe problem in the non-locally conformally flat (non-l.c.f. for short) case with $n \geq 6$, and Schoen [42] solved it in the remaining cases of low dimensions $3 \leq n \leq 5$ and locally conformally flat (l.c.f. for short) manifolds. In this paper, we restrict our attention to the case where $(M, g)$ has positive Yamabe invariant $\mu_{g}(M)>0$, and we set $c=1$ in (1.1).

Date: June 24, 2018.

The first author was partially supported by the Prin project "Critical Point Theory and Perturbative Methods for Nonlinear Differential Equations" and the Firb-Ideas project "Analysis and Beyond". The third author was partially supported by the ANR grant ANR-08-BLAN-0335-01. 
After the complete resolution of the Yamabe problem, one can attempt to describe the solution set of (1.1). A well-known conjecture claims the compactness of Yamabe metrics for manifolds $(M, g)$ which are not conformally equivalent to $\left(\mathbb{S}^{n}, g_{0}\right)\left((M, g) \neq\left(\mathbb{S}^{n}, g_{0}\right)\right.$ for short), namely the convergence, up to a subsequence, in $C^{2}(M)$ of any sequence of solutions of equation (1.1). Referred to in literature as the Compactness Conjecture, by elliptic regularity theory, it amounts to prove a-priori $L^{\infty}$-bounds on the set of solutions to (1.1). In the basic example of the round sphere $\left(\mathbb{S}^{n}, g_{0}\right)$, by the works of Lelong-Ferrand [28] and Obata [36], the set of solutions of (1.1) is explicit and non-compact. The Compactness Conjecture arose after the work of Schoen [43, 44] who first proved it in the l.c.f. case, and also proposed a strategy, based on the Pohozaev identity, for proving it in the non-l.c.f. case. The Compactness Conjecture has been then proved in the low-dimensional case by $\mathrm{Li}-\mathrm{Zhu}[32$ for $n=3$, by Druet [14 for $n \leq 5$, by Marques [33] for $n \leq 7$, by Li-Zhang [30,31] for $n \leq 11$, and finally, by Khuri-Marques-Schoen [26] for $n \leq 24$. Unexpectedly, the dimension $n=24$, which arises in [26] as the maximal dimension for a suitable quadratic form to be positive definite, has revealed to be optimal by the counter-examples constructed in dimensions $n \geq 25$ by Brendle [8] and Brendle-Marques [9]. We also refer to the constructions by Ambrosetti-Malchiodi [1] and Berti-Malchiodi [7] in case of background metrics which have a finite regularity. All these constructions are made on $\left(\mathbb{S}^{n}, g\right)$ with a metric $g$ close to the round one $g_{0}$.

Replacing the geometric potential $\alpha_{n} \mathrm{Scal}_{g}$ in (1.1) with a general potential $\kappa \in C^{1}(M)$ such that $\kappa(\xi) \neq \alpha_{n} \operatorname{Scal}_{g}(\xi)$ for all $\xi \in M$, the Compactness Conjecture is esentially still true for solutions with bounded energy of

$$
\Delta_{g} u+\kappa u=u^{2^{*}-1}, \quad u>0 \quad \text { in } M,
$$

provided that $n \geq 4$, as shown by Druet [13, Section 4].

Towards a deeper understanding of the Yamabe equation, one can address a stronger version of the Compactness Conjecture. One can ask whether or not the compactness property is preserved under perturbations of the equation under exam, which is equivalent to have or not uniform a-priori $L^{\infty}$-bounds for solutions of the perturbed problem. This question has been introduced and investigated in Druet [13, 14, Druet-Hebey [15, 16], Druet-Hebey-Robert [18], and, under the name of stability, in Druet-Hebey [17] and Druet-Hebey-Vetois [19]. The aim of our paper is to establish non-compactness properties for linear perturbations of the geometric potential $\alpha_{n} \mathrm{Scal}_{g}$ in (1.1). In case $3 \leq n \leq 5$, Druet [14 obtained uniform $L^{\infty}$ bounds for solutions of (1.2) along potentials $\kappa_{\varepsilon} \leq \alpha_{n} \mathrm{Scal}_{g}, \kappa_{\varepsilon} \rightarrow \kappa$ in $C^{2}(M)$ as $\varepsilon \rightarrow 0$, with $(M, g) \neq\left(\mathbb{S}^{n}, g_{0}\right)$ in case $\kappa=\alpha_{n} \mathrm{Scal}_{g}$. The same result is strongly expected to be true in the l.c.f. case and generally for $n \leq 24$, and it is still true, as shown by Druet [14], when $n \geq 6$ and $\kappa<\alpha_{n}$ Scal $_{g}$. In dimension $n=3, \mathrm{Li}-\mathrm{Zhu}$ [32, Theorem 0.3] have obtained uniform $L^{\infty}$-bounds in case $\kappa_{\varepsilon} \leq \alpha_{n} \mathrm{Scal}_{g}+\eta_{0}$, for some $\eta_{0}>0$ when $(M, g) \neq\left(\mathbb{S}^{n}, g_{0}\right)$.

Let us briefly review the previous results of non-compactness for equations of type (1.2). Apart from the trivial case of the Yamabe equation on $\left(\mathbb{S}^{n}, g_{0}\right)$, the first non-compactness result is due to Hebey-Vaugon [24] who proved that in the l.c.f. case with $n \geq 4$, there always exists $\widetilde{g} \in[g]$ such that the equation $\Delta_{\widetilde{g}} u+\alpha_{n} \max _{M}\left(\operatorname{Scal}_{\widetilde{g}}\right) u=u^{2^{*}-1}$ in $M$ is not compact. Families of non-compact solutions have then been explicitly constructed on $\left(\mathbb{S}^{n}, g_{0}\right)$ by Druet [13] and Druet-Hebey [15] for linear perturbations of the potential $\kappa=\alpha_{n}$ Scal $_{g}$ when $n \geq 6$ and in case $\kappa>\alpha_{n}$ Scal $_{g}$ when $n=6$ (see also the survey paper by Druet-Hebey [16] for the case of quotients of $\left.\left(\mathbb{S}^{n}, g_{0}\right)\right)$. When $\left(\kappa-\alpha_{n} \mathrm{Scal}_{g}\right)$ is a positive constant, on $(M, g)=\left(\mathbb{S}^{n}, g_{0}\right)$, Chen-Wei-Yan [12] have constructed infinitely many solutions with unbounded energy when $n \geq 5$, and Hebey-Wei [25] have constructed non-compact solutions with bounded energy for 
an infinite number of constant $\kappa$ in case $n=3$. Concerning the potential $\kappa=\alpha_{n} \mathrm{Scal}_{g}$, so far, the only available examples of non-compact solutions for (1.1) and its linear perturbations are in the case of $\left(\mathbb{S}^{n}, g\right)$, with $g$ close to $g_{0}$ and $\kappa=\alpha_{n}$ Scal $_{g}$ by Ambrosetti-Malchiodi [1], BertiMalchiodi [7], Brendle [8], and Brendle-Marques [9], or with $g=g_{0}$ and $\kappa$ close to $\alpha_{n}$ Scal $_{g_{0}}$ by Druet [13] and Druet-Hebey [15] (see also Druet-Hebey [16] for the case of quotients of $\left.\left(\mathbb{S}^{n}, g_{0}\right)\right)$.

In this paper, for $n \geq 4$, we exhibit the general failure of compactness properties for

$$
\Delta_{g} u+\left(\alpha_{n} \operatorname{Scal}_{g}+\varepsilon h\right) u=u^{2^{*}-1}, \quad u>0 \quad \text { in } M,
$$

where $h$ is a $C^{1}$ or $C^{0, \alpha}$-function in $M, \alpha \in(0,1)$, with $\max _{M} h>0$ and $\varepsilon>0$ is a small parameter. As a by-product, we obtain that the Compactness Conjecture completely fails down under the effect of linear perturbations (with the correct sign) of the Yamabe equation (1.1) on every manifold $(M, g)$ with $n \geq 4$ (but it is still true for $n=3$ by Li-Zhu [32]). Our results, together with those by Druet [13, 14, give a sharp picture of the situation. Even more than the failure of a-priori $L^{\infty}$-bounds, we show that a-priori gradient $L^{2}$-bounds fail for non-locally conformally flat manifolds with $n \geq 6$ and for locally conformally flat manifolds with $n \geq 7$.

To be more precise, we say that a family $\left(u_{\varepsilon}\right)_{\varepsilon}$ of solutions to equation (1.3) blows up at some point $\xi_{0} \in M$ if there holds $\sup _{U} u_{\varepsilon} \rightarrow+\infty$ as $\varepsilon \rightarrow 0$, for all neighborhoods $U$ of $\xi_{0}$ in $M$. Let $E: M \rightarrow(-\infty,+\infty]$ be defined as

$$
E(\xi)= \begin{cases}h(\xi) A_{\xi}^{-\frac{2}{n-2}} & \text { if } n=4,5 \text { or }(M, g) \text { l.c.f. } \\ h(\xi) \mid \text { Weyl }\left._{g}(\xi)\right|_{g} ^{-1} & \text { if } n \geq 6 \text { and }(M, g) \text { non-l.c.f. }\end{cases}
$$

with the convention that $1 / 0=+\infty$. Here, $A_{\xi}$ is defined in (1.5) and Weyl $g$ is the Weyl curvature tensor of the manifold. In dimensions $n=3,4,5$ or if the manifold is l.c.f., up to a conformal change of metric (depending on $\xi \in M$ ), the Green's function $G_{g}(\cdot, \xi)$ has an asymptotic expansion of the form

$$
G_{g}\left(\exp _{\xi} y, \xi\right)=\beta_{n}^{-1}|y|^{2-n}+A_{\xi}+\mathrm{O}(|y|)
$$

as $y \rightarrow 0$, where $\beta_{n}:=(n-2) \omega_{n-1}, \omega_{n-1}$ is the volume of the unit $(n-1)$-sphere, and $A_{\xi} \in \mathbb{R}$, see Lee-Parker [27]. The geometric quantity $A_{\xi}$ depends only on the manifold $(M, g)$ and the point $\xi$, is smooth with respect to $\xi$, and can be identified with the mass of a stereographic projection of the manifold with respect to $\xi$. We refer to Lee-Parker [27] for the definition of the mass and a discussion about its role in general relativity. In particular, for manifolds $(M, g) \neq\left(\mathbb{S}^{n}, g_{0}\right)$, in case $n=4,5$ and in the l.c.f. case with $n \geq 6$, we have that $A_{\xi}>0$ by the result of Schoen-Yau [45, 46], and thus $E(\xi)<+\infty$ for all $\xi \in M$. In the non-l.c.f. case with $n \geq 6$, we have that Weyl $_{g} \not \equiv 0$, and thus $E(\xi) \not \equiv+\infty$. Our first result concerns the existence of solutions blowing-up at one point and reads as:

Theorem 1.1. (existence of solutions with a single blow-up point in case $n \geq 4$ ) Let $(M, g) \neq\left(\mathbb{S}^{n}, g_{0}\right)$ be a smooth compact Riemannian manifold with $n \geq 4$ and $\mu_{g}(M)>0$, and $h \in C^{0, \alpha}(M), \alpha \in(0,1)$, be so that $\max _{M} h>0$. In the non-l.c.f. case with $n \geq 6$, assume in addition that $\min \left\{\left|\operatorname{Weyl}_{g}(\xi)\right|_{g}: h(\xi)>0\right\}>0$. Then for $\varepsilon>0$ small, equation (1.3) has a solution $u_{\varepsilon} \in C^{2, \alpha}(M)$ such that the family $\left(u_{\varepsilon}\right)_{\varepsilon}$ blows up, up to a sub-sequence, as $\varepsilon \rightarrow 0$ at some point $\xi_{0}$ so that $E\left(\xi_{0}\right)=\max _{M} E$.

Let us mention that the Compactness Conjecture does hold for (1.1) when $n \geq 6$ as soon as $\mid$ Weyl $_{g}(\xi) \mid>0$ for all $\xi \in M$, as it follows by Li-Zhang [30] and Marques [33]. The following result concerns multiplicity of solutions with a single blow-up point. Isolated critical points of 
$E$ with non-trivial local degree include non-degenerate critical points of $E$. The result reads as:

Theorem 1.2. (multiplicity of solutions with a single blow-up point in case $n \geq 4$ ) Let $(M, g) \neq\left(\mathbb{S}^{n}, g_{0}\right)$ be a smooth compact Riemannian manifold with $n \geq 4$ and $\mu_{g}(M)>0$, and $h \in C^{1}(M)$. For any isolated critical point $\xi_{0}$ of $E$ with non-trivial local degree and $h\left(\xi_{0}\right)>0$, for $\varepsilon>0$ small, equation (1.3) has a solution $u_{\varepsilon} \in C^{2, \alpha}(M), \alpha \in(0,1)$, such that the family $\left(u_{\varepsilon}\right)_{\varepsilon}$ blows up, up to a sub-sequence, at $\xi_{0}$ as $\varepsilon \rightarrow 0$.

As already said, by the result of Li-Zhu [32, such blowing-up solutions as in Theorems 1.1 and 1.2 do not exist in dimension $n=3$. The last results, Theorems 1.3 , 1.4, and 1.5 below, claim the existence of solutions which blow up at more than one point. The first result concerns the non-l.c.f. case with $n \geq 6$ and reads as:

Theorem 1.3. (existence of solutions with more than one blow-up point in the non-l.c.f. case with $n \geq 6)$ Let $(M, g)$ be a smooth compact non-l.c.f. Riemannian manifold with $n \geq 6$ and $\mu_{g}(M)>0$. Let $k \geq 2$ be an integer and $h_{k} \in C^{0, \alpha}(M), \alpha \in(0,1)$, be so that the set $\left\{\xi \in M: h_{k}(\xi)>0\right\}$ has $k$ connected components $\mathcal{C}_{1}, \ldots, \mathcal{C}_{k}$ and $\min \left\{\left|\operatorname{Weyl}_{g}(\xi)\right|_{g}\right.$ : $h(\xi) \geq 0\}>0$. Then, for $\varepsilon>0$ small, equation (1.3) has a solution $u_{k, \varepsilon} \in C^{2, \alpha}(M)$ such that the family $\left(u_{k, \varepsilon}\right)_{\varepsilon}$ blows up, up to a sub-sequence, as $\varepsilon \rightarrow 0$ at $k$ distinct points $\left(\xi_{0}\right)_{1}, \ldots,\left(\xi_{0}\right)_{k}$ so that

$$
\frac{h_{k}\left(\left(\xi_{0}\right)_{j}\right)}{\left|\operatorname{Weyl}_{g}\left(\left(\xi_{0}\right)_{j}\right)\right|_{g}}=\max _{\xi \in \mathcal{C}_{j}} \frac{h_{k}(\xi)}{\left|\operatorname{Weyl}_{g}(\xi)\right|_{g}}
$$

for all $j=1, \ldots, k$. Moreover, there holds $\lim _{k \rightarrow+\infty} \lim \sup _{\varepsilon \rightarrow 0}\left\|\nabla u_{k, \varepsilon}\right\|_{L^{2}(M)}=+\infty$.

Each blow-up point $\left(\xi_{0}\right)_{j}, j=1, \ldots, k$, in Theorem 1.3 maximizes the same function $E$ as in Theorem 1.1 for single blow-up points. On the contrary, in the remaining cases, there is a strong interaction between the blow-up points, and these are not anymore related to maximum points of the function $E$ defined in (1.4). Concerning the l.c.f. case with $n \geq 7$, we prove the following result:

Theorem 1.4. (existence of solutions with more than one blow-up point in the l.c.f. case with $n \geq 7)$ Let $(M, g) \neq\left(\mathbb{S}^{n}, g_{0}\right)$ be a smooth compact l.c.f. Riemannian manifold with $n \geq 7$ and $\mu_{g}(M)>0$, and $h \in C^{0, \alpha}(M), \alpha \in(0,1)$, be so that $\max _{M} h>0$. Then for any integer $k \geq 2$, for $\varepsilon>0$ small, equation (1.3) has a solution $u_{k, \varepsilon} \in C^{2, \alpha}(M)$ such that the family $\left(u_{k, \varepsilon}\right)_{\varepsilon}$ blows up, up to a sub-sequence, at $k$ distinct points $\left(\xi_{0}\right)_{1}, \ldots,\left(\xi_{0}\right)_{k}$ in $M$ as $\varepsilon \rightarrow 0$. Moreover, there holds $\lim _{k \rightarrow+\infty} \lim \sup _{\varepsilon \rightarrow 0}\left\|\nabla u_{k, \varepsilon}\right\|_{L^{2}(M)}=+\infty$.

The location of $\xi_{0}=\left(\left(\xi_{0}\right)_{1}, \ldots,\left(\xi_{0}\right)_{k}\right)$ in Theorem 1.4 is related to maximum points of a "reduced energy" given in (5.1), and the assumption $n \geq 7$ guarantees that such "reduced energy" achieves its maximum value.

Our last result, Theorem 1.5 below, concerns the l.c.f. case for $n=6$. This case reveals to be even more intricate than the case of higher dimensions. For any integer $k \geq 2$, define $\Delta_{k}:=\left\{\left(\xi_{1}, \ldots, \xi_{k}\right) \in M^{k}: \xi_{i}=\xi_{j}\right.$ for $\left.i \neq j\right\}$. For any $\boldsymbol{\xi}:=\left(\xi_{1}, \ldots, \xi_{k}\right) \in M^{k} \backslash \Delta_{k}$, let $A_{k, \boldsymbol{\xi}}$ be the symmetric $k \times k$ matrix with entries

$$
\left(A_{k, \boldsymbol{\xi}}\right)_{i j}:= \begin{cases}A_{\xi_{i}} & \text { if } i=j \\ G_{g}\left(\xi_{i}, \xi_{j}\right) & \text { if } i \neq j\end{cases}
$$

where $A_{\xi_{i}}$ is as in (1.5). When $A_{k, \boldsymbol{\xi}}$ is invertible, let $E_{k}: M^{k} \backslash \Delta_{k} \rightarrow \mathbb{R}$ be defined as

$$
E_{k}(\boldsymbol{\xi}):=\left\langle H(\boldsymbol{\xi}), A_{k, \boldsymbol{\xi}}^{-1} \cdot H(\boldsymbol{\xi})\right\rangle,
$$


where $H(\boldsymbol{\xi}):=\left(h\left(\xi_{1}\right), \ldots, h\left(\xi_{k}\right)\right)$ and $\langle\cdot, \cdot\rangle$ is the Euclidean scalar product. Here, contrary to the situation with $k=1$, the definition of $E_{k}(\boldsymbol{\xi})$ allows to consider the case $(M, g)=\left(\mathbb{S}^{n}, g_{0}\right)$. In this case, $A_{k, \boldsymbol{\xi}}$ has all entries equal to zero on the diagonal. In particular, when $k=2$ or

3 , observe that $A_{k, \boldsymbol{\xi}}$ is invertible, and thus $E_{k}(\boldsymbol{\xi})$ is well-defined, for all $\boldsymbol{\xi} \in\left(\mathbb{S}^{n}\right)^{k} \backslash \Delta_{k}$. Our result in the l.c.f. case with $n=6$ reads as:

Theorem 1.5. (existence of solutions with more than one blow-up point in the l.c.f. case with $n=6)$ Let $(M, g)$ be a smooth compact l.c.f. Riemannian manifold with $n=6$ and $\mu_{g}(M)>0$, and $h \in C^{1}(M)$. Let $k \geq 2$ be an integer, and assume that $E_{k}$ has an isolated critical point $\boldsymbol{\xi}_{0}:=\left(\left(\xi_{0}\right)_{1}, \ldots,\left(\xi_{0}\right)_{k}\right)$ with non-trivial local degree and $A_{k, \boldsymbol{\xi}_{0}}^{-1} \cdot H\left(\boldsymbol{\xi}_{0}\right)$ has positive coordinates. Then for $\varepsilon>0$ small, equation (1.3) has a solution $u_{k, \varepsilon} \in C^{2, \alpha}(M)$, $\alpha \in(0,1)$, such that the family $\left(u_{k, \varepsilon}\right)_{\varepsilon}$ blows up, up to a sub-sequence, at $\left(\xi_{0}\right)_{1}, \ldots,\left(\xi_{0}\right)_{k}$ as $\varepsilon \rightarrow 0$.

Contrary to the assumptions in the previous theorems, here it seems unclear in general when the function $E_{k}$ admits an isolated critical point with non-trivial local degree. An easy situation where we can construct $h$ and $\boldsymbol{\xi}_{0}$ satisfying the assumptions in Theorem 1.5 is the case $k=2$ on the round sphere $\left(\mathbb{S}^{n}, g_{0}\right)$. Indeed, in this case, we find that $E_{2}(\boldsymbol{\xi})=$ $2 h\left(\xi_{1}\right) h\left(\xi_{2}\right) G_{g_{0}}\left(\xi_{1}, \xi_{2}\right)^{-1}$ for all $\boldsymbol{\xi}=\left(\xi_{1}, \xi_{2}\right), \xi_{1} \neq \xi_{2}$, which has always a maximum point in $\left(\mathbb{S}^{n} \cap\{h \geq 0\}\right)^{2} \backslash \Delta_{2}$ provided that $\max _{M} h>0$. It is clear that the maximum point is non-degenerate for several $h^{\prime}$ s (in a generic sense).

Let us finally compare problem (1.3) with its Euclidean counter-part on a smooth bounded domain $\Omega \subset \mathbb{R}^{n}, n \geq 4$, with homogeneous Dirichlet boundary condition

$$
\Delta_{\text {Eucl }} u+\lambda u=u^{2^{*}-1} \text { in } \Omega, \quad u>0 \text { in } \Omega, \quad u=0 \text { on } \partial \Omega .
$$

For $\lambda \geq 0$, a direct minimization method (for the corresponding Rayleigh quotient) never gives rise to any solution of (1.8), and moreover, no solution exists at all if $\Omega$ is star-shaped as shown by Pohožaev [38. Moreover, following the arguments developed by Ben AyedEl Mehdi-Grossi-Rey [5], problem (1.8) has no solutions with a single blow-up point as $\lambda \rightarrow 0^{+}$. The effect of the geometry, which is crucial to provide a solution for the Yamabe problem (corresponding to $\lambda=0$ in (1.8) ) by minimization, is also relevant to producing solutions of (1.3) (corresponding to $\lambda \rightarrow 0^{+}$in (1.8)) with a single blow-up point as stated in Theorems 1.1 and 1.2. On the contrary, Theorem 1.4 has a partial counter-part on domains with nontrivial topology, see Musso-Pistoia [34] and Pistoia-Rey [37]. When $\lambda<0$, solutions of (1.8) can be found by direct minimization as shown by Brezis-Nirenberg [10], and exhibit a single blow-up point as $\lambda \rightarrow 0^{-}$as shown by Han [23], in contrast with the compactness property proved by Druet 13. Solutions of (1.8) with a single blow-up point, see Rey [39, 40, and with multiple blow-up points, see Bahri-Li-Rey [4] and Musso-Pistoia [35], as $\lambda \rightarrow 0^{-}$have been constructed in a very general way. Since the manifold with boundary $(\Omega, d x)$ is l.c.f., notice that the Green's function $G(\cdot, \xi)$ still has an asymptotic expansion of the form (1.5), but the constant $A_{\xi}$ is always negative in this case. The different sign of $A_{\xi}$ is the analytical reason of the completely different picture we have for equations (1.3) and (1.8).

The paper is organized in the following way. In Section 2 we describe the perturbative method we use to attack existence issues of blowing-up solutions. We describe the main steps of such an approach, leading to the general result Theorem 2.4, and we deduce from it our main results concerning solutions with a single blow-up point. A crucial point is to produce a suitable ansatz for the solutions. Inspired by the approach of Lee-Parker [27], which unifies the previous constructions of Aubin [3] and Schoen [42] in the resolution of the Yamabe problem, 
we build up general approximating solutions $W_{\varepsilon, t, \xi}$ for (1.3) which approximation rates are evaluated in Section 3. An important point here is that we allow the metric $g$ to vary in the conformal class so to gain flatness at each point $\xi \in M$. An alternative, less geometric approach can be devised in the non-l.c.f. case, see Esposito-Pistoia-Vétois [20], by keeping $g$ fixed and slightly correcting the basic ansatz via linearization so to account for the local geometry. Thanks to the solvability theory of the linearized operator for (1.3) at $W_{\varepsilon, t, \xi}$, we are led to study critical points of a finite-dimensional functional $\mathcal{J}_{\varepsilon}(t, \xi)$. A key step is to obtain an asymptotic expansion of $\mathcal{J}_{\varepsilon}(t, \xi)$ and to identify a "reduced energy" as the main order term. This step is performed in Section 4 in $C^{0}$-sense and completes the proof of Theorem 1.1. In Section 5, we investigate the existence of solutions with $k$ blow-up points, yielding to the proofs of Theorems 1.3, 1.4, and 1.5. The $C^{1}$-expansion of $\mathcal{J}_{\varepsilon}(t, \xi)$ is addressed in Section 6, completing the proof of Theorem 1.2. The appendix is devoted to some technical issues.

Acknowledgments: this work has been initiated and partially carried out during the visits of the third author to the University of "Roma La Sapienza" in November 2009 and to the University of "Roma Tre" in November 2010. The third author gratefully acknowledges the hospitality and the financial support of these two institutions.

\section{SCHEME OF THE PROOF}

In this section, we aim to give the scheme of proof for Theorem 2.4 below. First, let us set some notations. We denote the conformal Laplacian of the manifold by

$$
L_{g}:=\Delta_{g}+\alpha_{n} \operatorname{Scal}_{g},
$$

where $\alpha_{n}:=\frac{n-2}{4(n-1)}$. The conformal covariance of $L_{g}$ expresses as

$$
L_{\hat{g}}(\phi)=u^{-\left(2^{*}-1\right)} L_{g}(u \phi) \quad \forall \phi \in C^{2}(M),
$$

for all $\hat{g}=u^{2^{*}-2} g$ in the conformal class $[g]$ of $g$. We assume that the manifold has positive Yamabe invariant, which is equivalent to assuming the coercivity of $L_{g}$. We let $H_{1}^{2}(M)$ be the Sobolev space of all functions in $L^{2}(M)$ with gradient in $L^{2}(M)$ equipped with the scalar product

$$
\langle u, v\rangle_{L_{g}}:=\int_{M}\langle\nabla u, \nabla v\rangle_{g} d v_{g}+\alpha_{n} \int_{M} \operatorname{Scal}_{g} u v d v_{g}
$$

where $d v_{g}$ is the volume element of the manifold. We let $\|\cdot\|_{L_{g}}$ be the norm induced by $\langle\cdot, \cdot\rangle_{L_{g}}$. For any $u \in L^{q}(M)$, we denote the $L^{q}$-norm of $u$ by $\|u\|_{q}:=\left(\int_{M}|u|^{q} d v_{g}\right)^{1 / q}$. We define $\|u\|_{1,2}:=\left(\|\nabla u\|_{2}^{2}+\|u\|_{2}^{2}\right)^{1 / 2}$. By coercivity of $L_{g}$, we get that the norms $\|\cdot\|_{L_{g}}$ and $\|\cdot\|_{1,2}$ are equivalent.

We let $i_{g}$ be the injectivity radius of the manifold $(M, g)$. By compactness of $M$, we get that there exists a positive real number $r_{0}$ such that $r_{0}<i_{g}$. In case $(M, g)$ is locally conformally flat, there exists a family $\left(g_{\xi}\right)_{\xi \in M}$ of smooth conformal metrics to $g$ such that $g_{\xi}$ is flat in the geodesic ball $B_{\xi}\left(r_{0}\right)$. In case $(M, g)$ is not locally conformally flat, we fix $N>n$, and we provide ourselves with a family $\left(g_{\xi}\right)_{\xi \in M}$ of smooth conformal metrics to $g$ such that

$$
\left|\exp _{\xi}^{*} g_{\xi}\right|(y)=1+\mathrm{O}\left(|y|^{N}\right)
$$

uniformly with respect to $\xi \in M$ and $y \in T_{\xi} M,|y| \ll 1$, where $\left|\exp _{\xi}^{*} g_{\xi}\right|$ is the determinant of $g_{\xi}$ in the geodesic normal coordinates of $g_{\xi}$ at $\xi$. Such coordinates are said to be conformal normal coordinates of order $N$ on the manifold. Here and in the sequel, the exponential map $\exp _{\xi}$ is always intended with respect to the metric $g_{\xi}$. We refer to Lee-Parker [27] for a proof 
of the existence of conformal normal coordinates of any finite order, see also the later proofs by Cao [11] and Günther [22] of the existence of conformal normal coordinates which are volume preserving near a given point (with no remainder term in (2.4)). For any $\xi \in M$, we let $\Lambda_{\xi}$ be the smooth positive function in $M$ such that $g_{\xi}=\Lambda_{\xi}^{2^{*}-2} g$. In both cases (locally conformally flat or not), the metric $g_{\xi}$ can be chosen smooth with respect to $\xi$ and such that $\Lambda_{\xi}(\xi)=1$ and $\nabla \Lambda_{\xi}(\xi)=0$. We let $G_{g}$ and $G_{g_{\xi}}$ be the respective Green's functions of $L_{g}$ and $L_{g_{\xi}}$. Using the fact that $\Lambda_{\xi}(\xi)=1$, by (2.2), we find that

$$
G_{g}(\cdot, \xi)=\Lambda_{\xi}(\cdot) G_{g_{\xi}}(\cdot, \xi) .
$$

By compactness of $M$ and since $g_{\xi}$ is smooth with respect to $\xi$, decreasing if necessary the real number $r_{0}$, we may assume that $r_{0}<i_{g_{\xi}}$ for all $\xi \in M$, where $i_{g_{\xi}}$ is the injectivity radius of the manifold $\left(M, g_{\xi}\right)$. For $\varepsilon>0$ small and for any $t>0$, we define

$$
\delta_{\varepsilon}(t):= \begin{cases}e^{-\frac{t}{\varepsilon}} & \text { if } n=4 \\ t \varepsilon^{\frac{1}{n-4}} & \text { if } n=5 \text { or }(n \geq 6 \text { and }(M, g) \text { l.c.f. }) \\ t \ell^{-1}(\varepsilon) & \text { if } n=6 \text { and }(M, g) \text { non-l.c.f. } \\ t \sqrt{\varepsilon} & \text { if } n \geq 7 \text { and }(M, g) \text { non-l.c.f., }\end{cases}
$$

where $\ell:\left(0, e^{-1 / 2}\right) \rightarrow\left(0, e^{-1} / 2\right)$ is given by $\ell(\delta):=-\delta^{2} \ln \delta$, and $\delta_{\varepsilon}:=\delta_{\varepsilon}(1)$. For $\varepsilon>0$ small and for any $t>0, \xi \in M$, inspired by the approach of Lee-Parker [27], we define $W_{\varepsilon, t, \xi}$ in $M$ by

with

$$
W_{\varepsilon, t, \xi}(x)=G_{g}(x, \xi) \widehat{W}_{\varepsilon, t, \xi}(x),
$$

$$
\widehat{W}_{\varepsilon, t, \xi}(x):= \begin{cases}\beta_{n} \delta_{\varepsilon}(t)^{\frac{2-n}{2}} d_{g_{\xi}}(x, \xi)^{n-2} U\left(\delta_{\varepsilon}(t)^{-1} d_{g_{\xi}}(x, \xi)\right) & \text { if } d_{g_{\xi}}(x, \xi) \leq r_{0} \\ \beta_{n} \delta_{\varepsilon}(t)^{\frac{2-n}{2}} r_{0}^{n-2} U\left(\delta_{\varepsilon}(t)^{-1} r_{0}\right) & \text { if } d_{g_{\xi}}(x, \xi)>r_{0},\end{cases}
$$

where $\beta_{n}=(n-2) \omega_{n-1}, \omega_{n-1}$ is the volume of the unit $(n-1)$-sphere, $\delta_{\varepsilon}(t)$ is as in (2.6) , and

$$
U(r):=\left(\frac{\sqrt{n(n-2)}}{1+r^{2}}\right)^{\frac{n-2}{2}} .
$$

The function $U$ generates a family $U_{\delta}(r)=\delta^{\frac{2-n}{2}} U\left(\delta^{-1} r\right), \delta>0$, of radial solutions of the equation $\Delta_{\text {Eucl }} U=U^{2^{*}-1}$ in $\mathbb{R}^{n}$, where $\Delta_{\text {Eucl }}:=-\operatorname{div}_{\text {Eucl }} \nabla$ is the Laplace operator with respect to the Euclidean metric. With these definitions, by (2.5), we observe that $W_{\varepsilon, t, \xi}$ rewrites as

$$
W_{\varepsilon, t, \xi}=\Lambda_{\xi}\left[\beta_{n} G_{g_{\xi}}(x, \xi) d_{g_{\xi}}(x, \xi)^{n-2}\right] U_{\delta_{\varepsilon}(t)}\left(d_{g_{\xi}}(x, \xi)\right)
$$

for all $x \in M$ such that $d_{g_{\xi}}(x, \xi) \leq r_{0}$.

Let us spend few words to comment on the choice of $W_{\varepsilon, t, \xi}$. Since, by Lemma A.1, the function $\beta_{n} G_{g_{\xi}}(x, \xi) d_{g_{\xi}}(x, \xi)^{n-2}$ is very close to 1 as $x \rightarrow \xi$, we have that $W_{\varepsilon, t, \xi}$ is a small correction of $\Lambda_{\xi} U_{\delta_{\varepsilon}(t)}\left(d_{g_{\xi}}(x, \xi)\right)$ near $\xi$. Since, by (2.2), we have that

$$
L_{g}\left(\Lambda_{\xi} U_{\delta_{\varepsilon}(t)}\left(d_{g_{\xi}}(x, \xi)\right)\right)=\Lambda_{\xi}^{2^{*}-1} L_{g_{\xi}}\left(U_{\delta_{\varepsilon}(t)}\left(d_{g_{\xi}}(x, \xi)\right)\right)
$$

in view of the flatness of $g_{\xi}$ at $\xi$ (see (2.4) ) and $\Delta_{\text {Eucl }} U_{\delta}=U_{\delta}^{2^{*}-1}$ in $\mathbb{R}^{n}$ it is natural to expect that $W_{\varepsilon, t, \xi}$ is a very good approximating solution to (1.3) near $\xi$. Away from $\xi$, the function $W_{\varepsilon, t, \xi}$ behaves like $\beta_{n}[n(n-2)]^{\frac{n-2}{4}} \delta_{\varepsilon}(t)^{\frac{n-2}{2}} G_{g}(x, \xi)$, which is still a good approximating solution to (1.3) in that region. 
We define $V_{0}, \ldots, V_{n}: \mathbb{R}^{n} \rightarrow \mathbb{R}$ by

$$
V_{0}(y):=\frac{|y|^{2}-1}{\left(1+|y|^{2}\right)^{\frac{n}{2}}} \quad \text { and } \quad V_{i}(y):=\frac{y_{i}}{\left(1+|y|^{2}\right)^{\frac{n}{2}}}
$$

for all $y \in \mathbb{R}^{n}$ and $i=1, \ldots, n$. By Bianchi-Egnell [6], any solution $v \in D^{1,2}\left(\mathbb{R}^{n}\right)$ to the equation $\Delta_{\text {Eucl }} v=\left(2^{*}-1\right) U^{2^{*}-2} v$ is a linear combination of the functions $V_{0}, \ldots, V_{n}$. We let $\chi$ be a smooth cutoff function in $\mathbb{R}_{+}$such that $0 \leq \chi \leq 1$ in $\mathbb{R}_{+}, \chi=1$ in $\left[0, r_{0} / 2\right]$, and $\chi=0$ in $\left[r_{0}, \infty\right)$. For $\varepsilon>0$ small and for any $t>0, \xi \in M$, and $\omega \in T_{\xi} M$, we define $Z_{\varepsilon, t, \xi}, Z_{\varepsilon, t, \xi, \omega}: M \rightarrow \mathbb{R}$ by

$$
Z_{\varepsilon, t, \xi}(x):=G_{g}(x, \xi) \widehat{Z}_{\varepsilon, t, \xi}(x) \quad \text { and } \quad Z_{\varepsilon, t, \xi, \omega}(x):=G_{g}(x, \xi) \widehat{Z}_{\varepsilon, t, \xi, \omega}(x),
$$

where

$$
\begin{aligned}
\widehat{Z}_{\varepsilon, t, \xi}(x) & :=\beta_{n} \chi\left(d_{g_{\xi}}(x, \xi)\right) \delta_{\varepsilon}(t)^{\frac{2-n}{2}} d_{g_{\xi}}(x, \xi)^{n-2} V_{0}\left(\delta_{\varepsilon}(t)^{-1} \exp _{\xi}^{-1} x\right), \\
\widehat{Z}_{\varepsilon, t, \xi, \omega}(x) & :=\beta_{n} \chi\left(d_{g_{\xi}}(x, \xi)\right) \delta_{\varepsilon}(t)^{\frac{2-n}{2}} d_{g_{\xi}}(x, \xi)^{n-2}\left\langle V\left(\delta_{\varepsilon}(t)^{-1} \exp _{\xi}^{-1} x\right), \omega\right\rangle_{g}
\end{aligned}
$$

with $V(y)=\left(V_{1}(y), \ldots, V_{n}(y)\right)$ for all $y \in T_{\xi} M \cong \mathbb{R}^{n}$. We define

$$
\begin{aligned}
& K_{\varepsilon, t, \xi}:=\left\{\lambda Z_{\varepsilon, t, \xi}+Z_{\varepsilon, t, \xi, \omega}: \quad \lambda \in \mathbb{R} \text { and } \omega \in T_{\xi} M\right\}, \\
& K_{\varepsilon, t, \xi}^{\perp}:=\left\{\phi \in H_{1}^{2}(M):\left\langle\phi, Z_{\varepsilon, t, \xi}\right\rangle_{L_{g}}=0 \text { and }\left\langle\phi, Z_{\varepsilon, t, \xi, \omega}\right\rangle_{L_{g}}=0 \text { for all } \omega \in T_{\xi} M\right\},
\end{aligned}
$$

where the scalar product $\langle\cdot, \cdot\rangle_{L_{g}}$ is as in (2.3). We let $\Pi_{\varepsilon, t, \xi}$ and $\Pi_{\varepsilon, t, \xi}^{\perp}$ be the respective projections of $H_{1}^{2}(M)$ onto $K_{\varepsilon, t, \xi}$ and $K_{\varepsilon, t, \xi}^{\perp}$.

We intend to construct solutions to equation (1.3) of the form

$$
u_{\varepsilon}:=W_{\varepsilon, t, \xi}+\phi_{\varepsilon},
$$

where $t>0, \xi \in M, \phi_{\varepsilon} \in K_{\varepsilon, t, \xi}^{\perp}$, and $W_{\varepsilon, t, \xi}$ is as in (2.7). We re-write equation (1.3) as the couple of equations

$$
\begin{aligned}
& \Pi_{\varepsilon, t, \xi}\left(W_{\varepsilon, t, \xi}+\phi-L_{g}^{-1}\left(f_{\varepsilon}\left(W_{\varepsilon, t, \xi}+\phi\right)\right)\right)=0, \\
& \Pi_{\varepsilon, t, \xi}^{\perp}\left(W_{\varepsilon, t, \xi}+\phi-L_{g}^{-1}\left(f_{\varepsilon}\left(W_{\varepsilon, t, \xi}+\phi\right)\right)\right)=0,
\end{aligned}
$$

where $L_{g}$ is as in (2.1) and

$$
f_{\varepsilon}(u):=u_{+}^{2^{*}-1}-\varepsilon h u,
$$

with $u_{+}=\max (u, 0)$. We begin with solving equation 2.17) in Proposition 2.1, a rather standard result in this context (see for instance Musso-Pistoia [35]) which proof is skipped for shortness.

Proposition 2.1. Given two positive real numbers $a<b$, there exists a positive constant $C=C(a, b, n, M, g, h)$ such that for $\varepsilon>0$ small, for any $t \in[a, b]$ and $\xi \in M$, there exists a unique function $\phi_{\varepsilon, t, \xi} \in K_{\varepsilon, t, \xi}^{\perp}$ which solves equation (2.17) and satisfies

$$
\left\|\phi_{\varepsilon, t, \xi}\right\|_{1,2} \leq C\left\|R_{\varepsilon, t, \xi}\right\|_{1,2},
$$

where $R_{\varepsilon, t, \xi}:=W_{\varepsilon, t, \xi}-L_{g}^{-1}\left(f_{\varepsilon}\left(W_{\varepsilon, t, \xi}\right)\right)$. Moreover, $\phi_{\varepsilon, t, \xi}$ is continuously differentiable with respect to $t$ and $\xi$.

In Proposition 2.2 below, we give a crucial estimate for $\left\|R_{\varepsilon, t, \xi}\right\|_{1,2}$. The proof of Proposition 2.2 is presented in Section 3 , 
Proposition 2.2. Given two positive real numbers $a<b$, there exists a positive constant $C=C(a, b, n, M, g, h)$ such that for $\varepsilon>0$ small, for any $t \in[a, b]$ and $\xi \in M$, there holds

$$
\left\|R_{\varepsilon, t, \xi}\right\|_{1,2} \leq C \begin{cases}\varepsilon e^{-\frac{t}{\varepsilon}} & \text { if } n=4 \\ \varepsilon^{\frac{5}{2}} & \text { if } n=5 \\ \varepsilon^{2}|\ln \varepsilon|^{\frac{2}{3}} & \text { if } n=6 \text { and }(M, g) \text { l.c.f. } \\ \varepsilon^{\frac{n+2}{2(n-4)}} & \text { if } n \geq 7 \text { and }(M, g) \text { l.c.f. } \\ \varepsilon^{2}|\ln \varepsilon|^{-\frac{1}{3}} & \text { if } n=6 \text { and }(M, g) \text { non-l.c.f. } \\ \varepsilon^{2} & \text { if } n \geq 7 \text { and }(M, g) \text { non-l.c.f. }\end{cases}
$$

where $R_{\varepsilon, t, \xi}$ is as in Proposition 2.1.

For $\varepsilon>0$ small, we define $J_{\varepsilon}: H_{1}^{2}(M) \rightarrow \mathbb{R}$ by

$$
J_{\varepsilon}(u):=\frac{1}{2} \int_{M}|\nabla u|_{g}^{2} d v_{g}+\frac{1}{2} \int_{M}\left(\alpha_{n} \operatorname{Scal}_{g}+\varepsilon h\right) u^{2} d v_{g}-\frac{1}{2^{*}} \int_{M}|u|^{2^{*}} d v_{g} .
$$

For any $t>0$ and $\xi \in M$ we define

$$
\mathcal{J}_{\varepsilon}(t, \xi):=J_{\varepsilon}\left(W_{\varepsilon, t, \xi}+\phi_{\varepsilon, t, \xi}\right)
$$

where $\phi_{\varepsilon, t, \xi}$ is given by Proposition 2.1. We can solve equation (2.16) by searching critical points of $\mathcal{J}_{\varepsilon}$, as it follows from (6.39)-(6.40) and (6.52) - To.53). This aim, it becomes crucial to have the asymptotic expansion of $\mathcal{J}_{\varepsilon}$ given by Proposition 2.3 below. The proof of Proposition 2.3 strongly relies on Propositions 2.1 and 2.2. , and is presented in section 4 .

We define the "reduced energy" $\widetilde{E}:(0, \infty) \times M \rightarrow \mathbb{R}$ as follows:

$$
\widetilde{E}(t, \xi)= \begin{cases}e^{-\frac{2 t}{\varepsilon}}\left(c_{2} t h(\xi)-c_{3} A_{\xi}\right) & \text { if } n=4 \\ c_{2} t^{2} h(\xi)-c_{3} t^{n-2} A_{\xi} & \text { if } n=5 \text { or }(n \geq 6 \text { and }(M, g) \text { l.c.f. }) \\ c_{2} t^{2} h(\xi)-c_{3} t^{4}\left|\operatorname{Weyl}_{g}(\xi)\right|_{g}^{2} & \text { if } n \geq 6 \text { and }(M, g) \text { non-l.c.f., }\end{cases}
$$

where $c_{2}, c_{3}>0$, Weyl $g$ is the Weyl curvature tensor of the manifold and $A_{\xi}$ is as in (1.5).

Proposition 2.3. Let $p \in\{0,1\}$ and assume that $h \in C^{0, \alpha}(M), \alpha \in(0,1)$, in case $p=0$ and $h \in C^{1}(M)$ in case $p=1$. Then there holds

$$
\mathcal{J}_{\varepsilon}(t, \xi)=c_{1}+ \begin{cases}\widetilde{E}(t, \xi)+\mathrm{o}\left(e^{-\frac{2 \varepsilon}{t}}\right) & \text { if } n=4 \\ \varepsilon^{\frac{n-2}{n-4}} \widetilde{E}(t, \xi)+\mathrm{o}\left(\varepsilon^{\frac{n-2}{n-4}}\right) & \text { if } n=5 \text { or }(n \geq 6 \text { and }(M, g) \text { l.c.f. }) \\ \varepsilon^{2}\left(\ln \frac{1}{\varepsilon}\right)^{-1} \widetilde{E}(t, \xi)+\mathrm{o}\left(\varepsilon^{2}\left(\ln \frac{1}{\varepsilon}\right)^{-1}\right) & \text { if } n=6 \text { and }(M, g) \text { non-l.c.f. } \\ \varepsilon^{2} \widetilde{E}(t, \xi)+\mathrm{o}\left(\varepsilon^{2}\right) & \text { if } n \geq 7 \text { and }(M, g) \text { non-l.c.f. }\end{cases}
$$

as $\varepsilon \rightarrow 0, C^{p}$-uniformly with respect to $\xi \in M$ and $t$ in compact subsets of $(0, \infty)$, where $\widetilde{E}$ is given by (2.23) and $c_{1}, c_{2}, c_{3}>0$ depend only on $n$.

We are now ready to state the following general result.

Theorem 2.4. Let $(M, g) \neq\left(\mathbb{S}^{n}, g_{0}\right)$ be a smooth compact Riemannian manifold with $n \geq 4$ and $\mu_{g}(M)>0$. Let $p \in\{0,1\}$ and assume that $h \in C^{0, \alpha}(M), \alpha \in(0,1)$, in case $p=0$ and $h \in C^{1}(M)$ in case $p=1$. Assume that there exists a $C^{p}$-stable critical set $\widetilde{\mathcal{D}} \subset(0, \infty) \times M$, $p=0,1$, of the function $\widetilde{E}$. Then for $\varepsilon>0$ small, equation (1.3) has a solution $u_{\varepsilon} \in C^{2, \alpha}(M)$, $\forall \alpha \in(0,1)$ if $p=1$, such that the family $\left(u_{\varepsilon}\right)_{\varepsilon}$ blows up, up to a sub-sequence, at some $\xi_{0} \in \pi(\widetilde{\mathcal{D}})$ as $\varepsilon \rightarrow+\infty$. Here, $\pi:(0, \infty) \times M \rightarrow M$ is the projection operator onto the second 
component. Moreover, when $p=1, \xi_{0}$ is a critical point of $E$ with $h\left(\xi_{0}\right)>0$, where $E$ is defined in (1.4).

The notion of stability we are using is essentially taken from Li [29]. We say that a compact set $\widetilde{\mathcal{D}} \subset(0, \infty) \times M$ is a $C^{p}$-stable critical set, $p \in \mathbb{N}$, if for any compact neighborhood $\widetilde{U}$ of $\widetilde{\mathcal{D}}$ in $(0, \infty) \times M$, there exists $\delta>0$ such that, if $\widetilde{J} \in C^{1}(\widetilde{U})$ and $\|\widetilde{\mathcal{J}}-\widetilde{E}\|_{C^{p}(\widetilde{U})} \leq \delta$, then $\widetilde{\mathcal{J}}$ has at least one critical point in $\widetilde{U}$. Since $\widetilde{E}$ depends on $\varepsilon$ when $n=4$, the above assumption $\|\widetilde{\mathcal{J}}-\widetilde{E}\|_{C^{p}(\widetilde{U})} \leq \delta$ needs to be interpreted in this case as: $|\widetilde{\mathcal{J}}(t, \xi)-\widetilde{E}(t, \xi)| \leq \delta e^{-\frac{2 t}{\varepsilon}}$ for all $(t, \xi) \in \widetilde{U}$, when $p=0 ;|\widetilde{\mathcal{J}}(t, \xi)-\widetilde{E}(t, \xi)|+\varepsilon\left|\partial_{t}(\tilde{\mathcal{J}}(t, \xi)-\widetilde{E}(t, \xi))\right|+\left|\nabla_{\xi}(\tilde{\mathcal{J}}(t, \xi)-\widetilde{E}(t, \xi))\right|_{g} \leq$ $\delta e^{-\frac{2 t}{\varepsilon}}$ for all $(t, \xi) \in \widetilde{U}$, when $p=1$. Observe also that we do not require the set $\widetilde{\mathcal{D}}$ to be composed of critical points of $\widetilde{E}$, as it would be intuitively reasonable. Indeed, we want to include the case where $\widetilde{\mathcal{D}}$ is given by almost critical points of $\widetilde{E}$, as it arises for $n=4$ in the proof of Theorem 1.1. However, since $\widetilde{U}$ can shrink onto $\widetilde{\mathcal{D}}$, by compactness of $\widetilde{\mathcal{D}}$, we have that $\widetilde{\mathcal{D}}$ contains at least one critical point of $\widetilde{E}$.

Proof of Theorem 2.4. Let $\widetilde{U}$ be a compact neighborhood of the $C^{p}$-stable critical set $\widetilde{\mathcal{D}}$ in $(0, \infty) \times M, p=0,1$. Given any $\delta>0$, by Proposition 2.3, we have that $\widetilde{\mathcal{J}}_{\varepsilon}:=\mu_{\varepsilon}^{-1}\left(\mathcal{J}_{\varepsilon}-c_{1}\right)$ satisfies $\left\|\widetilde{\mathcal{J}}_{\varepsilon}-\widetilde{E}\right\|_{C^{p}(\widetilde{U})} \leq \delta$ for $\varepsilon$ sufficiently small, where $\mu_{\varepsilon}=1$ if $n=4, \mu_{\varepsilon}:=\varepsilon^{\frac{n-2}{n-4}}$ if $n=5$ or $n \geq 6$ and $(M, g)$ l.c.f., $\mu_{\varepsilon}:=\varepsilon^{2}\left(\ln \frac{1}{\varepsilon}\right)^{-1}$ if $n=6$ and $(M, g)$ non-l.c.f., $\mu_{\varepsilon}:=\varepsilon^{2}$ if $n \geq 7$ and $(M, g)$ non-l.c.f. By definition of a $C^{p}$-stable critical set, $p=0,1$, it follows that $\mathcal{J}_{\varepsilon}$ has a critical point $\left(t_{\varepsilon}, \xi_{\varepsilon}\right) \in \widetilde{U}$ for $\varepsilon$ small. Up to a subsequence and taking $\widetilde{U}$ smaller and smaller, we can assume that $\left(t_{\varepsilon}, \xi_{\varepsilon}\right) \rightarrow\left(t_{0}, \xi_{0}\right)$ as $\varepsilon \rightarrow 0$ with $\xi_{0} \in \pi(\widetilde{\mathcal{D}})$. As already observed, we get that $u_{\varepsilon}=W_{\varepsilon, t_{\varepsilon}, \xi_{\varepsilon}}+\phi_{\varepsilon, t_{\varepsilon}, \xi_{\varepsilon}}$ is a critical point of $J_{\varepsilon}$, and thus, by elliptic regularity, a classical solution of (1.3). Since $\left\|\phi_{\varepsilon, t_{\varepsilon}, \xi_{\varepsilon}}\right\|_{1,2} \rightarrow 0$, by definition of $W_{\varepsilon, t_{\varepsilon}, \xi_{\varepsilon}}$, it is easily seen that $u_{\varepsilon}>0$ and $u_{\varepsilon}^{2^{*}} \rightarrow K_{n}^{-n} \delta_{\xi_{0}}$ in the measures sense as $\varepsilon \rightarrow 0$ (see for instance Rey [40]), where $K_{n}$ is given by (4.1) and $\delta_{\xi_{0}}$ denotes the Dirac mass measure at $\xi_{0}$. From very basic facts concerning the asymptotic analysis of solutions of Yamabe-type equations (see for instance Druet-Hebey [16] and Druet-Hebey-Robert [18]) we get that the family $\left(u_{\varepsilon}\right)_{\varepsilon}$ of solutions to (1.3) blows up at the point $\xi_{0}$ as $\varepsilon \rightarrow 0$. Finally, when $p=1$, we can pass to the limit into the equations $\partial_{t} \mathcal{J}_{\varepsilon}\left(t_{\varepsilon}, \xi_{\varepsilon}\right)=0$ and $\nabla_{\xi} \mathcal{J}_{\varepsilon}\left(t_{\varepsilon}, \xi_{\varepsilon}\right)=0$ as $\varepsilon \rightarrow 0$ to get that $h\left(\xi_{0}\right)>0$ and $\nabla_{\xi} E\left(\xi_{0}\right)=0$ in view of $t_{0}>0$, where $E$ is given by (1.4). This ends the proof of Theorem 2.4.

We now apply Theorem 2.4 to deduce Theorems 1.1 and 1.2 . To this aim, given $\xi \in M$ with $h(\xi)>0$, define $t(\xi)$ as

$$
t(\xi):= \begin{cases}\frac{c_{3} A_{\xi}}{c_{2} h(\xi)}+\frac{\varepsilon}{2} & \text { if } n=4 \\ \left(\frac{2 c_{2} h(\xi)}{(n-2) c_{3} A_{\xi}}\right)^{\frac{1}{n-4}} & \text { if } n=5 \text { or }(n \geq 6 \text { and }(M, g) \text { l.c.f. }) \\ \left(\frac{c_{2} h(\xi)}{2 c_{3}\left|\operatorname{Weyl}_{g}(\xi)\right|_{g}^{2}}\right)^{\frac{1}{2}} & \text { if } n \geq 6 \text { and }(M, g) \text { non-l.c.f. }\end{cases}
$$

with the convention that $1 / 0=+\infty$. One easily checks that every $t(\xi)<+\infty$ is a global maximum point of $\widetilde{E}$ in $t$. In the proofs of Theorems 1.1 and 1.2 below, we show that the $C^{p}$-stable critical set $\widetilde{\mathcal{D}}$ in Theorem 2.4 can be constructed as $\widetilde{\mathcal{D}}:=\{(t(\xi), \xi): \xi \in \mathcal{D}\}$, where 


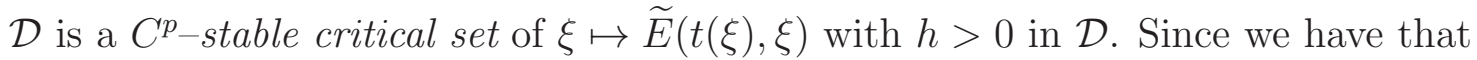

$$
\widetilde{E}(t(\xi), \xi)= \begin{cases}\frac{\varepsilon}{2} c_{2} e^{-1} h(\xi) e^{-\frac{2 c_{3}}{\varepsilon c_{2}} \cdot \frac{A_{\xi}}{h(\xi)}} & \text { if } n=4 \\ \frac{2^{\frac{2}{n-4}}(n-4) c_{2}^{\frac{n-2}{n-4}}}{(n-2)^{\frac{n-2}{n-4}} c_{3}^{\frac{2}{n-4}}} \cdot \frac{h(\xi)^{\frac{n-2}{n-4}}}{A_{\xi}^{\frac{2}{n-4}}} & \text { if } n=5 \text { or }(n \geq 6 \text { and }(M, g) \text { l.c.f. }) \\ \frac{c_{2}^{2}}{4 c_{3}} \cdot \frac{h(\xi)^{2}}{\mid \text { Weyl }\left._{g}(\xi)\right|_{g} ^{2}} & \text { if } n \geq 6 \text { and }(M, g) \text { non-l.c.f., }\end{cases}
$$

the role played by the map $E$ defined in (1.4) becomes clear. We prove Theorem 1.1 as follows.

Proof of Theorem 1.1. Notice that $\sup _{M} E>0$ in view of $\max _{M} h>0$. Letting $\left(\xi_{k}\right)_{k \in \mathbb{N}}$ be a maximizing sequence for $E$, by compactness of $M$, we can assume that $\xi_{k} \rightarrow \xi$ as $k \rightarrow+\infty$ with $h\left(\xi_{k}\right)>0$ for all $k$. Since $\min \left\{\left|\operatorname{Weyl}_{g}(\xi)\right|_{g}: h(\xi)>0\right\}>0$ in the non-l.c.f. case with $n \geq 6$, we have that $\left(E\left(\xi_{k}\right)\right)_{k}$ is bounded. Since then $\sup _{M} E<+\infty$, it follows that $\sup _{M} E$ is achieved, and it makes sense to define $\mathcal{D}:=\left\{\xi \in M: E(\xi)=\sup _{M} E\right\}$ (possibly coinciding with the whole $M$ if $E$ is a constant function) as the maximal set of $E$ in $M$. Correspondingly, define $\widetilde{\mathcal{D}}:=\{(t(\xi), \xi): \xi \in \mathcal{D}\}$, where $t(\xi)$ is given by (2.24) and is well defined for all $\xi \in \mathcal{D}$ in view of $h>0$ in $\mathcal{D}$. Moreover, $t(\xi)$ is clearly bounded away from zero on $\mathcal{D}$, and thus the set $\widetilde{\mathcal{D}}$ is a compact set in $(0, \infty) \times M$. To show that $\widetilde{\mathcal{D}}$ is a $C^{0}$-stable critical set, let $\widetilde{U}$ be a compact neighborhood of $\widetilde{\mathcal{D}}$ in $(0, \infty) \times M$. Taking $\widetilde{U}$ smaller if necessary, we can assume that $\widetilde{U}=\{(t, \xi): t \in[t(\xi)-\eta, t(\xi)+\eta], \xi \in U\}$, where $\eta>0$ is small and $U$ is a closed neighborhood of $\mathcal{D}$ in $M$ so that $h>0$ in $U$. There hold

- for $n \geq 5$, by the definition of $t(\xi)$ and the simple relation between $\widetilde{E}(t(\xi), \xi)$ and $E(\xi)$ (see (1.4) and (2.25)), we have that $\widetilde{E}(t(\xi) \pm \eta, \xi)<\widetilde{E}(t(\xi), \xi) \leq \sup _{\widetilde{\mathcal{D}}} \widetilde{E}$ if $\xi \in U$, and $\widetilde{E}(t, \xi) \leq \widetilde{E}(t(\xi), \xi)<\sup _{\widetilde{\mathcal{D}}} \widetilde{E}$ if $t \in[t(\xi)-\eta, t(\xi)+\eta]$ and $\xi \in \partial U$

- for $n=4$, by the definition of $\widetilde{E}$ and $t(\xi)$, we have that for any $\xi \in U$, there holds

$$
\widetilde{E}(t(\xi) \pm \eta, \xi)=\widetilde{E}(t(\xi), \xi) e^{\mp \frac{2 \eta}{\varepsilon}}\left(1 \pm \frac{2 \eta}{\varepsilon}\right)<\sup _{\widetilde{\mathcal{D}}} \widetilde{E}
$$

when $\varepsilon$ is small, in view of $e^{-\frac{2 \eta}{\varepsilon}}\left(1+\frac{2 \eta}{\varepsilon}\right) \rightarrow 0$ and $e^{\frac{2 \eta}{\varepsilon}}\left(1-\frac{2 \eta}{\varepsilon}\right) \rightarrow-\infty$ as $\varepsilon \rightarrow 0$, and for any $t \in[t(\xi)-\eta, t(\xi)+\eta]$ and $\xi \in \partial U$, there holds

$$
\widetilde{E}(t, \xi) \leq \widetilde{E}(t(\xi), \xi)<\sup _{\widetilde{\mathcal{D}}} \widetilde{E}
$$

when $\varepsilon$ is small, in view of $\sup _{\partial U} e^{-\frac{2 c_{3}}{\varepsilon c_{2}} \frac{A \xi}{h(\xi)}}=\mathrm{o}\left(e^{-\frac{2 c_{3}}{\varepsilon c_{2} \max _{M} E}}\right)$ as $\varepsilon \rightarrow 0$.

In conclusion, by compactness of

$$
\partial \widetilde{U}=\{(t, \xi): t \in\{t(\xi)-\eta, t(\xi)+\eta\}, \xi \in U\} \cup\{(t, \xi): t \in[t(\xi)-\eta, t(\xi)+\eta], \xi \in \partial U\},
$$

we get that $\sup _{\partial \widetilde{U}} \widetilde{E}<\sup _{\widetilde{\mathcal{D}}} \widetilde{E}$. It follows that if $\|\widetilde{\mathcal{J}}-\widetilde{E}\|_{C^{0}(\widetilde{U})} \leq \delta$, with $\delta<\frac{1}{2}\left[\sup _{\widetilde{\mathcal{D}}} \widetilde{E}-\right.$ $\left.\sup _{\partial \widetilde{U}} \widetilde{E}\right]$, then we get that

$$
\sup _{\partial \widetilde{U}} \tilde{\mathcal{J}}<\sup _{\widetilde{\mathcal{D}}} \tilde{\mathcal{J}}
$$

Then $\widetilde{\mathcal{J}}$ achieves its maximum value in $\widetilde{U}$ at some interior point of $\widetilde{U}$, which is a critical point of $\widetilde{\mathcal{J}}$. It follows that $\widetilde{\mathcal{D}}$ is a $C^{0}$-stable critical set of $\widetilde{E}$ as desired. By Theorem 2.4, we then get that for $\varepsilon>0$ small, equation (1.3) has a solution $u_{\varepsilon} \in C^{2, \alpha}(M)$ such that the family 
$\left(u_{\varepsilon}\right)_{\varepsilon}$ blows up, up to a sub-sequence, at some $\xi_{0} \in \pi(\widetilde{\mathcal{D}})$ as $\varepsilon \rightarrow+\infty$. Moreover, by definition of $\widetilde{\mathcal{D}}$, we get that $\widetilde{E}\left(\xi_{0}\right)=\max _{M} \widetilde{E}$. This ends the proof of Theorem 1.1 .

Since $t(\xi)$ is a maximum point of $\widetilde{E}$ in $t$, minimum points or saddle points of $E$ provide critical points of $\widetilde{E}$ which in general are not $C^{0}$-stable critical points of $\widetilde{E}$. To cover these cases, we need to use Theorem 2.4 with $p=1$. We assume that $h \in C^{1}(M)$. We can still define $C^{1}$-stable critical sets of $E$ as in the case of $(0, \infty) \times M$, but in general they don't give rise to $C^{1}$-stable critical sets of $\widetilde{E}$. In $M$, we restrict the notion of $C^{1}$-stability to isolated critical points of $E$ with non-trivial local degree, which is still sufficiently general to include non-degenerate critical points of $E$. We prove Theorem 1.2 as follows.

Proof of Theorem 1.2. We only need to show that the set $\widetilde{\mathcal{D}}:=\left\{\left(t\left(\xi_{0}\right), \xi_{0}\right)\right\}$ is a $C^{1}-$ stable critical set of $\widetilde{E}$, where $t\left(\xi_{0}\right)>0$ is well defined in view of $h\left(\xi_{0}\right)>0$ and $\operatorname{Weyl}_{g}\left(\xi_{0}\right) \neq 0$ when $n \geq 6$ and $(M, g)$ non-l.c.f. To this aim, let $\widetilde{U}$ be any compact neighborhood of $\widetilde{\mathcal{D}}$ in $(0, \infty) \times M$ and $\delta>0$ be any given small number. Taking $\widetilde{U}$ smaller if necessary, we can assume that $\widetilde{U}=I \times B_{\xi_{0}}\left(r_{0}\right)$, where $I$ is a closed interval in $(0, \infty)$ containing $t\left(\xi_{0}\right)$ in its interior and $B_{\xi_{0}}\left(r_{0}\right)$ is the geodesic ball of center $\xi_{0}$ and radius $r_{0}$ with respect to $g_{\xi_{0}}$, with $r_{0}<i_{g_{\xi_{0}}}$, the injectivity radius of the manifold $\left(M, g_{\xi_{0}}\right)$. For $n \geq 5$, the assumption $\|\widetilde{\mathcal{J}}-\widetilde{E}\|_{C^{1}(\widetilde{U})} \leq \delta$ gives that

$$
\left|\nabla \tilde{\mathcal{J}}\left(t, \exp _{\xi_{0}} \eta\right)-\nabla \widetilde{E}\left(t, \exp _{\xi_{0}} \eta\right)\right| \leq \delta
$$

as $\varepsilon \rightarrow 0$, uniformly with respect to $\eta \in B_{0}\left(r_{0}\right)$ and $t \in I$, where $\nabla:=\left(d / d t, \nabla_{\eta}\right)$. Since $\xi_{0}$ is a $C^{1}$-stable critical point of $E$ with $h\left(\xi_{0}\right)>0$, where $E$ is given by (1.4), we find that $\nabla \widetilde{E}\left(t, \exp _{\xi_{0}} \eta\right)$ has an isolated zero at $\left(t\left(\xi_{0}\right), 0\right)$. Since $t\left(\xi_{0}\right)$ is a non-degenerate critical point of $t \mapsto \widetilde{E}\left(t, \exp _{\xi_{0}} \eta\right)$, taking $\widetilde{U}$ smaller if necessary, we also have that $\operatorname{deg}(\nabla \widetilde{E}, \widetilde{U}, 0) \neq 0$ (see for instance [21]). It follows from (2.26) that if $\delta$ is small enough, then $\widetilde{\mathcal{J}}$ has at least one critical point $(t, \xi) \in \widetilde{U}$. When $n=4$, the assumption $\|\widetilde{\mathcal{J}}-\widetilde{E}\|_{C^{1}(\widetilde{U})} \leq \delta$ gives (by definition) that

$$
\varepsilon e^{\frac{2 t}{\varepsilon}}\left|\partial_{t} \widetilde{\mathcal{J}}\left(t, \exp _{\xi_{0}} \eta\right)-\partial_{t} \widetilde{E}\left(t, \exp _{\xi_{0}} \eta\right)\right|+e^{\frac{2 t}{\varepsilon}}\left|\nabla_{\eta} \widetilde{\mathcal{J}}\left(t, \exp _{\xi_{0}} \eta\right)-\nabla_{\eta} \widetilde{E}\left(t, \exp _{\xi_{0}} \eta\right)\right| \leq C_{0} \delta
$$

as $\varepsilon \rightarrow 0$, uniformly with respect to $\eta \in B_{0}\left(r_{0}\right)$ and $t \in I$, for some $C_{0}>0$. Letting

$$
\Psi(t, \eta)=\left(-2 c_{2} t h\left(\exp _{\xi_{0}} \eta\right)+2 c_{3} A_{\exp _{\xi_{0}} \eta}, c_{2} t \nabla_{\eta} h\left(\exp _{\xi_{0}} \eta\right)-c_{3} \nabla_{\eta} A_{\exp _{\xi_{0}} \eta}\right)
$$

and $\widetilde{\Psi}(t, \xi)=\Psi\left(t, \exp _{\xi_{0}}^{-1} \xi\right)$, by (2.27) we deduce that

$$
\left|\left(\varepsilon e^{\frac{2 t}{\varepsilon}} \partial_{t} \widetilde{\mathcal{J}}\left(t, \exp _{\xi_{0}} \eta\right), e^{\frac{2 t}{\varepsilon}} \nabla_{\eta} \widetilde{\mathcal{J}}\left(t, \exp _{\xi_{0}} \eta\right)\right)-\Psi(t, \eta)\right| \leq C_{0} \delta+\mathrm{O}(\varepsilon)
$$

as $\varepsilon \rightarrow 0$, uniformly with respect to $\eta \in B_{0}\left(r_{0}\right)$ and $t \in I$. Arguing as above, the map $\Psi$ has an isolated zero at $\left(\frac{c_{3} A_{\xi_{0}}}{c_{2} h\left(\xi_{0}\right)}, 0\right)$ with $\operatorname{deg}(\widetilde{\Psi}, \widetilde{U}, 0) \neq 0$, and then by (2.28), it follows that if $\delta$ is small enough, then $\widetilde{\mathcal{J}}$ has at least one critical point $(t, \xi) \in \widetilde{U}$.

\section{The ERRor ESTIMATE}

This section is devoted to the estimate of $R_{\varepsilon, t, \xi}$. 
Proof of Proposition 2.2. All our estimates in this proof are uniform with respect to $t \in[a, b]$, $\xi \in M$ and $\varepsilon \in\left(0, \varepsilon_{0}\right)$, for some fixed $\varepsilon_{0}>0$. We let $r_{0}$ be as in Section 2. For any $\phi \in H_{1}^{2}(M)$, an integration by parts gives that

$$
\begin{aligned}
\left\langle L_{g}^{-1}\left(f_{\varepsilon}\left(W_{\varepsilon, t, \xi}\right)\right)-W_{\varepsilon, t, \xi}, \phi\right\rangle_{L_{g}} & =\int_{M}\left(f_{\varepsilon}\left(W_{\varepsilon, t, \xi}\right)-L_{g} W_{\varepsilon, t, \xi}\right) \phi d v_{g} \\
& -\int_{\partial B_{\xi}\left(r_{0}\right)}\left(\partial_{\nu_{\mathrm{in}}} W_{\varepsilon, t, \xi}+\partial_{\nu_{\mathrm{out}}} W_{\varepsilon, t, \xi}\right) \phi d \sigma_{g},
\end{aligned}
$$

where $\partial B_{\xi}\left(r_{0}\right)$ is the boundary of the geodesic ball with respect to $g_{\xi}$ of center $\xi$ and radius $r_{0}, \partial_{\nu_{\text {in }}}$ and $\partial_{\nu_{\text {out }}}$ are the derivatives with respect to the respective inward and outward, unit, normal vectors to $\partial B_{\xi}\left(r_{0}\right)$, and $d \sigma_{g}$ is the volume element on $\partial B_{\xi}\left(r_{0}\right)$. By Sobolev's and trace's embeddings, it follows from (3.1) that

$$
\begin{aligned}
\left\|L_{g}^{-1}\left(f_{\varepsilon}\left(W_{\varepsilon, t, \xi}\right)\right)-W_{\varepsilon, t, \xi}\right\|_{1,2}=\mathrm{O}\left(\left\|f_{\varepsilon}\left(W_{\varepsilon, t, \xi}\right)-L_{g} W_{\varepsilon, t, \xi}\right\|_{L^{\frac{2 n}{n+2}}(M)}\right. \\
\left.+\left\|\partial_{\nu_{\mathrm{in}}} W_{\varepsilon, t, \xi}+\partial_{\nu_{\mathrm{out}}} W_{\varepsilon, t, \xi}\right\|_{L^{\frac{2(n-1)}{n}}\left(\partial B_{\xi}\left(r_{0}\right)\right)}\right) .
\end{aligned}
$$

Regarding the second term in the right hand side of (3.2), on $\partial B_{\xi}\left(r_{0}\right)$, we find that

$$
\begin{aligned}
\partial_{\nu_{\mathrm{in}}} W_{\varepsilon, t, \xi}+\partial_{\nu_{\mathrm{out}}} W_{\varepsilon, t, \xi} & =\left.\beta_{n} \delta_{\varepsilon}(t)^{\frac{2-n}{2}} G_{g}(\cdot, \xi) \frac{d}{d r}\left(r^{n-2} U\left(\delta_{\varepsilon}(t)^{-1} r\right)\right)\right|_{r=r_{0}} \\
& =n^{\frac{n-2}{4}}(n-2)^{\frac{n+2}{4}} \beta_{n} G_{g}(\cdot, \xi) \frac{\delta_{\varepsilon}(t)^{\frac{n+2}{2}} r_{0}^{n-3}}{\left(\delta_{\varepsilon}(t)^{2}+r_{0}^{2}\right)^{\frac{n}{2}}}=\mathrm{O}\left(\delta_{\varepsilon}(t)^{\frac{n+2}{2}}\right) .
\end{aligned}
$$

Regarding the first term in the right hand side of (3.2), we observe that in $M \backslash B_{\xi}\left(r_{0}\right)$, there holds

$$
f_{\varepsilon}\left(W_{\varepsilon, t, \xi}\right)-L_{g} W_{\varepsilon, t, \xi}=f_{\varepsilon}\left(W_{\varepsilon, t, \xi}\right)=\mathrm{O}\left(\delta_{\varepsilon}(t)^{\frac{n+2}{2}}+\varepsilon \delta_{\varepsilon}(t)^{\frac{n-2}{2}}\right)
$$

in view of

$$
\widehat{W}_{\varepsilon, t, \xi}=\beta_{n} \delta_{\varepsilon}(t)^{\frac{2-n}{2}} r_{0}^{n-2} U\left(\delta_{\varepsilon}(t)^{-1} r_{0}\right)=\mathrm{O}\left(\delta_{\varepsilon}(t)^{\frac{n-2}{2}}\right)
$$

and $L_{g} G_{g}(\cdot, \xi)=0$. By conformal covariance (2.2) of $L_{g}$ and by (2.5), in $B_{\xi}\left(r_{0}\right)$, we can write that

$$
f_{\varepsilon}\left(W_{\varepsilon, t, \xi}\right)-L_{g} W_{\varepsilon, t, \xi}=\Lambda_{\xi}^{2^{*}-1}\left[\left(G_{g_{\xi}}(\cdot, \xi) \widehat{W}_{\varepsilon, t, \xi}\right)^{2^{*}-1}-L_{g_{\xi}}\left(G_{g_{\xi}}(\cdot, \xi) \widehat{W}_{\varepsilon, t, \xi}\right)\right]-\varepsilon h W_{\varepsilon, t, \xi} .
$$

Since $\widehat{W}_{\varepsilon, t, \xi}(\xi)=0$ and $L_{g_{\xi}} G_{g_{\xi}}(\cdot, \xi)=\delta_{\xi}$, we get that

$$
L_{g_{\xi}}\left(G_{g_{\xi}}(\cdot, \xi) \widehat{W}_{\varepsilon, t, \xi}\right)=G_{g_{\xi}}(\cdot, \xi) \Delta_{g_{\xi}} \widehat{W}_{\varepsilon, t, \xi}-2\left\langle\nabla G_{g_{\xi}}(\cdot, \xi), \nabla \widehat{W}_{\varepsilon, t, \xi}\right\rangle_{g_{\xi}} .
$$

Since $\widehat{W}_{\varepsilon, t, \xi} \circ \exp _{\xi}$ is radially symmetrical in $B_{0}\left(r_{0}\right)$, writing $\Delta_{g_{\xi}} \widehat{W}_{\varepsilon, t, \xi}\left(\exp _{\xi} y\right)$ in polar coordinates, by (2.4), we find that

$$
\begin{aligned}
\Delta_{g_{\xi}} \widehat{W}_{\varepsilon, t, \xi}\left(\exp _{\xi} y\right)= & \Delta_{\text {Eucl }}\left(\widehat{W}_{\varepsilon, t, \xi} \circ \exp _{\xi}\right)(y)+\mathrm{O}\left(|y|^{N-1}\left|\nabla\left(\widehat{W}_{\varepsilon, t, \xi} \circ \exp _{\xi}\right)(y)\right|\right) \\
= & \beta_{n} \delta_{\varepsilon}(t)^{-\frac{n+2}{2}}|y|^{n-2} U\left(\frac{y}{\delta_{\varepsilon}(t)}\right)^{2^{*}-1}-2 n^{\frac{n-2}{4}}(n-2)^{\frac{n+6}{4}} \beta_{n} \frac{\delta_{\varepsilon}(t)^{\frac{n+2}{2}}|y|^{n-4}}{\left(\delta_{\varepsilon}(t)^{2}+|y|^{2}\right)^{\frac{n}{2}}} \\
& +\mathrm{O}\left(\frac{\delta_{\varepsilon}(t)^{\frac{n+2}{2}}|y|^{N+n-4}}{\left(\delta_{\varepsilon}(t)^{2}+|y|^{2}\right)^{\frac{n}{2}}}\right)
\end{aligned}
$$


uniformly with respect to $y \in B_{0}\left(r_{0}\right)$, in view of $\Delta_{\text {Eucl }} U=U^{2^{*}-1}$ in $\mathbb{R}^{n}$. Moreover, since $\widehat{W}_{\varepsilon, t, \xi} \circ \exp _{\xi}$ is radially symmetrical, we get that

$$
\begin{array}{r}
\left\langle\nabla G_{g_{\xi}}\left(\exp _{\xi} y, \xi\right), \nabla \widehat{W}_{\varepsilon, t, \xi}\left(\exp _{\xi} y\right)\right\rangle_{g_{\xi}}=\partial_{r}\left[G_{g_{\xi}}\left(\exp _{\xi} y, \xi\right)\right] \partial_{r}\left[\widehat{W}_{\varepsilon, t, \xi} \circ \exp _{\xi}(y)\right] \\
=\partial_{r}\left[G_{g_{\xi}}\left(\exp _{\xi} y, \xi\right)\right] n^{\frac{n-2}{4}}(n-2)^{\frac{n+2}{4}} \beta_{n} \frac{\delta_{\varepsilon}(t)^{\frac{n+2}{2}}|y|^{n-3}}{\left(\delta_{\varepsilon}(t)^{2}+|y|^{2}\right)^{\frac{n}{2}}}
\end{array}
$$

for all $y \in B_{0}\left(r_{0}\right)$. Inserting (3.7) and (3.8) into (3.6), we get that

$$
\begin{aligned}
& L_{g_{\xi}}\left(G_{g_{\xi}}(\cdot, \xi) \widehat{W}_{\varepsilon, t, \xi}\right)\left(\exp _{\xi} y\right)=\beta_{n} G_{g_{\xi}}\left(\exp _{\xi} y, \xi\right)|y|^{n-2} \delta_{\varepsilon}(t)^{-\frac{n+2}{2}} U\left(\delta_{\varepsilon}(t)^{-1} y\right)^{2^{*}-1} \\
& -2 n^{\frac{n-2}{4}}(n-2)^{\frac{n+2}{4}} \beta_{n}\left((n-2) G_{g_{\xi}}\left(\exp _{\xi} y, \xi\right)+|y| \partial_{r}\left[G_{g_{\xi}}\left(\exp _{\xi} y, \xi\right)\right]\right) \frac{\delta_{\varepsilon}(t)^{\frac{n+2}{2}}|y|^{n-4}}{\left(\delta_{\varepsilon}(t)^{2}+|y|^{2}\right)^{\frac{n}{2}}} \\
& +\mathrm{O}\left(\frac{\delta_{\varepsilon}(t)^{\frac{n+2}{2}} G_{g_{\xi}}\left(\exp _{\xi} y, \xi\right)|y|^{N+n-4}}{\left(\delta_{\varepsilon}(t)^{2}+|y|^{2}\right)^{\frac{n}{2}}}\right)
\end{aligned}
$$

in $B_{0}\left(r_{0}\right)$. Using Lemma A.1, by (3.9) we find that

$$
\begin{aligned}
& \left(G_{g_{\xi}}\left(\exp _{\xi} y, \xi\right) \widehat{W}_{\varepsilon, t, \xi}\left(\exp _{\xi} y\right)\right)^{2^{*}-1}-L_{g_{\xi}}\left(G_{g_{\xi}}(\cdot, \xi) \widehat{W}_{\varepsilon, t, \xi}\right)\left(\exp _{\xi} y\right) \\
& =\frac{\delta_{\varepsilon}(t)^{\frac{n+2}{2}}}{\left(\delta_{\varepsilon}(t)^{2}+|y|^{2}\right)^{\frac{n}{2}}} \times \begin{cases}\mathrm{O}\left(|y|^{n-4}\right) & \text { if } n=4,5 \text { or }(M, g) \text { l.c.f. } \\
\mathrm{O}\left(|y|^{2} \ln |y|\right) & \text { if } n=6 \text { and }(M, g) \text { non-l.c.f. } \\
\mathrm{O}\left(|y|^{2}\right) & \text { if } n \geq 7 \text { and }(M, g) \text { non-l.c.f. }\end{cases}
\end{aligned}
$$

in $B_{0}\left(r_{0}\right)$. It follows from (3.10) that

$$
\begin{aligned}
\int_{B_{\xi}\left(r_{0}\right)} & \Lambda_{\xi}^{\frac{2 n}{n-2}}\left|\left(G_{g_{\xi}}(\cdot, \xi) \widehat{W}_{\varepsilon, t, \xi}\right)^{2^{*}-1}-L_{g_{\xi}}\left(G_{g_{\xi}}(\cdot, \xi) \widehat{W}_{\varepsilon, t, \xi}\right)\right|^{\frac{2 n}{n+2}} d v_{g} \\
= & \int_{0}^{r_{0}} \frac{\delta_{\varepsilon}(t)^{n} d r}{\left(\delta_{\varepsilon}(t)^{2}+r^{2}\right)^{\frac{n^{2}}{n+2}}} \times \begin{cases}\mathrm{O}\left(r^{\frac{2 n^{2}}{n+2}-1+\frac{n(n-6)}{n+2}}\right) & \text { if } n=4,5 \text { or }(M, g) \text { l.c.f. } \\
\mathrm{O}\left(r^{8}|\ln r|\right) & \text { if } n=6 \text { and }(M, g) \text { non-l.c.f. } \\
\mathrm{O}\left(r^{\frac{2 n^{2}}{n+2}-1-\frac{n(n-6)}{n+2}}\right) & \text { if } n \geq 7 \text { and }(M, g) \text { non-l.c.f. }\end{cases} \\
= & \begin{array}{ll}
\mathrm{O}\left(\delta_{\varepsilon}(t)^{\frac{2 n(n-2)}{n+2}}\right) & \text { if } n=4,5 \\
\mathrm{O}\left(\delta_{\varepsilon}(t)^{6}\left|\ln \delta_{\varepsilon}(t)\right|\right) & \text { if } n=6 \text { and }(M, g) \text { l.c.f. } \\
\mathrm{O}\left(\delta_{\varepsilon}(t)^{n}\right) & \text { if } n \geq 7 \text { and }(M, g) \text { l.c.f. } \\
\mathrm{O}\left(\delta_{\varepsilon}(t)^{6}\left|\ln \delta_{\varepsilon}(t)\right|^{2}\right) & \text { if } n=6 \text { and }(M, g) \text { non-l.c.f. } \\
\mathrm{O}\left(\delta_{\varepsilon}(t)^{\frac{8 n}{n+2}}\right) & \text { if } n \geq 7 \text { and }(M, g) \text { non-l.c.f. }
\end{array}
\end{aligned}
$$


Moreover, by Lemma A.1, we find that

$$
\int_{B_{\xi}\left(r_{0}\right)}\left|h W_{\varepsilon, t, \xi}\right|^{\frac{2 n}{n+2}} d v_{g}=\mathrm{O}\left(\int_{0}^{r_{0}} \frac{\delta_{\varepsilon}(t)^{\frac{n(n-2)}{n+2}} r^{n-1} d r}{\left(\delta_{\varepsilon}(t)^{2}+r^{2}\right)^{\frac{n(n-2)}{n+2}}}\right)= \begin{cases}\mathrm{O}\left(\delta_{\varepsilon}(t)^{\frac{n(n-2)}{n+2}}\right) & \text { if } n=4,5 \\ \mathrm{O}\left(\delta_{\varepsilon}(t)^{3}\left|\ln \delta_{\varepsilon}(t)\right|\right) & \text { if } n=6 \\ \mathrm{O}\left(\delta_{\varepsilon}(t)^{\frac{4 n}{n+2}}\right) & \text { if } n \geq 7 .\end{cases}
$$

By (3.4), (3.5), (3.11), and (3.12), we get that

$$
\begin{aligned}
& \left\|f_{\varepsilon}\left(W_{\varepsilon, t, \xi}\right)-L_{g} W_{\varepsilon, t, \xi}\right\|_{\frac{2 n}{n+2}} \\
& \quad= \begin{cases}\mathrm{O}\left(\delta_{\varepsilon}(t)^{n-2}+\varepsilon \delta_{\varepsilon}(t)^{\frac{n-2}{2}}\right) & \text { if } n=4,5 \\
\mathrm{O}\left(\delta_{\varepsilon}(t)^{4}\left|\ln \delta_{\varepsilon}(t)\right|^{\frac{2}{3}}+\varepsilon \delta_{\varepsilon}(t)^{2}\left|\ln \delta_{\varepsilon}(t)\right|^{\frac{2}{3}}\right) & \text { if } n=6 \text { and }(M, g) \text { l.c.f. } \\
\mathrm{O}\left(\delta_{\varepsilon}(t)^{\frac{n+2}{2}}+\varepsilon \delta_{\varepsilon}(t)^{2}\right) & \text { if } n \geq 7 \text { and }(M, g) \text { l.c.f. } \\
\mathrm{O}\left(\delta_{\varepsilon}(t)^{4}\left|\ln \delta_{\varepsilon}(t)\right|^{\frac{4}{3}}+\varepsilon \delta_{\varepsilon}(t)^{2}\left|\ln \delta_{\varepsilon}(t)\right|^{\frac{2}{3}}\right) & \text { if } n=6 \text { and }(M, g) \text { non-l.c.f. } \\
\mathrm{O}\left(\delta_{\varepsilon}(t)^{4}+\varepsilon \delta_{\varepsilon}(t)^{2}\right) & \text { if } n \geq 7 \text { and }(M, g) \text { non-l.c.f. }\end{cases}
\end{aligned}
$$

Finally, (2.20) follows from (2.6), (3.3), and (3.13) in view of (3.2). This ends the proof of Proposition 2.2.

\section{THE REDUCED ENERGY}

In Lemma 4.1 below, we give an asymptotic expansion of $J_{\varepsilon}\left(W_{\varepsilon, t, \xi}\right)$ as $\varepsilon \rightarrow 0$, where $J_{\varepsilon}$ is given by (2.21). We let $K_{n}$ be the sharp constant for the embedding of $D^{1,2}\left(\mathbb{R}^{n}\right)$ into $L^{2^{*}}\left(\mathbb{R}^{n}\right)$. It has been proved independently by Rodemich [41, Aubin [2], and Talenti [47] that

$$
K_{n}=\sqrt{\frac{4}{n(n-2) \omega_{n}^{2 / n}}},
$$

where $\omega_{n}$ is the volume of the unit $n$-sphere.

Lemma 4.1. We let $K_{n}$ be as in (4.1), Weyl $g$ be the Weyl curvature tensor of the manifold, and for any $\xi \in M$, we let $A_{\xi}$ be as in (1.5). As $\varepsilon \rightarrow 0$, the following expansions do hold:

(i) when $n=4$,

$$
J_{\varepsilon}\left(W_{\varepsilon, t, \xi}\right)=\frac{1}{4} K_{4}^{-4}+4 \omega_{3} h(\xi) \varepsilon \delta_{\varepsilon}(t)^{2} \ln \frac{1}{\delta_{\varepsilon}(t)}-4 \beta_{4}^{2} A_{\xi} \delta_{\varepsilon}(t)^{2}+\text { h.o.t. }
$$

(ii) when $n=5$ or $(n \geq 6$ and $(M, g)$ is l.c.f.),

$$
J_{\varepsilon}\left(W_{\varepsilon, t, \xi}\right)=\frac{1}{n} K_{n}^{-n}+[n(n-2)]^{\frac{n-2}{2}}\left(\frac{(n-1) \omega_{n}}{2^{n-1}(n-4)} h(\xi) \varepsilon \delta_{\varepsilon}(t)^{2}-\frac{\beta_{n}^{2}}{2} A_{\xi} \delta_{\varepsilon}(t)^{n-2}\right)+\text { h.o.t. }
$$

(iii) when $n=6$ and $(M, g)$ is non-l.c.f.,

$$
J_{\varepsilon}\left(W_{\varepsilon, t, \xi}\right)=\frac{1}{6} K_{6}^{-6}+45 \omega_{6} h(\xi) \varepsilon \delta_{\varepsilon}(t)^{2}-\frac{3}{4} \omega_{6}\left|\mathrm{Weyl}_{g}(\xi)\right|_{g}^{2} \delta_{\varepsilon}(t)^{4} \ln \frac{1}{\delta_{\varepsilon}(t)}+\text { h.o.t. }
$$


(iv) when $n \geq 7$ and $(M, g)$ is non-l.c.f.,

$$
\begin{array}{r}
J_{\varepsilon}\left(W_{\varepsilon, t, \xi}\right)=\frac{1}{n} K_{n}^{-n}+\frac{[n(n-2)]^{\frac{n-2}{2}}}{2^{n-1}(n-4)} \omega_{n}\left((n-1) h(\xi) \varepsilon \delta_{\varepsilon}(t)^{2}-\frac{n-2}{48(n-6)}\left|\mathrm{Weyl}_{g}(\xi)\right|_{g}^{2} \delta_{\varepsilon}(t)^{4}\right) \\
+ \text { h.o.t. }
\end{array}
$$

uniformly with respect to $\xi \in M$ and $t$ in compact subsets of $(0, \infty)$, where h.o.t. stands for a term which is asymptotically smaller than one of the previous terms in the expansion as $\varepsilon \rightarrow 0$.

Proof. All our estimates in this proof are uniform with respect to $\xi \in M, t$ in compact subsets of $(0, \infty)$, and $\varepsilon \in\left(0, \varepsilon_{0}\right)$ for some fixed $\varepsilon_{0}>0$. Since $\widehat{W}_{\varepsilon, t, \xi}$ is a constant and $L_{g_{\xi}} G_{g_{\xi}}(\cdot, \xi)=0$ in $M \backslash B_{\xi}\left(r_{0}\right)$, by (2.2) and (2.5), we get that

$$
\begin{aligned}
\int_{M} \mid & \left.\nabla W_{\varepsilon, t, \xi}\right|_{g} ^{2} d v_{g}+\alpha_{n} \int_{M} \operatorname{Scal}_{g} W_{\varepsilon, t, \xi}^{2} d v_{g} \\
& =\int_{M}\left(L_{g} W_{\varepsilon, t, \xi}\right) W_{\varepsilon, t, \xi} d v_{g}+\int_{\partial B_{\xi}\left(r_{0}\right)}\left(\partial_{\nu_{\mathrm{in}}} W_{\varepsilon, t, \xi}+\partial_{\nu_{\mathrm{out}}} W_{\varepsilon, t, \xi}\right) W_{\varepsilon, t, \xi} d \sigma_{g} \\
& =\int_{M} L_{g_{\xi}}\left(G_{g_{\xi}}(\cdot, \xi) \widehat{W}_{\varepsilon, t, \xi}\right) G_{g_{\xi}}(\cdot, \xi) \widehat{W}_{\varepsilon, t, \xi} d v_{g_{\xi}}+\mathrm{O}\left(\delta_{\varepsilon}(t)^{n}\right) \\
& =\int_{B_{\xi}\left(r_{0}\right)} L_{g_{\xi}}\left(G_{g_{\xi}}(\cdot, \xi) \widehat{W}_{\varepsilon, t, \xi}\right) G_{g_{\xi}}(\cdot, \xi) \widehat{W}_{\varepsilon, t, \xi} d v_{g_{\xi}}+\mathrm{O}\left(\delta_{\varepsilon}(t)^{n}\right)
\end{aligned}
$$

in view of (3.3) and $W_{\varepsilon, t, \xi}=\mathrm{O}\left(\delta_{\varepsilon}(t)^{\frac{n-2}{2}}\right)$ on $\partial B_{\xi}\left(r_{0}\right)$. In the estimates below, we make use of (3.9) along with (2.4), Lemma A.1, and

$$
G_{g_{\xi}}(x, \xi) \widehat{W}_{\varepsilon, t, \xi}(x)=\beta_{n} G_{g_{\xi}}(x, \xi) d_{g_{\xi}}(x, \xi)^{n-2}[n(n-2)]^{\frac{n-2}{4}} \frac{\delta_{\varepsilon}(t)^{\frac{n-2}{2}}}{\left(\delta_{\varepsilon}(t)^{2}+d_{g_{\xi}}(x, \xi)^{2}\right)^{\frac{n-2}{2}}}
$$

for all $x \in B_{\xi}\left(r_{0}\right)$. When $n=4,5$ or $(M, g)$ is l.c.f., we can deduce that

$$
\begin{gathered}
\int_{M}\left|\nabla W_{\varepsilon, t, \xi}\right|_{g}^{2} d v_{g}+\alpha_{n} \int_{M} \operatorname{Scal}_{g} W_{\varepsilon, t, \xi}^{2} d v_{g}=\frac{1}{2}[n(n-2)]^{\frac{n}{2}} \omega_{n-1} I_{n}^{\frac{n-2}{2}} \\
+n^{\frac{n-2}{2}}(n-2)^{\frac{n}{2}} \beta_{n} \omega_{n-1} I_{n}^{n-2} A_{\xi} \delta_{\varepsilon}(t)^{n-2}+\mathrm{O}\left(\delta_{\varepsilon}(t)^{n-1}\right),
\end{gathered}
$$

where we denote $I_{p}^{q}:=\int_{0}^{+\infty}(1+r)^{-p} r^{q} d r$ for all $p, q$ such that $p-q>1$, and we use that $I_{p}^{q}=\frac{q}{p-q-1} I_{p}^{q-1}=\frac{p}{p-q-1} I_{p+1}^{q}$. Concerning the remaining cases, we find that

$$
\begin{aligned}
\int_{M}\left|\nabla W_{\varepsilon, t, \xi}\right|_{g}^{2} d v_{g}+\alpha_{n} \int_{M} \operatorname{Scal}_{g} W_{\varepsilon, t, \xi}^{2} d v_{g}= & 6912 \omega_{5} I_{6}^{2} \\
& -\frac{8}{5} \omega_{5} I_{6}^{4}\left|\operatorname{Weyl}_{g}(\xi)\right|_{g}^{2} \delta_{\varepsilon}(t)^{4} \ln \delta_{\varepsilon}(t)+\mathrm{O}\left(\delta_{\varepsilon}(t)^{4}\right)
\end{aligned}
$$

when $n=6$ and $(M, g)$ is non-l.c.f., and

$$
\begin{aligned}
& \int_{M}\left|\nabla W_{\varepsilon, t, \xi}\right|_{g}^{2} d v_{g}+\alpha_{n} \int_{M} \operatorname{Scal}_{g} W_{\varepsilon, t, \xi}^{2} d v_{g}=\frac{1}{2}[n(n-2)]^{\frac{n}{2}} \omega_{n-1} I_{n}^{\frac{n-2}{2}} \\
& +\frac{n^{\frac{n-2}{2}}(n-2)^{\frac{n+4}{2}}}{48(n+2)(n-1)} \omega_{n-1} I_{n}^{\frac{n+2}{2}} \delta_{\varepsilon}(t)^{4}\left(\frac{\left|\mathrm{Weyl}_{g}(\xi)\right|_{g}^{2}}{12(n-6)}+\frac{1}{n} \Delta_{\mathrm{Eucl}}\left(\operatorname{Scal}_{g_{\xi}} \circ \exp _{\xi}\right)(0)\right)+\mathrm{O}\left(\delta_{\varepsilon}(t)^{5}\right)
\end{aligned}
$$


when $n \geq 7$ and $(M, g)$ is non-l.c.f., in view of symmetry properties. Now, we estimate the term

$$
\begin{aligned}
\int_{M} W_{\varepsilon, t, \xi}^{2^{*}} d v_{g} & =\int_{M}\left(G_{g_{\xi}}(\cdot, \xi) \widehat{W}_{\varepsilon, t, \xi}\right)^{2^{*}} d v_{g_{\xi}} \\
& =[n(n-2)]^{\frac{n}{2}} \int_{B_{0}\left(r_{0}\right)}\left[\beta_{n} G_{g_{\xi}}\left(\exp _{\xi} y, \xi\right)|y|^{n-2}\right]^{2^{*}} \frac{\delta_{\varepsilon}(t)^{n}}{\left(\delta_{\varepsilon}(t)^{2}+|y|^{2}\right)^{n}} d y+\mathrm{O}\left(\delta_{\varepsilon}(t)^{n}\right)
\end{aligned}
$$

in view of (2.4). By Lemma A.1, we get that

$$
\begin{aligned}
& \int_{M} W_{\varepsilon, t, \xi}^{2^{*}} d v_{g}=\frac{1}{2}[n(n-2)]^{\frac{n}{2}} \omega_{n-1} I_{n}^{\frac{n-2}{2}} \\
& + \begin{cases}n^{\frac{n+2}{2}}(n-2)^{\frac{n-2}{2}} \beta_{n} \omega_{n-1} I_{n}^{n-2} A_{\xi} \delta_{\varepsilon}(t)^{n-2}+\mathrm{O}\left(\delta_{\varepsilon}(t)^{n-1}\right) & \text { if } n=4,5 \text { or }(M, g) \text { l.c.f. } \\
-\frac{72}{5} \omega_{5} I_{6}^{4}\left|\mathrm{Weyl}_{g}(\xi)\right|_{g}^{2} \delta_{\varepsilon}(t)^{4} \ln \delta_{\varepsilon}(t)+\mathrm{O}\left(\delta_{\varepsilon}(t)^{4}\right) & \text { if } n=6 \text { and }(M, g) \text { non-l.c.f. } \\
\frac{n^{\frac{n+2}{2}}(n-2)^{\frac{n}{2}}}{48(n-4)(n-1)} \omega_{n-1} I_{n}^{\frac{n+2}{2}} \delta_{\varepsilon}(t)^{4}\left(\frac{\left|\mathrm{Weyl}_{g}(\xi)\right|_{g}^{2}}{12(n-6)}\right. & \text { if } n \geq 7 \text { and }(M, g) \text { non-l.c.f. }\end{cases}
\end{aligned}
$$

by using symmetry properties when $n \geq 7$ and $(M, g)$ is non-l.c.f. Moreover, we find that

$$
\int_{M} h W_{\varepsilon, t, \xi}^{2} d v_{g}= \begin{cases}-8 \omega_{3} h(\xi) \delta_{\varepsilon}(t)^{2} \ln \delta_{\varepsilon}(t)+\mathrm{O}\left(\delta_{\varepsilon}(t)^{2}\right) & \text { if } n=4 \\ \frac{1}{2}[n(n-2)]^{\frac{n-2}{2}} \omega_{n-1} I_{n-2}^{\frac{n-2}{2}} h(\xi) \delta_{\varepsilon}(t)^{2}+\mathrm{O}\left(\delta_{\varepsilon}(t)^{\frac{5}{2}}\right) & \text { if } n \geq 5\end{cases}
$$

as $\varepsilon \rightarrow 0$, in view of $\Lambda_{\xi}(\xi)=1$, (2.4), and Lemma A.1. Successive integrations by parts give that

$$
I_{n}^{\frac{n-2}{2}}=\frac{\omega_{n}}{2^{n-1} \omega_{n-1}}, I_{n}^{n-2}=\frac{1}{n-1}
$$

and (if $n \geq 5$ )

$$
I_{n-2}^{\frac{n-2}{2}}=\frac{(n-1) \omega_{n}}{2^{n-3}(n-4) \omega_{n-1}}, \quad I_{n}^{\frac{n+2}{2}}=\frac{n(n+2) \omega_{n}}{2^{n-1}(n-2)(n-4) \omega_{n-1}} .
$$

Moreover, for any $\xi \in M$, since $g_{\xi}$ defines conformal normal coordinates of order $N \geq 5$, see Lee-Parker [27, Theorem 5.1], and since $\Lambda_{\xi}(\xi)=1$, we get that

$$
\Delta_{\text {Eucl }}\left(\operatorname{Scal}_{g_{\xi}} \circ \exp _{\xi}\right)(0)=\frac{1}{6}\left|\operatorname{Weyl}_{g_{\xi}}(\xi)\right|_{g_{\xi}}^{2}=\frac{1}{6} \mid \text { Weyl }\left._{g}(\xi)\right|_{g} ^{2} .
$$

Finally, (4.2)-(4.5) follow from (4.6)-(4.13) in view of (4.1).

We end this section by proving the validity of the expansion for $\mathcal{J}_{\varepsilon}$ in Proposition 2.3 uniformly with respect to $\xi \in M, t$ in compact subsets of $(0, \infty)$ and $\varepsilon \in\left(0, \varepsilon_{0}\right)$. To this aim, it suffices to prove the (uniform) expansion

$$
\mathcal{J}_{\varepsilon}(t, \xi)=J_{\varepsilon}\left(W_{\varepsilon, t, \xi}\right)+ \begin{cases}\mathrm{o}\left(\delta_{\varepsilon}(t)^{2}\right) & \text { if } n=4 \\ \mathrm{o}\left(\varepsilon \delta_{\varepsilon}(t)^{2}\right) & \text { if } n \geq 5\end{cases}
$$


as $\varepsilon \rightarrow 0$. Indeed, the expansion of $\mathcal{J}_{\varepsilon}$ in Proposition 2.3 follows from combining Lemma 4.1 with (4.14) and letting $\delta_{\varepsilon}(t)$ be as in (2.6). Since

$$
\begin{array}{r}
\mathcal{J}_{\varepsilon}(t, \xi)-J_{\varepsilon}\left(W_{\varepsilon, t, \xi}\right)=\left\langle W_{\varepsilon, t, \xi}-L_{g}^{-1}\left(f_{\varepsilon}\left(W_{\varepsilon, t, \xi}\right)\right), \phi_{\varepsilon, t, \xi}\right\rangle_{L_{g}}+\frac{1}{2}\left(\left\|\phi_{\varepsilon, t, \xi}\right\|_{L_{g}}^{2}+\varepsilon \int_{M} h \phi_{\varepsilon, t, \xi}^{2} d v_{g}\right) \\
-\frac{1}{2^{*}} \int_{M}\left(\left(W_{\varepsilon, t, \xi}+\phi_{\varepsilon, t, \xi}\right)_{+}^{2^{*}}-W_{\varepsilon, t, \xi}^{2^{*}}-2^{*} W_{\varepsilon, t, \xi}^{2^{*}-1} \phi_{\varepsilon, t, \xi}\right) d v_{g}, \quad(4.15)
\end{array}
$$

by Cauchy-Schwarz inequality, we get that

$$
\left|\left\langle W_{\varepsilon, t, \xi}-L_{g}^{-1}\left(f_{\varepsilon}\left(W_{\varepsilon, t, \xi}\right)\right), \phi_{\varepsilon, t, \xi}\right\rangle_{L_{g}}\right| \leq\left\|W_{\varepsilon, t, \xi}-L_{g}^{-1}\left(f_{\varepsilon}\left(W_{\varepsilon, t, \xi}\right)\right)\right\|_{L_{g}}\left\|\phi_{\varepsilon, t, \xi}\right\|_{L_{g}},
$$

and by the Mean Value Theorem and the Hölder's inequality, we get that

$$
\begin{aligned}
& \int_{M}\left(\left(W_{\varepsilon, t, \xi}+\phi_{\varepsilon, t, \xi}\right)_{+}^{2^{*}}-W_{\varepsilon, t, \xi}^{2^{*}}-2^{*} W_{\varepsilon, t, \xi}^{2^{*}-1} \phi_{\varepsilon, t, \xi}\right) d v_{g} \\
& =\mathrm{O}\left(\left\|\phi_{\varepsilon, t, \xi}\right\|_{2^{*}}^{2}\left\|W_{\varepsilon, t, \xi}\right\|_{2^{*}}^{2^{*}-2}+\left\|\phi_{\varepsilon, t, \xi}\right\|_{2^{*}}^{2^{*}}\right)=\mathrm{O}\left(\left\|\phi_{\varepsilon, t, \xi}\right\|_{2^{*}}^{2}+\left\|\phi_{\varepsilon, t, \xi}\right\|_{2^{*}}^{2^{*}}\right) .
\end{aligned}
$$

By Proposition 2.1 and (4.15) - (4.17), it follows that

$$
\mathcal{J}_{\varepsilon}(t, \xi)-J_{\varepsilon}\left(W_{\varepsilon, t, \xi}\right)=\mathrm{O}\left(\left\|R_{\varepsilon, t, \xi}\right\|_{1,2}^{2}\right)
$$

in view of Sobolev's embedding $L^{2^{*}}(M) \hookrightarrow H_{1}^{2}(M)$ and the equivalence between the two norms $\|\cdot\|_{L_{g}}$ and $\|\cdot\|_{1,2}$. Proposition 2.2 now yields that the estimate (4.14) does hold $C^{0}$-uniformly.

\section{Existence of $k$-BUBbles}

The previous analysis can be extended to solutions which have $k$ distinct blow-up points, $k \geq 2$. In the non-l.c.f. case with $n \geq 6$, the "reduced energy" $\widetilde{E}_{k}:(0, \infty)^{k} \times\left(M^{k} \backslash \Delta_{k}\right) \rightarrow \mathbb{R}$, $\Delta_{k}:=\left\{\left(\xi_{1}, \ldots, \xi_{k}\right) \in M^{k}: \xi_{i}=\xi_{j}\right.$ for $\left.i \neq j\right\}$, which governs the location of these blow-up points, is just a super-position of the one for each single point:

$$
\widetilde{E}_{k}(\boldsymbol{t}, \boldsymbol{\xi}):=\sum_{i=1}^{k} \widetilde{E}\left(t_{i}, \xi_{i}\right), \quad \boldsymbol{t}:=\left(t_{1}, \ldots, t_{k}\right) \in(0, \infty)^{k}, \quad \boldsymbol{\xi}:=\left(\xi_{1}, \ldots, \xi_{k}\right) \in M^{k} \backslash \Delta_{k} .
$$

Theorem 2.4 works as well in this context in the following way: as soon as we find $k \operatorname{distinct} C^{p}$ stable, $p=0,1$, critical sets $\widetilde{\mathcal{D}}_{1}, \ldots, \widetilde{\mathcal{D}}_{k}$ of $\widetilde{E}(t, \xi)$, we can construct a family $\left(u_{k, \varepsilon}\right)_{\varepsilon}$ of solutions to (1.3) which blows up, up to a sub-sequence, at points $\left(\xi_{0}\right)_{1}, \ldots,\left(\xi_{0}\right)_{k}$ with $\boldsymbol{\xi}_{0} \in \widetilde{\mathcal{D}}_{1} \times \cdots \times \widetilde{\mathcal{D}}_{k}$ as $\varepsilon \rightarrow 0$. From this result, we deduce Theorem 1.3 exactly as in the case $k=1$.

Theorem 1.2 has its counter-part too: in the non-l.c.f. case with $n \geq 6$, solutions with $k$ blow-up points do exist provided that $k$ is at most the number of isolated critical points of $E(\xi)=h(\xi) \mid$ Weyl $\left._{g}(\xi)\right|_{g} ^{-1}$ with non-trivial local degree and $h>0$.

When $n=4$, the energy for the approximating function (5.2) is not suitable due to the dependence in $t$ of the smallness rate of $\delta_{\varepsilon}(t)$ in (2.6) .

When $n=5$ or $(M, g)$ is l.c.f., the picture is completely different. There is an effective interaction between different blow-up points as expressed by the following "reduced energy" $\widetilde{E}_{k}:(0, \infty)^{k} \times\left(M^{k} \backslash \Delta_{k}\right) \rightarrow \mathbb{R}:$

$$
\widetilde{E}_{k}(\boldsymbol{t}, \boldsymbol{\xi})=c_{2} \sum_{i=1}^{k} t_{i}^{2} h\left(\xi_{i}\right)-c_{3} \sum_{i=1}^{k} t_{i}^{n-2} A_{\xi_{i}}-c_{3} \sum_{\substack{i, j=1 \\ i \neq j}}^{k} t_{i}^{\frac{n-2}{2}} t_{j}^{\frac{n-2}{2}} G_{g}\left(\xi_{i}, \xi_{j}\right),
$$


where $c_{2}, c_{3}>0$ and $A_{\xi}$ is as in (1.5). Theorem 2.4 is still valid in this context by simply replacing $\widetilde{E}(t, \xi)$ with $\widetilde{E}_{k}(\boldsymbol{t}, \boldsymbol{\xi})$. It is no longer possible in general to relate critical sets of $\widetilde{E}_{k}(\boldsymbol{t}, \boldsymbol{\xi})$ with that of an explicit $E_{k}(\boldsymbol{\xi})$ as when $k=1$, with the exception of the case $n=6$ for which we prove Theorem 1.5 with $E_{k}$ defined as in (1.7). In case $n \geq 7$ (in such a way that $\frac{n-2}{2}>2$ ), we can produce a $C^{0}$-stable critical set of $\widetilde{E}_{k}$ through its maximal set, yielding to Theorem 1.4. In this section, we first sketch the proof of Theorem 2.4 in case $k \geq 2$, with $\widetilde{E}(t, \xi)$ replaced by $\widetilde{E}_{k}(\boldsymbol{t}, \boldsymbol{\xi})$, and we then derive from it Theorems 1.4 and 1.5 .

For $\boldsymbol{t}:=\left(t_{1}, \ldots, t_{k}\right) \in(0, \infty)^{k}$ and $\boldsymbol{\xi}:=\left(\xi_{1}, \ldots, \xi_{k}\right) \in M^{k} \backslash \Delta_{k}$, define $\delta_{\varepsilon}\left(t_{i}\right), i=1, \ldots, k$, as in (2.6). The $k$-bubbles approximating function is given by

$$
W_{\varepsilon, \boldsymbol{t}, \boldsymbol{\xi}}:=\sum_{i=1}^{k} W_{\varepsilon, t_{i}, \xi_{i}}
$$

where $W_{\varepsilon, t_{i}, \xi_{i}}$ is defined in (2.7)-(2.8). Since $\Lambda_{\xi}$ are positive functions depending smoothly in $\xi \in M$, there exists $C_{0}>0$ so that $C_{0}^{-1} \leq \Lambda_{\xi}(x) \leq C_{0}$ for all $x, \xi \in M$, and then

$$
C_{0}^{-\frac{2}{n-2}} d_{g}(x, y) \leq d_{g_{\xi}}(x, y) \leq C_{0}^{\frac{2}{n-2}} d_{g}(x, y)
$$

for all $x, y, \xi \in M$. The number $r_{0}$ in (2.8) is also assumed to satisfy $r_{0}<C_{0}^{-\frac{2}{n-2}} \frac{d_{g}\left(\xi_{i}, \xi_{j}\right)}{2}$ for all $i \neq j$ in such a way that $\left\{d_{g_{\xi_{i}}}\left(x, \xi_{i}\right) \leq r_{0}\right\} \cap\left\{d_{g_{\xi_{j}}}\left(x, \xi_{j}\right) \leq r_{0}\right\}=\emptyset$ in view of (5.3). We look for a solution of (1.3) in the form

$$
u_{k, \varepsilon}:=W_{\varepsilon, \boldsymbol{t}, \boldsymbol{\xi}}+\phi_{\varepsilon},
$$

where $\boldsymbol{t} \in(0, \infty)^{k}, \boldsymbol{\xi} \in M^{k} \backslash \Delta_{k}$, and $\phi_{\varepsilon} \in K_{\varepsilon, \boldsymbol{t}, \boldsymbol{\xi}}^{\perp}$ with

$$
K_{\varepsilon, \boldsymbol{t}, \boldsymbol{\xi}}:=\bigcup_{i=1}^{k} K_{\varepsilon, t_{i}, \xi_{i}} \text { and } K_{\varepsilon, \boldsymbol{t}, \boldsymbol{\xi}}^{\perp}:=\bigcap_{i=1}^{k} K_{\varepsilon, t_{i}, \xi_{i}}^{\perp},
$$

where $K_{\varepsilon, t_{i}, \xi_{i}}$ and $K_{\varepsilon, t_{i}, \xi_{i}}^{\perp}$ are as in (2.14)-(2.15). Letting $\Pi_{\varepsilon, \boldsymbol{t}, \boldsymbol{\xi}}$ and $\Pi_{\varepsilon, \boldsymbol{t}, \boldsymbol{\xi}}^{\perp}$ be the respective projections of $H_{1}^{2}(M)$ onto $K_{\varepsilon, \boldsymbol{t}, \boldsymbol{\xi}}$ and $K_{\varepsilon, \boldsymbol{t}, \boldsymbol{\xi}}^{\perp}$, we rewrite equation (1.3) as the system (2.16)(2.17), with $W_{\varepsilon, t, \xi}, \Pi_{\varepsilon, t, \xi}, \Pi_{\varepsilon, t, \xi}^{\perp}$ replaced by $W_{\varepsilon, \boldsymbol{t}, \boldsymbol{\xi}}, \Pi_{\varepsilon, \boldsymbol{t}, \boldsymbol{\xi}}, \Pi_{\varepsilon, \boldsymbol{t}, \boldsymbol{\xi}}^{\perp}$, respectively. We begin with solving equation (2.17) in Proposition 5.1 below, which is, as already observed, a well known result in this context (see for instance Musso-Pistoia [35]).

Proposition 5.1. Given positive real numbers $a<b$ and $\eta$, there exists a positive constant $C=C(a, b, \eta, k, n, M, g, h)$ such that for $\varepsilon>0$ small, for any $\boldsymbol{t} \in[a, b]^{k}$ and $\boldsymbol{\xi} \in M^{k}$ with $d_{g}\left(\xi_{i}, \xi_{j}\right) \geq \eta$ for all $i \neq j$, there exists a unique function $\phi_{\varepsilon, \boldsymbol{t}, \boldsymbol{\xi}} \in K_{\varepsilon, \boldsymbol{t}, \boldsymbol{\xi}}^{\perp}$ which solves equation (2.17) and satisfies

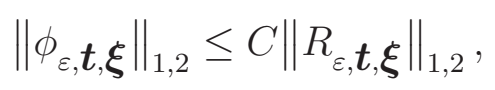

where $R_{\varepsilon, \boldsymbol{t}, \boldsymbol{\xi}}:=W_{\varepsilon, \boldsymbol{t}, \boldsymbol{\xi}}-L_{g}^{-1}\left(f_{\varepsilon}\left(W_{\varepsilon, \boldsymbol{t}, \boldsymbol{\xi}}\right)\right)$. Moreover, $\phi_{\varepsilon, \boldsymbol{t}, \boldsymbol{\xi}}$ is continuously differentiable with respect to $\boldsymbol{t}$ and $\boldsymbol{\xi}$.

We now give an estimate for $\left\|R_{\varepsilon, \boldsymbol{t}, \boldsymbol{\xi}}\right\|_{1,2}$.

Proposition 5.2. Given positive real numbers $a<b$ and $\eta$, there exists a positive constant $C=C(a, b, \eta, k, n, M, g, h)$ such that for $\varepsilon>0$ small, for any $\boldsymbol{t} \in[a, b]^{k}$ and $\boldsymbol{\xi} \in M^{k}$ with 
$d_{g}\left(\xi_{i}, \xi_{j}\right) \geq \eta$ for all $i \neq j$, there holds

$$
\left\|R_{\varepsilon, \boldsymbol{t}, \boldsymbol{\xi}}\right\|_{1,2} \leq C \begin{cases}\varepsilon^{\frac{5}{2}} & \text { if } n=5 \\ \varepsilon^{2}|\ln \varepsilon|^{\frac{2}{3}} & \text { if } n=6 \text { and }(M, g) \text { l.c.f. } \\ \varepsilon^{\frac{n+2}{2(n-4)}} & \text { if } n \geq 7 \text { and }(M, g) \text { l.c.f. }\end{cases}
$$

where $R_{\varepsilon, \boldsymbol{t}, \boldsymbol{\xi}}$ is as in Proposition 5.1 .

Proof. We argue exactly as in the proof of Proposition 2.2. We point out that in this case

$$
\begin{aligned}
\left\langle L_{g}^{-1}\left(f_{\varepsilon}\left(W_{\varepsilon, \boldsymbol{t}, \boldsymbol{\xi}}\right)\right)-W_{\varepsilon, \boldsymbol{t}, \boldsymbol{\xi}}, \phi\right\rangle_{L_{g}}=\sum_{i=1}^{k}\left\langle L_{g}^{-1}\right. & \left.\left(f_{\varepsilon}\left(W_{\varepsilon, t_{i}, \xi_{i}}\right)\right)-W_{\varepsilon, t_{i}, \xi_{i}}, \phi\right\rangle_{L_{g}} \\
& +\int_{M}\left[\left(\sum_{i=1}^{k} W_{\varepsilon, t_{i}, \xi_{i}}\right)^{2^{*}-1}-\sum_{i=1}^{k} W_{\varepsilon, t_{i}, \xi_{i}}^{2^{*}-1}\right] \phi d v_{g},
\end{aligned}
$$

and then

$$
\begin{aligned}
\left\|L_{g}^{-1}\left(f_{\varepsilon}\left(W_{\varepsilon, \boldsymbol{t}, \boldsymbol{\xi}}\right)\right)-W_{\varepsilon, \boldsymbol{t}, \boldsymbol{\xi}}\right\|_{1,2}=\mathrm{O}\left(\sum_{i=1}^{k}\left\|L_{g}^{-1}\left(f_{\varepsilon}\left(W_{\varepsilon, t_{i}, \xi_{i}}\right)\right)-W_{\varepsilon, t_{i}, \xi_{i}}\right\|_{1,2}\right) & \\
& +\mathrm{O}\left(\left\|\left(\sum_{i=1}^{k} W_{\varepsilon, t_{i}, \xi_{i}}\right)^{2^{*}-1}-\sum_{i=1}^{k} W_{\varepsilon, t_{i}, \xi_{i}}^{2^{*}-1}\right\|_{\frac{2 n}{n+2}}\right) .
\end{aligned}
$$

The first $k$ terms are estimated in Proposition 2.2, and the last term can be estimated following the arguments used for (5.9).

For any $\boldsymbol{t} \in(0, \infty)^{k}$ and $\boldsymbol{\xi} \in M^{k} \backslash \Delta_{k}$ we define

$$
\mathcal{J}_{\varepsilon}(\boldsymbol{t}, \boldsymbol{\xi}):=J_{\varepsilon}\left(W_{\varepsilon, \boldsymbol{t}, \boldsymbol{\xi}}+\phi_{\varepsilon, \boldsymbol{t}, \boldsymbol{\xi}}\right),
$$

where $J_{\varepsilon}$ is defined in (2.21) and $\phi_{\varepsilon, \boldsymbol{t}, \boldsymbol{\xi}}$ is given by Proposition 5.1. As already observed for $k=1$, we can solve equation (2.16) by searching critical points of $\mathcal{J}_{\varepsilon}$, and the asymptotic expansion given in Proposition 5.3 below is crucial.

Proposition 5.3. There holds

$$
\mathcal{J}_{\varepsilon}(\boldsymbol{t}, \boldsymbol{\xi})=c_{1}+\varepsilon^{\frac{n-2}{n-4}} \widetilde{E}_{k}(\boldsymbol{t}, \boldsymbol{\xi})+\mathrm{o}\left(\varepsilon^{\frac{n-2}{n-4}}\right)
$$

as $\varepsilon \rightarrow 0$, uniformly with respect to $\boldsymbol{\xi}$ in compact subsets of $M^{k} \backslash \Delta_{k}$ and $\boldsymbol{t}$ in compact subsets of $(0, \infty)^{k}$, where $\widetilde{E}_{k}$ is given by (5.1) and $c_{1}>0$ depends only in $n$.

Proof. We argue exactly as in the proof of Proposition [2.3, taking into account Lemma 5.4 below and exploiting (2.6).

Lemma 5.4. Assume that either $n=5$ or $\left[n \geq 6\right.$ and $(M, g)$ l.c.f.]. We let $K_{n}$ be as in (4.1), $\delta_{\varepsilon}(t)$ be as in (2.6), and $A_{\xi_{i}}$ be as in (1.5). As $\varepsilon \rightarrow 0$, the following expansion does hold

$$
\begin{aligned}
J_{\varepsilon}\left(W_{\varepsilon, \boldsymbol{t}, \boldsymbol{\xi}}\right)=\frac{k}{n} K_{n}^{-n}+[n(n-2)]^{\frac{n-2}{2}} & {\left[\frac{(n-1) \omega_{n}}{2^{n-1}(n-4)} \varepsilon \sum_{i=1}^{k} h\left(\xi_{i}\right) \delta_{\varepsilon}\left(t_{i}\right)^{2}-\frac{\beta_{n}^{2}}{2} \sum_{i=1}^{k} A_{\xi_{i}} \delta_{\varepsilon}\left(t_{i}\right)^{n-2}\right.} \\
& \left.-\frac{\beta_{n}^{2}}{2} \sum_{\substack{i, j=1 \\
i \neq j}}^{k}\left(\delta_{\varepsilon}\left(t_{i}\right) \delta_{\varepsilon}\left(t_{j}\right)\right)^{\frac{n-2}{2}} G_{g}\left(\xi_{i}, \xi_{j}\right)\right]+\mathrm{o}\left(\varepsilon^{\frac{n-2}{n-4}}\right),
\end{aligned}
$$


uniformly with respect to $\boldsymbol{\xi}$ in compact subsets of $M^{k} \backslash \Delta_{k}$ and $\boldsymbol{t}$ in compact subsets of $(0, \infty)^{k}$. Proof. We proceed exactly as in the proof of Lemma 4.1. We only point out that

$$
\begin{aligned}
J_{\varepsilon}\left(W_{\varepsilon, \boldsymbol{t}, \boldsymbol{\xi}}\right) & =\sum_{i=1}^{k} J_{\varepsilon}\left(W_{\varepsilon, t_{i}, \xi_{i}}\right)+\frac{1}{2} \sum_{\substack{i, j=1 \\
i \neq j}}^{k}\left\langle W_{\varepsilon, t_{i}, \xi_{i}}, W_{\varepsilon, t_{j}, \xi_{j}}\right\rangle_{L_{g}} \\
+ & \frac{\varepsilon}{2} \sum_{\substack{i, j=1 \\
i \neq j}}^{k} \int_{M} h W_{\varepsilon, t_{i}, \xi_{i}} W_{\varepsilon, t_{j}, \xi_{j}} d v_{g}-\frac{1}{2^{*}} \int_{M}\left[\left(\sum_{i=1}^{k} W_{\varepsilon, t_{i}, \xi_{i}}\right)^{2^{*}}-\sum_{i=1}^{k} W_{\varepsilon, t_{i}, \xi_{i}}^{2^{*}}\right] d v_{g} .
\end{aligned}
$$

We claim that if $i \neq j$, then

$$
\left\langle W_{\varepsilon, t_{i}, \xi_{i}}, W_{\varepsilon, t_{j}, \xi_{j}}\right\rangle_{L_{g}}=[n(n-2)]^{\frac{n-2}{2}} \beta_{n}^{2}\left(\delta_{\varepsilon}\left(t_{i}\right) \delta_{\varepsilon}\left(t_{j}\right)\right)^{\frac{n-2}{2}} G_{g}\left(\xi_{i}, \xi_{j}\right)+o\left(\varepsilon^{\frac{n-2}{n-4}}\right) .
$$

Indeed, by (2.4), (2.6) $,(3.3),(3.9)$, and Lemma A.1, we get that

$$
\begin{aligned}
\left\langle W_{\varepsilon, t_{i}, \xi_{i}}, W_{\varepsilon, t_{j}, \xi_{j}}\right\rangle_{L_{g}}= & \int_{M}\left(L_{g} W_{\varepsilon, t_{i}, \xi_{i}}\right) W_{\varepsilon, t_{j}, \xi_{j}} d v_{g} \\
& +\int_{\partial B_{\xi_{i}}\left(r_{0}\right)}\left(\partial_{\nu_{\mathrm{in}}} W_{\varepsilon, t_{i}, \xi_{i}}+\partial_{\nu_{\mathrm{out}}} W_{\varepsilon, t_{i}, \xi_{i}}\right) W_{\varepsilon, t_{j}, \xi_{j}} d \sigma_{g} \\
= & \beta_{n} \delta_{\varepsilon}\left(t_{j}\right)^{\frac{2-n}{2}} r_{0}^{n-2} U\left(\delta_{\varepsilon}\left(t_{j}\right)^{-1} r_{0}\right) \\
& \times \int_{B_{\xi_{i}}\left(r_{0}\right)} L_{g_{\xi_{i}}}\left(G_{g_{\xi_{i}}}\left(\cdot, \xi_{i}\right) \widehat{W}_{\varepsilon, t_{i}, \xi_{i}}\right) \Lambda_{\xi_{i}}(x)^{-1} G_{g}\left(x, \xi_{j}\right) d v_{g_{\xi_{i}}}+\mathrm{o}\left(\varepsilon^{\frac{n-2}{n-4}}\right) \\
= & {[n(n-2)]^{\frac{n-2}{4}} \beta_{n} \delta_{\varepsilon}\left(t_{j}\right)^{\frac{n-2}{2}} G_{g}\left(\xi_{i}, \xi_{j}\right) } \\
& \times \int_{B_{0}\left(r_{0}\right)} L_{g_{\xi_{i}}}\left(G_{g_{\xi_{i}}}\left(\cdot, \xi_{i}\right) \widehat{W}_{\varepsilon, t_{i}, \xi_{i}}\right)\left(\exp _{\xi_{i}} y\right)[1+\mathrm{o}(1)+\mathrm{O}(|y|)] d y+\mathrm{o}\left(\varepsilon^{\frac{n-2}{n-4}}\right) \\
= & \frac{1}{2}[n(n-2)]^{\frac{n}{2}} \beta_{n} \omega_{n-1}\left(\delta_{\varepsilon}\left(t_{i}\right) \delta_{\varepsilon}\left(t_{j}\right)\right)^{\frac{n-2}{2}} G_{g}\left(\xi_{i}, \xi_{j}\right) I_{\frac{n+2}{2}}^{\frac{n-2}{2}}+\mathrm{o}\left(\varepsilon^{\frac{n-2}{n-4}}\right)
\end{aligned}
$$

in view of $\Lambda_{\xi_{i}}\left(\xi_{i}\right)=1, L_{g} G_{g}\left(x, \xi_{i}\right)=0$, and

$$
W_{\varepsilon, t_{i}, \xi_{i}}=\beta_{n} G_{g}\left(x, \xi_{i}\right) \delta_{\varepsilon}\left(t_{i}\right)^{\frac{2-n}{2}} r_{0}^{n-2} U\left(\delta_{\varepsilon}\left(t_{i}\right)^{-1} r_{0}\right)
$$

for all $x \in M \backslash B_{\xi_{i}}\left(r_{0}\right)$. Since

$$
I_{\frac{n+2}{2}}^{\frac{n-2}{2}}=\int_{0}^{+\infty} \frac{r^{\frac{n-2}{2}}}{(1+r)^{\frac{n+2}{2}}} d r=\int_{0}^{+\infty}\left(1-\frac{1}{r+1}\right)^{\frac{n-2}{2}} \frac{d r}{(1+r)^{2}}=\int_{0}^{1}(1-s)^{\frac{n-2}{2}} d s=\frac{2}{n}
$$

we deduce the validity of (5.6). We claim that

$$
\begin{aligned}
\frac{1}{2^{*}} \int_{M}\left[\left(\sum_{i=1}^{k} W_{\varepsilon, t_{i}, \xi_{i}}\right)^{2^{*}}\right. & \left.-\sum_{i=1}^{k} W_{\varepsilon, t_{i}, \xi_{i}}^{2^{*}}\right] d v_{g} \\
& =[n(n-2)]^{\frac{n-2}{2}} \beta_{n}^{2} \sum_{\substack{i, j=1 \\
i \neq j}}^{k}\left(\delta_{\varepsilon}\left(t_{i}\right) \delta_{\varepsilon}\left(t_{j}\right)\right)^{\frac{n-2}{2}} G_{g}\left(\xi_{i}, \xi_{j}\right)+\mathrm{o}\left(\varepsilon^{\frac{n-2}{n-4}}\right) .
\end{aligned}
$$


Indeed, by (2.4), (2.6), (5.7), and Lemma A.1, we deduce that

$$
\begin{aligned}
\int_{M} & {\left[\left(\sum_{i=1}^{k} W_{\varepsilon, t_{i}, \xi_{i}}\right)^{2^{*}}-\sum_{i=1}^{k} W_{\varepsilon, t_{i}, \xi_{i}}^{2^{*}}\right] d v_{g} } \\
& =\sum_{j=1}^{k} \int_{B_{\xi_{j}}\left(r_{0}\right)}\left[\left(\sum_{i=1}^{k} W_{\varepsilon, t_{i}, \xi_{i}}\right)^{2^{*}}-W_{\varepsilon, t_{j}, \xi_{j}}^{2^{*}}\right] d v_{g}+\mathrm{o}\left(\varepsilon^{\frac{n-2}{n-4}}\right) \\
& =2^{*}[n(n-2)]^{\frac{n-2}{4}} \beta_{n} \sum_{\substack{i, j=1 \\
i \neq j}}^{k} \delta_{\varepsilon}\left(t_{i}\right)^{\frac{n-2}{2}}(1+\mathrm{o}(1)) \int_{B_{\xi_{j}}\left(r_{0}\right)} G_{g}\left(x, \xi_{i}\right) W_{\varepsilon, t_{j}, \xi_{j}}^{2^{*}-1} d v_{g}+\mathrm{o}\left(\varepsilon^{\frac{n-2}{n-4}}\right) \\
& =\frac{2^{*}}{2}[n(n-2)]^{\frac{n}{2}} \beta_{n} \omega_{n-1} I_{\frac{n+2}{2}}^{\frac{n-2}{2}} \sum_{\substack{i, j=1 \\
i \neq j}}^{k}\left(\delta_{\varepsilon}\left(t_{i}\right) \delta_{\varepsilon}\left(t_{j}\right)\right)^{\frac{n-2}{2}} G_{g}\left(\xi_{j}, \xi_{i}\right)+\mathrm{o}\left(\varepsilon^{\frac{n-2}{n-4}}\right)
\end{aligned}
$$

in view of the estimate $(a+b)^{2^{*}}-a^{2^{*}}-2^{*} a^{2^{*}-1} b=\mathrm{O}\left(a^{2^{*}-2} b^{2}+b^{2^{*}}\right)$ for all $a, b \geq 0$. Therefore, thanks to (5.8), we deduce the validity of (5.9). Moreover, by (2.6) and (5.7), we get that

$$
\begin{aligned}
\int_{M} h W_{\varepsilon, t_{i}, \xi_{i}} W_{\varepsilon, t_{j}, \xi_{j}} d v_{g}=\mathrm{O}\left(\delta_{\varepsilon}\left(t_{j}\right)^{\frac{n-2}{2}} \int_{B_{\xi_{i}}\left(r_{0}\right)}\right. & \left.W_{\varepsilon, t_{i}, \xi_{i}} d v_{g}+\delta_{\varepsilon}\left(t_{i}\right)^{\frac{n-2}{2}} \int_{B_{\xi_{j}}\left(r_{0}\right)} W_{\varepsilon, t_{j}, \xi_{j}} d v_{g}\right) \\
& +\mathrm{O}\left(\delta_{\varepsilon}\left(t_{i}\right)^{\frac{n-2}{2}} \delta_{\varepsilon}\left(t_{j}\right)^{\frac{n-2}{2}}\right)=\mathrm{O}\left(\varepsilon^{\frac{n-2}{n-4}}\right)
\end{aligned}
$$

Inserting (5.6), (5.9), and (5.10) into (5.5) and combining with Lemma 4.1 we finally deduce the validity of (5.4).

Proof of Theorem 1.4. The key point is to show that $\widetilde{E}_{k}$ attains its maximum value

$$
m_{k}:=\sup _{(0, \infty)^{k} \times\left(M^{k} \backslash \Delta_{k}\right)} \widetilde{E}_{k}
$$

at interior points, i.e.

$$
\widetilde{\mathcal{D}}_{k}=\left\{(\boldsymbol{t}, \boldsymbol{\xi}) \in(0, \infty)^{k} \times\left(M^{k} \backslash \Delta_{k}\right): \widetilde{E}_{k}(\boldsymbol{t}, \boldsymbol{\xi})=m_{k}\right\}
$$

is a non-empty compact set. We claim that there exists $L>0$ so that

$$
\sup _{(0, L]^{l} \times\left(M^{l} \backslash \Delta_{l}\right)} \widetilde{E}_{l}=m_{l} \quad \text { and } \sup _{\left((0, \infty)^{l} \backslash(0, L]^{l}\right) \times\left(M^{l} \backslash \Delta_{l}\right)} \widetilde{E}_{l}<m_{l}
$$

for all $l=1, \ldots, k$, and there holds

$$
0<m_{1}<m_{2}<\cdots<m_{k}<+\infty .
$$

Indeed, since $\min \left\{A_{\xi}: \xi \in M\right\}>0$ and

$$
\widetilde{E}_{l}(\boldsymbol{t}, \boldsymbol{\xi}) \leq \sum_{i=1}^{l}\left(c_{2} t_{i}^{2} h\left(\xi_{i}\right)-c_{3} t_{i}^{n-2} A_{\xi_{i}}\right)
$$

for all $(\boldsymbol{t}, \boldsymbol{\xi}) \in(0, \infty)^{k} \times\left(M^{k} \backslash \Delta_{k}\right)$, we have that $\widetilde{E}_{l}(\boldsymbol{t}, \boldsymbol{\xi}) \rightarrow-\infty$ uniformly as soon as $t_{i} \rightarrow+\infty$ for some $i=1, \ldots, l$. Therefore, we can find some $L>0$ large so that (5.11) does hold, and $m_{l}<+\infty$. Since $\max _{M} h>0$, we can find $\xi_{1} \in M$ with $h\left(\xi_{1}\right)>0$, and then 
$c_{2} t_{1}^{2} h\left(\xi_{1}\right)-c_{3} t_{1}^{n-2} A_{\xi_{1}}>0$ for $t_{1}>0$ sufficiently small. It follows that $m_{1}>0$. To conclude the proof of (5.12), observe that for $l \geq 2$, we have that

$$
\begin{aligned}
\widetilde{E}_{l}(\boldsymbol{t}, \boldsymbol{\xi})= & \widetilde{E}_{l-1}\left(t_{1}, \ldots, t_{l-1}, \xi_{1}, \ldots, \xi_{l-1}\right) \\
& +c_{2} t_{l}^{2} h\left(\xi_{l}\right)-c_{3} t_{l}^{n-2} A_{\xi_{l}}-2 c_{3} t_{l}^{\frac{n-2}{2}} \sum_{i=1}^{l-1} t_{i}^{\frac{n-2}{2}} G_{g}\left(\xi_{i}, \xi_{l}\right) .
\end{aligned}
$$

Let $\left(t_{1}^{j}, \ldots, t_{l-1}^{j}, \xi_{1}^{j}, \ldots, \xi_{l-1}^{j}\right)_{j \in \mathbb{N}}$ be a maximizing sequence for $m_{l-1}$. Up to a subsequence, we can assume that $\xi_{i}^{j} \rightarrow \bar{\xi}_{i} \in M$ as $j \rightarrow+\infty$ for all $i=1, \ldots, l-1$. Now, we fix some $\xi_{l} \in\{h>0\} \backslash\left\{\bar{\xi}_{1}, \ldots, \bar{\xi}_{l-1}\right\}$ and we choose $t_{l}>0$ small so that

$$
\begin{aligned}
& c_{2} t_{l}^{2} h\left(\xi_{l}\right)-c_{3} t_{l}^{n-2} A_{\xi_{l}}-2 c_{3} t_{l}^{\frac{n-2}{2}} \sum_{i=1}^{l-1} t_{i}^{\frac{n-2}{2}} G_{g}\left(\bar{\xi}_{i}, \xi_{l}\right) \\
& \quad \geq c_{2} t_{l}^{2} h\left(\xi_{l}\right)-c_{3} t_{l}^{n-2} A_{\xi_{l}}-2 c_{3} L^{\frac{n-2}{2}} t_{l}^{\frac{n-2}{2}} \sum_{i=1}^{l-1} G_{g}\left(\bar{\xi}_{i}, \xi_{l}\right)>0
\end{aligned}
$$

in view of $\frac{n-2}{2}>2$. Therefore, we get that

$$
\begin{aligned}
m_{l} & \geq \lim _{j \rightarrow+\infty} \widetilde{E}_{l}\left(t_{1}^{j}, \ldots, t_{l-1}^{j}, t_{l}^{j}, \xi_{1}^{j}, \ldots, \xi_{l-1}^{j}, \xi_{l}^{j}\right) \\
& =m_{l-1}+c_{2} t_{l}^{2} h\left(\xi_{l}\right)-c_{3} t_{l}^{n-2} A_{\xi_{l}}-2 c_{3} t_{l}^{\frac{n-2}{2}} \sum_{i=1}^{l-1} t_{i}^{\frac{n-2}{2}} G_{g}\left(\bar{\xi}_{i}, \xi_{l}\right)>m_{l-1}
\end{aligned}
$$

and (5.12) is established.

Now, we prove that $\widetilde{\mathcal{D}}_{k} \neq \emptyset$ and that $\widetilde{\mathcal{D}}_{k}$ is a compact set. By (5.11), we can find a maximizing sequence $\left(\boldsymbol{t}^{j}, \boldsymbol{\xi}^{j}\right)_{j \in \mathbb{N}},\left(\boldsymbol{t}^{j}, \boldsymbol{\xi}^{j}\right):=\left(t_{1}^{j}, \ldots, t_{k}^{j}, \xi_{1}^{j}, \ldots, \xi_{k}^{j}\right)$, for $\widetilde{E}_{k}$ so that $t_{i}^{j} \leq L$ for all $i=1, \ldots, k$ and $j \in \mathbb{N}$. By (5.13) with $l=k$, we get that if $t_{k}^{j} \rightarrow 0$ as $j \rightarrow+\infty$, then $m_{k} \leq m_{k-1}$ in contradiction with (5.12). Since the same argument applies for all the $t_{i}^{j}$, s, we get that there exists $\eta>0$ so that $t_{i}^{j} \geq \eta$ for all $i=1, \ldots, k$ and $j \in \mathbb{N}$. By compactness of $[\eta, L]^{k} \times M^{k}$, up to a subsequence, we can assume that $\left(\boldsymbol{t}^{j}, \boldsymbol{\xi}^{j}\right) \rightarrow\left(\boldsymbol{t}_{0}, \boldsymbol{\xi}_{0}\right) \in[\eta, L]^{k} \times M^{k}$ as $j \rightarrow+\infty$. Since $G_{g}(x, y) \rightarrow+\infty$ as $d_{g}(x, y) \rightarrow 0$, we get that $\boldsymbol{\xi}_{0} \in M^{k} \backslash \Delta_{k}$, and thus that $\left(\boldsymbol{t}_{0}, \boldsymbol{\xi}_{0}\right) \in \widetilde{\mathcal{D}}_{k}$. Hence, we get that $\widetilde{\mathcal{D}}_{k} \neq \emptyset$. As for the compactness of $\widetilde{\mathcal{D}}_{k}$, we let $\left(\boldsymbol{t}^{j}, \boldsymbol{\xi}^{j}\right)_{j \in \mathbb{N}}$ be a sequence in $\widetilde{\mathcal{D}}_{k}$, and by the same arguments as above, we deduce that $\left(\boldsymbol{t}^{j}, \boldsymbol{\xi}^{j}\right) \rightarrow\left(\boldsymbol{t}_{0}, \boldsymbol{\xi}_{0}\right) \in[\eta, L]^{k} \times\left(M^{k} \backslash \Delta_{k}\right)$ as $j \rightarrow+\infty$, and by continuity of $\widetilde{E}_{k},\left(\boldsymbol{t}_{0}, \boldsymbol{\xi}_{0}\right) \in \widetilde{\mathcal{D}}_{k}$, which proves that $\widetilde{\mathcal{D}}_{k}$ is a compact set.

To conclude the proof, let $\widetilde{U}$ be a compact neighborhood of $\widetilde{\mathcal{D}}_{k}$ in $(0, \infty)^{k} \times\left(M^{k} \backslash \Delta_{k}\right)$. Since by (5.11) $\widetilde{E}_{k}$ is not a constant function, we have that

$$
\sup _{\partial \widetilde{U}} \widetilde{E}_{k}<\sup _{\widetilde{\mathcal{D}}_{k}} \widetilde{E}_{k}
$$

By Proposition 5.3, we get that

$$
\widetilde{\mathcal{J}}_{\varepsilon}(\boldsymbol{t}, \boldsymbol{\xi}):=\varepsilon^{-\frac{n-2}{n-4}}\left[\mathcal{J}_{\varepsilon}(\boldsymbol{t}, \boldsymbol{\xi})-c_{1}\right] \longrightarrow \widetilde{E}_{k}(\boldsymbol{t}, \boldsymbol{\xi})
$$

as $\varepsilon \rightarrow 0$, uniformly with respect to $\boldsymbol{\xi}$ in compact subsets of $M^{k} \backslash \Delta_{k}$ and $\boldsymbol{t}$ in compact subsets of $(0, \infty)^{k}$. It follows from (5.14) and (5.15) that for $\varepsilon>0$ small, we get that

$$
\sup _{\partial \widetilde{U}} \widetilde{\mathcal{J}}_{\varepsilon}<\sup _{\widetilde{\mathcal{D}}_{k}} \widetilde{\mathcal{J}}_{\varepsilon} .
$$


Then $\widetilde{\mathcal{J}}_{\varepsilon}$ achieves its maximum value in $\widetilde{U}$ at some interior point $\left(\boldsymbol{t}_{\varepsilon}, \boldsymbol{\xi}_{\varepsilon}\right)$ of $\widetilde{U}$, which is a critical point of $\mathcal{J}_{\varepsilon}$ in $\widetilde{U}$. As already observed, we then get that $u_{k, \varepsilon}=W_{\varepsilon, \boldsymbol{t}_{\varepsilon}, \boldsymbol{\xi}_{\varepsilon}}+\phi_{\varepsilon, \boldsymbol{t}_{\varepsilon}, \boldsymbol{\xi}_{\varepsilon}}$ is a critical point of $J_{\varepsilon}$, and thus, by elliptic regularity, a classical solution of (1.3). Up to a subsequence and taking $\widetilde{U}$ smaller and smaller, we can assume that $\left(\boldsymbol{t}_{\varepsilon}, \boldsymbol{\xi}_{\varepsilon}\right) \rightarrow\left(\boldsymbol{t}_{0}, \boldsymbol{\xi}_{0}\right) \in \widetilde{\mathcal{D}}_{k}$ as $\varepsilon \rightarrow 0$.

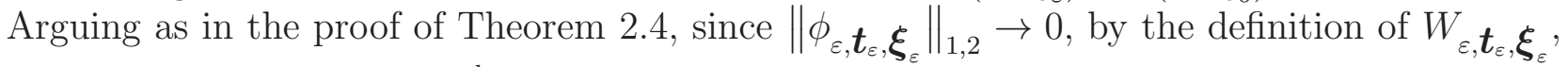
we get that $u_{k, \varepsilon}^{2^{*}} \rightarrow K_{n}^{-n} \sum_{i=1}^{k} \delta_{\left(\xi_{0}\right)_{i}}$ in the measures sense as $\varepsilon \rightarrow 0$. Then the family $\left(u_{k, \varepsilon}\right)_{\varepsilon}$ blows up at the points $\left(\xi_{0}\right)_{1}, \ldots,\left(\xi_{0}\right)_{k}$ as $\varepsilon \rightarrow 0$, where $\left(\boldsymbol{t}_{0}, \boldsymbol{\xi}_{0}\right)$ is so that $\widetilde{E}_{k}\left(\boldsymbol{t}_{0}, \boldsymbol{\xi}_{0}\right)=m_{k}$.

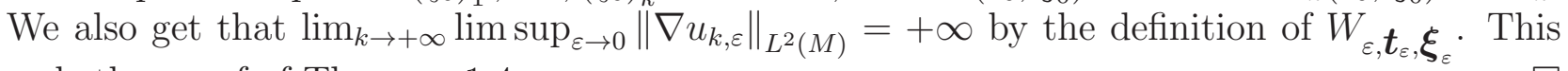
ends the proof of Theorem 1.4 .

Proof of Theorem 1.5. We assume that $n=6$ and $h \in C^{1}(M)$. It is not difficult to show that the expansion (5.4) is $C^{1}$-uniform with respect to $\boldsymbol{t}$ and $\boldsymbol{\xi}$. A straightforward adaptation of the $C^{1}$-estimates in Section 6 below then yields to the $C^{1}$-uniformity of the expansion for $\mathcal{J}_{\varepsilon}$ in Proposition 5.3 for all integers $k \geq 1$. For any $\boldsymbol{\xi}:=\left(\xi_{1}, \ldots, \xi_{k}\right) \in M^{k} \backslash \Delta_{k}$, let $A_{k, \boldsymbol{\xi}}$ and $E_{k}(\boldsymbol{\xi})$ be as in (1.6)-(1.7), and $\boldsymbol{\xi}_{0}:=\left(\left(\xi_{0}\right)_{1}, \ldots,\left(\xi_{0}\right)_{k}\right)$ be an isolated critical point of $E_{k}$ with non-trivial local degree so that $A_{k, \boldsymbol{\xi}_{0}}^{-1} . H$ has positive coordinates. Observe that with these notations, we can write that

$$
\widetilde{E}_{k}(\boldsymbol{t}, \boldsymbol{\xi})=c_{2}\langle\boldsymbol{T}, H(\boldsymbol{\xi})\rangle-c_{3}\left\langle\boldsymbol{T}, A_{k, \boldsymbol{\xi}} \cdot \boldsymbol{T}\right\rangle,
$$

where $\boldsymbol{T}:=\left(t_{1}^{2}, \ldots, t_{k}^{2}\right), H(\boldsymbol{\xi}):=\left(h\left(\xi_{1}\right), \ldots, h\left(\xi_{k}\right)\right)$ and $\langle\cdot, \cdot\rangle$ is the Euclidean scalar product. Arguing as in the proof of Theorem [1.2, it suffices to find $\boldsymbol{t}_{0}\left(\boldsymbol{\xi}_{0}\right) \in(0, \infty)^{k}$ such that $\left(\boldsymbol{t}_{0}\left(\boldsymbol{\xi}_{0}\right), \boldsymbol{\xi}_{0}\right)$ is a $C^{1}$-stable critical point of $\widetilde{E}_{k}$. One then easily checks that such a property is achieved when taking

$$
\boldsymbol{T}_{0}\left(\boldsymbol{\xi}_{0}\right):=\frac{c_{2}}{2 c_{3}} A_{k, \boldsymbol{\xi}_{0}}^{-1} \cdot H\left(\boldsymbol{\xi}_{0}\right) \quad \text { with } \boldsymbol{T}_{0}\left(\boldsymbol{\xi}_{0}\right)=\left(\left(t_{0}\left(\boldsymbol{\xi}_{0}\right)\right)_{1}^{2}, \ldots,\left(t_{0}\left(\boldsymbol{\xi}_{0}\right)\right)_{k}^{2}\right),
$$

which is well defined since $A_{k, \boldsymbol{\xi}_{0}}^{-1} \cdot H\left(\boldsymbol{\xi}_{0}\right)$ has positive coordinates. This ends the proof of Theorem 1.5.

\section{First derivatives estimates}

This section is devoted to the end of the proof of Proposition 2.3. We assume that $h \in$ $C^{1}(M)$ and we prove the $C^{1}$-uniformity of the expansion for $\mathcal{J}_{\varepsilon}$ in Proposition 2.3. Arguing as in the proof of Lemma 4.1, it is not difficult to show that (4.2)-(4.5) are $C^{1}$-uniform with respect to $\xi \in M$ and $t$ in compact subsets of $(0, \infty)$ as $\varepsilon \rightarrow 0$. We only need to prove the $C^{1}$-uniformity of (4.14). We begin with proving the preliminary Lemmas 6.1 6.5. Throughout this section, we identify the tangent spaces $T_{\xi} M$ with $\mathbb{R}^{n}$ thanks to local, smooth, orthonormal frames, so that $\exp _{\xi}$ denotes the composition of the standard exponential map (with respect to $g_{\xi}$ ) with a linear isometry $\Upsilon_{\xi}: \mathbb{R}^{n} \rightarrow T_{\xi} M$ which is smooth with respect to $\xi$. We denote by $\Omega$ the domain in $M$ where the frame is defined. We use the notations

$$
Z_{0, \varepsilon, t, \xi}:=Z_{\varepsilon, t, \xi} \quad \text { and } \quad Z_{i, \varepsilon, t, \xi}:=Z_{\varepsilon, t, \xi, e_{i}}
$$

for all $i=1, \ldots, n$, where $e_{i}$ is the $i$-th vector in the canonical basis of $\mathbb{R}^{n}, Z_{\varepsilon, t, \xi}$ and $Z_{\varepsilon, t, \xi, e_{i}}$ are as in (2.12) -(2.13). We let $W_{\varepsilon, t, \xi}$ be as in (2.7), $J_{\varepsilon}$ be as in (2.21), and $\mathcal{J}_{\varepsilon}$ be as in (2.22). All our estimates in this section are uniform with respect to $t \in[a, b], \xi \in \Omega$, and $\varepsilon \in\left(0, \varepsilon_{0}\right)$ for some fixed $\varepsilon_{0}>0$. In Lemma 6.1 below, we approximate the first derivatives of $W_{\varepsilon, t, \xi}(x)$ with respect to $t, \xi$, and $x$. 
Lemma 6.1. There hold

$$
\begin{aligned}
\left\|\frac{d}{d t} W_{\varepsilon, t, \xi}-\frac{n^{\frac{n-2}{4}}(n-2)^{\frac{n+2}{4}} \delta_{\varepsilon}^{\prime}(t)}{2 \delta_{\varepsilon}(t)} Z_{0, \varepsilon, t, \xi}\right\|_{2^{*}} & =\mathrm{o}(1), \\
\left\|\left.\frac{d}{d \eta_{i}} W_{\varepsilon, t, \exp _{\xi} \eta}\right|_{\eta=0}-\frac{n^{\frac{n-2}{4}}(n-2)^{\frac{n+2}{4}}}{\delta_{\varepsilon}(t)} Z_{i, \varepsilon, t, \xi}\right\|_{2^{*}} & =\mathrm{o}(1)
\end{aligned}
$$

as $\varepsilon \rightarrow 0$ for all $i=1, \ldots, n$, where $\delta_{\varepsilon}(t)$ is as in (2.6).

Proof. We begin with proving (6.2). For any $x \in M$, we find that

$$
\frac{d}{d t} W_{\varepsilon, t, \xi}(x)=G_{g}(x, \xi) \frac{d}{d t} \widehat{W}_{\varepsilon, t, \xi}(x),
$$

where

$$
\begin{aligned}
\frac{d}{d t} \widehat{W}_{\varepsilon, t, \xi}(x) & =\frac{n^{\frac{n-2}{4}}(n-2)^{\frac{n+2}{4}} \delta_{\varepsilon}^{\prime}(t)}{2 \delta_{\varepsilon}(t)^{\frac{n}{2}}} \beta_{n} \times \begin{cases}d_{g_{\xi}}(x, \xi)^{n-2} V_{0}\left(\delta_{\varepsilon}(t)^{-1} \exp _{\xi}^{-1} x\right) & \text { if } d_{g_{\xi}}(x, \xi) \leq r_{0} \\
r_{0}^{n-2} V_{0}\left(\delta_{\varepsilon}(t)^{-1} r_{0}\right) & \text { if } d_{g_{\xi}}(x, \xi)>r_{0}\end{cases} \\
& =\frac{n^{\frac{n-2}{4}}(n-2)^{\frac{n+2}{4}} \delta_{\varepsilon}^{\prime}(t)}{2 \delta_{\varepsilon}(t)} \widehat{Z}_{0, \varepsilon, t, \xi}(x)+\mathrm{O}\left(\delta_{\varepsilon}(t)^{\frac{n-4}{2}} \delta_{\varepsilon}^{\prime}(t) \mathbf{1}_{M \backslash B_{\xi}\left(r_{0} / 2\right)}(x)\right) .
\end{aligned}
$$

(6.2) follows from (2.6), (6.4), and (6.5). Now, we prove (6.3). For any $x \in B_{\xi}\left(r_{0}\right)$, we get that

$$
\left.\frac{d}{d \eta_{i}} W_{\varepsilon, t, \exp _{\xi} \eta}(x)\right|_{\eta=0}=\left.G_{g}(x, \xi) \frac{d}{d \eta_{i}} \widehat{W}_{\varepsilon, t, \exp _{\xi} \eta}(x)\right|_{\eta=0}+\left.\frac{d}{d \eta_{i}} G_{g}\left(x, \exp _{\xi} \eta\right)\right|_{\eta=0} \widehat{W}_{\varepsilon, t, \xi}(x) .
$$

Moreover, letting $y=\exp _{\xi}^{-1} x$ and using Lemma A.2 in appendix, we find that

$$
\left.\frac{d}{d \eta_{i}} \widehat{W}_{\varepsilon, t, \exp _{\xi} \eta}\left(\exp _{\xi} y\right)\right|_{\eta=0}=-n^{\frac{n-2}{4}}(n-2)^{\frac{n+2}{4}} \beta_{n} \frac{\delta_{\varepsilon}(t)^{\frac{n+2}{2}}|y|^{n-4} y_{i}}{\left(\delta_{\varepsilon}(t)^{2}+|y|^{2}\right)^{\frac{n}{2}}}+\mathrm{O}\left(\frac{\delta_{\varepsilon}(t)^{\frac{n+2}{2}}|y|^{n-1}}{\left(\delta_{\varepsilon}(t)^{2}+|y|^{2}\right)^{\frac{n}{2}}}\right)
$$

and, using (2.5), we get that

$$
\left.\frac{d}{d \eta_{i}} G_{g}\left(x, \exp _{\xi} \eta\right)\right|_{\eta=0}=\left.\Lambda_{\xi}(x) \frac{d}{d \eta_{i}} G_{\exp _{\xi} \eta}\left(x, \exp _{\xi} \eta\right)\right|_{\eta=0}+\left.\frac{d}{d \eta_{i}} \Lambda_{\exp _{\xi} \eta}(x)\right|_{\eta=0} G_{g_{\xi}}(x, \xi) .
$$

Since we have chosen $\Lambda_{\xi}$ so that $\Lambda_{\xi}(\xi)=1$ and $\nabla \Lambda_{\xi}(\xi)=0$, we get that

$$
\Lambda_{\xi}\left(\exp _{\xi} y\right)=1+\mathrm{O}\left(|y|^{2}\right) \text { and }\left.\frac{d}{d \eta_{i}} \Lambda_{\exp _{\xi} \eta}\left(\exp _{\xi} y\right)\right|_{\eta=0}=\mathrm{O}(|y|) \text {. }
$$

By (6.8), (6.9), Lemmas A.1 and A.2, we get that

$$
\begin{aligned}
G_{g}\left(\exp _{\xi} y, \xi\right) & =\beta_{n}^{-1}|y|^{2-n}+\mathrm{O}\left(|y|^{4-n}\right), \\
\left.\frac{d}{d \eta_{i}} G_{g}\left(\exp _{\xi} y, \exp _{\xi} \eta\right)\right|_{\eta=0} & =(n-2) \beta_{n}^{-1}|y|^{-n} y_{i}+\mathrm{O}\left(|y|^{3-n}\right) .
\end{aligned}
$$

Moreover, using Lemma A.1, we find that

$$
Z_{i, \varepsilon, t, \xi}\left(\exp _{\xi} y\right)=\frac{\delta_{\varepsilon}(t)^{\frac{n}{2}} \chi(|y|) y_{i}}{\left(\delta_{\varepsilon}(t)^{2}+|y|^{2}\right)^{\frac{n}{2}}}+\mathrm{O}\left(\frac{\delta_{\varepsilon}(t)^{\frac{n}{2}}|y|^{3}}{\left(\delta_{\varepsilon}(t)^{2}+|y|^{2}\right)^{\frac{n}{2}}}\right) \text {. }
$$


By (6.6)-(6.12), we get that

$$
\left.\frac{d}{d \eta_{i}} W_{\varepsilon, t, \exp _{\xi} \eta}\left(\exp _{\xi} y\right)\right|_{\eta=0}=\frac{n^{\frac{n-2}{4}}(n-2)^{\frac{n+2}{4}}}{\delta_{\varepsilon}(t)} Z_{i, \varepsilon, t, \xi}\left(\exp _{\xi} y\right)+\mathrm{O}\left(\frac{\delta_{\varepsilon}(t)^{\frac{n-2}{2}}|y|}{\left(\delta_{\varepsilon}(t)^{2}+|y|^{2}\right)^{\frac{n-2}{2}}}\right)
$$

uniformly with respect to $y \in B_{0}\left(r_{0}\right)$. It follows from (6.13) that

$$
\begin{aligned}
\int_{B_{\xi}\left(r_{0}\right)}\left|\frac{d}{d \eta_{i}} W_{\varepsilon, t, \exp _{\xi} \eta}(x)\right|_{\eta=0}-\left.\frac{n^{\frac{n-2}{4}}(n-2)^{\frac{n+2}{4}}}{\delta_{\varepsilon}(t)} Z_{i, \varepsilon, t, \xi}(x)\right|^{2^{*}} d v_{g} \\
=\mathrm{O}\left(\delta_{\varepsilon}(t)^{n} \int_{0}^{r_{0}} \frac{r^{2^{*}+n-1} d r}{\left(\delta_{\varepsilon}(t)^{2}+r^{2}\right)^{n}}\right)=\mathrm{o}(1)
\end{aligned}
$$

as $\varepsilon \rightarrow 0$. In $M \backslash \overline{B_{\xi}\left(r_{0}\right)}$, we find that

$$
\left.\frac{d}{d \eta_{i}} W_{\varepsilon, t, \exp _{\xi} \eta}(x)\right|_{\eta=0}=\left.\beta_{n} \delta_{\varepsilon}(t)^{\frac{2-n}{2}} r_{0}^{n-2} U\left(\delta_{\varepsilon}(t)^{-1} r_{0}\right) \frac{d}{d \eta_{i}} G_{g}\left(x, \exp _{\xi} \eta\right)\right|_{\eta=0}=\mathrm{O}\left(\delta_{\varepsilon}(t)^{\frac{n-2}{2}}\right)
$$

uniformly with respect to $x \in M \backslash \overline{B_{\xi}\left(r_{0}\right)}$. Finally, (6.3) follows from (6.14) and (6.15). This ends the proof of Lemma 6.1.

In Lemma 6.2 below, we approximate the first derivatives of the energy of our test functions.

Lemma 6.2. There hold

$$
\begin{aligned}
& \frac{d}{d t} J_{\varepsilon}\left(W_{\varepsilon, t, \xi}\right)=\frac{n^{\frac{n-2}{4}}(n-2)^{\frac{n+2}{4}} \delta_{\varepsilon}^{\prime}(t)}{2 \delta_{\varepsilon}(t)} D J_{\varepsilon}\left(W_{\varepsilon, t, \xi}\right) \cdot Z_{0, \varepsilon, t, \xi}+ \begin{cases}\mathrm{o}\left(\delta_{\varepsilon}(t)^{2} \ln \delta_{\varepsilon}(t)\right) & \text { if } n=4 \\
\mathrm{o}\left(\varepsilon \delta_{\varepsilon}(t)^{2}\right) & \text { if } n \geq 5\end{cases} \\
& \left.\frac{d}{d \eta_{i}} J_{\varepsilon}\left(W_{\varepsilon, t, \exp _{\xi} \eta}\right)\right|_{\eta=0}=\frac{n^{\frac{n-2}{4}}(n-2)^{\frac{n+2}{4}}}{\delta_{\varepsilon}(t)} D J_{\varepsilon}\left(W_{\varepsilon, t, \xi}\right) \cdot Z_{i, \varepsilon, t, \xi}+ \begin{cases}\mathrm{o}\left(\delta_{\varepsilon}(t)^{2}\right) & \text { if } n=4 \\
\mathrm{o}\left(\varepsilon \delta_{\varepsilon}(t)^{2}\right) & \text { if } n \geq 5\end{cases}
\end{aligned}
$$

as $\varepsilon \rightarrow 0$ for all $i=1, \ldots, n$, where $\delta_{\varepsilon}(t)$ is as in (2.6).

Proof. We begin with proving (6.16). Integration by parts gives that

$$
\begin{aligned}
\frac{d}{d t} J_{\varepsilon}\left(W_{\varepsilon, t, \xi}\right)-\frac{n^{\frac{n-2}{4}}(n-2)^{\frac{n+2}{4}} \delta_{\varepsilon}^{\prime}(t)}{2 \delta_{\varepsilon}(t)} D & J_{\varepsilon}\left(W_{\varepsilon, t, \xi}\right) \cdot Z_{0, \varepsilon, t, \xi} \\
=\int_{M}\left(L_{g} W_{\varepsilon, t, \xi}-f_{\varepsilon}\left(W_{\varepsilon, t, \xi}\right)\right) & \left(\frac{d}{d t} W_{\varepsilon, t, \xi}-\frac{n^{\frac{n-2}{4}}(n-2)^{\frac{n+2}{4}} \delta_{\varepsilon}^{\prime}(t)}{2 \delta_{\varepsilon}(t)} Z_{0, \varepsilon, t, \xi}\right) d v_{g} \\
& +\int_{\partial B_{\xi}\left(r_{0}\right)}\left(\partial_{\nu_{\text {in }}} W_{\varepsilon, t, \xi}+\partial_{\nu_{\mathrm{out}}} W_{\varepsilon, t, \xi}\right) \frac{d}{d t} W_{\varepsilon, t, \xi} d \sigma_{g} .
\end{aligned}
$$

By (3.4), (3.5), (3.10), and (6.5), we get that

$$
\begin{aligned}
\left(L_{g} W_{\varepsilon, t, \xi}(x)-f_{\varepsilon}\left(W_{\varepsilon, t, \xi}(x)\right)\right) & \left(\frac{d}{d t} W_{\varepsilon, t, \xi}(x)-\frac{n^{\frac{n-2}{4}}(n-2)^{\frac{n+2}{4}} \delta_{\varepsilon}^{\prime}(t)}{2 \delta_{\varepsilon}(t)} Z_{0, \varepsilon, t, \xi}(x)\right) \\
= & \mathrm{O}\left(\left(\delta_{\varepsilon}(t)^{n-1} \delta_{\varepsilon}^{\prime}(t)+\varepsilon \delta_{\varepsilon}(t)^{n-3} \delta_{\varepsilon}^{\prime}(t)\right) \mathbf{1}_{M \backslash B_{\xi}\left(r_{0} / 2\right)}(x)\right) .
\end{aligned}
$$


(6.16) follows from (6.18) and (6.19) in view of (3.3) and (6.5). Now, we prove (6.17). Integration by parts gives that

$$
\begin{aligned}
& \left.\frac{d}{d \eta_{i}} J_{\varepsilon}\left(W_{\varepsilon, t, \exp _{\xi} \eta}\right)\right|_{\eta=0}-\frac{n^{\frac{n-2}{4}}(n-2)^{\frac{n+2}{4}}}{\delta_{\varepsilon}(t)} D J_{\varepsilon}\left(W_{\varepsilon, t, \xi}\right) \cdot Z_{i, \varepsilon, t, \xi} \\
& =\int_{M}\left(L_{g} W_{\varepsilon, t, \xi}-f_{\varepsilon}\left(W_{\varepsilon, t, \xi}\right)\right)\left(\left.\frac{d}{d \eta_{i}} W_{\varepsilon, t, \exp _{\xi} \eta}\right|_{\eta=0}-\frac{n^{\frac{n-2}{4}}(n-2)^{\frac{n+2}{4}}}{\delta_{\varepsilon}(t)} Z_{i, \varepsilon, t, \xi}\right) d v_{g} \\
& +\int_{\partial B_{\xi}\left(r_{0}\right)}\left[\partial_{\nu_{\text {in }}} W_{\varepsilon, t, \xi}\left(\left.\frac{d}{d \eta_{i}} W_{\varepsilon, t, \exp _{\xi} \eta}\right|_{\eta=0}\right)_{\text {in }}+\partial_{\nu_{\text {out }}} W_{\varepsilon, t, \xi}\left(\left.\frac{d}{d \eta_{i}} W_{\varepsilon, t, \exp _{\xi} \eta}\right|_{\eta=0}\right) \text { out }\right] d \sigma_{g}
\end{aligned}
$$

where

$$
\begin{aligned}
\left(\left.\frac{d}{d \eta_{i}} W_{\varepsilon, t, \exp _{\xi} \eta}\right|_{\eta=0}\right)_{\text {in }}(x) & =\left.G_{g}(x, \xi) \frac{d}{d \eta_{i}} \widehat{W}_{\varepsilon, t, \exp _{\xi} \eta}(x)\right|_{\eta=0}+\left.\frac{d}{d \eta_{i}} G_{g}\left(x, \exp _{\xi} \eta\right)\right|_{\eta=0} \widehat{W}_{\varepsilon, t, \xi}(x), \\
\left(\left.\frac{d}{d \eta_{i}} W_{\varepsilon, t, \exp _{\xi} \eta}\right|_{\eta=0}\right)_{\text {out }}(x) & =\left.\frac{d}{d \eta_{i}} G_{g}\left(x, \exp _{\xi} \eta\right)\right|_{\eta=0} \widehat{W}_{\varepsilon, t, \xi}(x)
\end{aligned}
$$

for all $x \in \partial B_{\xi}\left(r_{0}\right)$, in view of (6.6) and (6.15). Regarding the second term in the right hand side of (6.20), on $\partial B_{\xi}\left(r_{0}\right)$, we find that

$$
\begin{aligned}
& \partial_{\nu_{\text {in }}} W_{\varepsilon, t, \xi}\left(\left.\frac{d}{d \eta_{i}} W_{\varepsilon, t, \exp _{\xi} \eta}\right|_{\eta=0}\right)_{\text {in }}+\partial_{\nu_{\text {out }}} W_{\varepsilon, t, \xi}\left(\left.\frac{d}{d \eta_{i}} W_{\varepsilon, t, \exp _{\xi} \eta}\right|_{\eta=0}\right)_{\text {out }} \\
& =\left.\beta_{n} G_{g}(\cdot, \xi) \delta_{\varepsilon}(t)^{\frac{2-n}{2}} \frac{d}{d r}\left(r^{n-2} U\left(\delta_{\varepsilon}(t)^{-1} r\right)\right)\right|_{r=r_{0}}\left(\left.\frac{d}{d \eta_{i}} W_{\varepsilon, t, \exp _{\xi} \eta}\right|_{\eta=0}\right)_{\text {in }} \\
& +\left.\beta_{n} \partial_{\nu_{\text {in }}} G_{g}(\cdot, \xi) \delta_{\varepsilon}(t)^{\frac{2-n}{2}} r_{0}^{n-2} U\left(\delta_{\varepsilon}(t)^{-1} r_{0}\right) G_{g}(x, \xi) \frac{d}{d \eta_{i}} \widehat{W}_{\varepsilon, t, \exp _{\xi} \eta}(x)\right|_{\eta=0}
\end{aligned}
$$

for all $x \in \partial B_{\xi}\left(r_{0}\right)$. By (6.13), we get that

$$
\left(\left.\frac{d}{d \eta_{i}} W_{\varepsilon, t, \exp _{\xi} \eta}\right|_{\eta=0}\right)_{\text {in }}=\mathrm{O}\left(\delta_{\varepsilon}(t)^{\frac{n-2}{2}}\right)
$$

If follows from (6.21)- 6.22 that

$$
\partial_{\nu_{\text {in }}} W_{\varepsilon, t, \xi}\left(\left.\frac{d}{d \eta_{i}} W_{\varepsilon, t, \exp _{\xi} \eta}\right|_{\eta=0}\right)_{\text {in }}+\partial_{\nu_{\text {out }}} W_{\varepsilon, t, \xi}\left(\left.\frac{d}{d \eta_{i}} W_{\varepsilon, t, \exp _{\xi} \eta}\right|_{\eta=0}\right)_{\text {out }}=\mathrm{O}\left(\delta_{\varepsilon}(t)^{n}\right)
$$

in view of (3.3) and (6.7). Now, we estimate the first term in the right hand side of (6.20). In $B_{\xi}\left(r_{0}\right)$, using (3.5), (3.10), and (6.13), we find that

$$
\begin{aligned}
\int_{B_{\xi}\left(r_{0}\right)} & \left(L_{g} W_{\varepsilon, t, \xi}(x)-W_{\varepsilon, t, \xi}(x)^{2^{*}-1}\right)\left(\left.\frac{d}{d \eta_{i}} W_{\varepsilon, t, \exp _{\xi} \eta}(x)\right|_{\eta=0}-\frac{n^{\frac{n-2}{4}}(n-2)^{\frac{n+2}{4}}}{\delta_{\varepsilon}(t)} Z_{i, \varepsilon, t, \xi}(x)\right) d v_{g} \\
= & \delta_{\varepsilon}(t)^{n} \int_{0}^{r_{0}} \frac{r^{n-2} d r}{\left(\delta_{\varepsilon}(t)^{2}+r^{2}\right)^{n-1}} \times \begin{cases}\mathrm{O}\left(r^{n-2}\right) & \text { if } n=4,5 \text { or }(M, g) \text { l.c.f. } \\
\mathrm{O}\left(r^{4} \ln r\right) & \text { if } n=6 \text { and }(M, g) \text { non-l.c.f. } \\
\mathrm{O}\left(r^{4}\right) & \text { if } n \geq 7 \text { and }(M, g) \text { non-l.c.f. }\end{cases} \\
= & \begin{cases}\mathrm{O}\left(\delta_{\varepsilon}(t)^{n-1}\right) & \text { if } n=4,5 \text { or }(M, g) \text { l.c.f. } \\
\mathrm{O}\left(\delta_{\varepsilon}(t)^{5} \ln \delta_{\varepsilon}(t)\right) & \text { if } n=6 \text { and }(M, g) \text { non-l.c.f. } \\
\mathrm{O}\left(\delta_{\varepsilon}(t)^{5}\right) & \text { if } n \geq 7 \text { and }(M, g) \text { non-l.c.f. }\end{cases}
\end{aligned}
$$


and, using (6.13), we find that

$$
\begin{aligned}
& \int_{B_{\xi}\left(r_{0}\right)} h W_{\varepsilon, t, \xi}(x)\left(\left.\frac{d}{d \eta_{i}} W_{\varepsilon, t, \exp _{\xi} \eta}(x)\right|_{\eta=0}-\frac{n^{\frac{n-2}{4}}(n-2)^{\frac{n+2}{4}}}{\delta_{\varepsilon}(t)} Z_{i, \varepsilon, t, \xi}(x)\right) d v_{g} \\
&=\mathrm{O}\left(\delta_{\varepsilon}(t)^{n-2} \int_{0}^{r_{0}} \frac{r^{n} d r}{\left(\delta_{\varepsilon}(t)^{2}+r^{2}\right)^{n-2}}\right)= \begin{cases}\mathrm{O}\left(\delta_{\varepsilon}(t)^{2}\right) & \text { if } n=4 \\
\mathrm{O}\left(\delta_{\varepsilon}(t)^{3}\left|\ln \delta_{\varepsilon}(t)\right|\right) & \text { if } n=5 \\
\mathrm{O}\left(\delta_{\varepsilon}(t)^{3}\right) & \text { if } n \geq 6 .\end{cases}
\end{aligned}
$$

In $M \backslash \overline{B_{\xi}\left(r_{0}\right)}$, using (3.4) and (6.15), we find that

$$
\left(L_{g} W_{\varepsilon, t, \xi}-f_{\varepsilon}\left(W_{\varepsilon, t, \xi}\right)\right)\left(\left.\frac{d}{d \eta_{i}} W_{\varepsilon, t, \exp _{\xi} \eta}\right|_{\eta=0}-\frac{n^{\frac{n-2}{4}}(n-2)^{\frac{n+2}{4}}}{\delta_{\varepsilon}(t)} Z_{i, \varepsilon, t, \xi}\right)=\mathrm{O}\left(\delta_{\varepsilon}(t)^{n}+\varepsilon \delta_{\varepsilon}(t)^{n-2}\right)
$$

uniformly with respect to $x \in M \backslash \overline{B_{\xi}\left(r_{0}\right)}$. Finally, (6.17) follows from (6.20)-(6.26). This ends the proof of Lemma 6.2.

Now, we prove the following error estimates.

Lemma 6.3. There exists a positive constant $C=C(a, b, n, M, g, h)$ such that

$$
\begin{aligned}
& \left\|L_{g}^{-1}\left(f_{\varepsilon}^{\prime}\left(W_{\varepsilon, t, \xi}\right) Z_{0, \varepsilon, t, \xi}\right)-Z_{0, \varepsilon, t, \xi}\right\|_{1,2} \\
& \quad \leq C \begin{cases}\delta_{\varepsilon}(t)^{\frac{n-2}{2}} & \text { if } 4 \leq n \leq 9 \text { or }(M, g) \text { l.c.f. } \\
\delta_{\varepsilon}(t)^{4} & \text { if } n \geq 10 \text { and }(M, g) \text { non-l.c.f. }\end{cases} \\
& \left\|L_{g}^{-1}\left(f_{\varepsilon}^{\prime}\left(W_{\varepsilon, t, \xi}\right) Z_{i, \varepsilon, t, \xi}\right)-Z_{i, \varepsilon, t, \xi}\right\|_{1,2} \\
& \leq C \begin{cases}\delta_{\varepsilon}(t)^{2}\left|\ln \delta_{\varepsilon}(t)\right|^{\frac{3}{4}} & \text { if } n=4 \\
\delta_{\varepsilon}(t)^{\frac{n}{2}} & \text { if } n=5 \text { or }(n \geq 6 \text { and }(M, g) \text { l.c.f. }) \\
\delta_{\varepsilon}(t)^{3}\left|\ln \delta_{\varepsilon}(t)\right|^{\frac{2}{3}} & \text { if } n=6 \text { and }(M, g) \text { non-l.c.f. } \\
\delta_{\varepsilon}(t)^{3} & \text { if } n \geq 7 \text { and }(M, g) \text { non-l.c.f. }\end{cases}
\end{aligned}
$$

for all $i=1, \ldots, n$, where $\delta_{\varepsilon}(t)$ is as in (2.6) .

Proof. For any $i=0, \ldots, n$ and $\phi \in H_{1}^{2}(M)$, an integration by parts gives

$$
\left\langle L_{g}^{-1}\left(f_{\varepsilon}^{\prime}\left(W_{\varepsilon, t, \xi}\right) Z_{i, \varepsilon, t, \xi}\right)-Z_{i, \varepsilon, t, \xi}, \phi\right\rangle_{L_{g}}=\int_{M}\left(f_{\varepsilon}^{\prime}\left(W_{\varepsilon, t, \xi}\right) Z_{i, \varepsilon, t, \xi}-L_{g} Z_{i, \varepsilon, t, \xi}\right) \phi d v_{g} .
$$

By Sobolev's embedding $H_{1}^{2}(M) \hookrightarrow L^{2^{*}}(M)$, it follows from (6.29) that

$$
\left\|L_{g}^{-1}\left(f_{\varepsilon}^{\prime}\left(W_{\varepsilon, t, \xi}\right) Z_{i, \varepsilon, t, \xi}\right)-Z_{i, \varepsilon, t, \xi}\right\|_{1,2}=\mathrm{O}\left(\left\|f_{\varepsilon}^{\prime}\left(W_{\varepsilon, t, \xi}\right) Z_{i, \varepsilon, t, \xi}-L_{g} Z_{i, \varepsilon, t, \xi}\right\|_{\frac{2 n}{n+2}}\right) .
$$

By conformal covariance (2.2) of $L_{g}$ and by (2.5), in $B_{\xi}\left(r_{0}\right)$, we can write that

$$
\begin{aligned}
& f_{\varepsilon}^{\prime}\left(W_{\varepsilon, t, \xi}\right) Z_{i, \varepsilon, t, \xi}-L_{g} Z_{i, \varepsilon, t, \xi} \\
& \quad=\Lambda_{\xi}^{2^{*}-1}\left[\left(2^{*}-1\right) G_{g_{\xi}}(\cdot, \xi)^{2^{*}-1} \widehat{W}_{\varepsilon, t, \xi}^{2^{*}-2} \widehat{Z}_{i, \varepsilon, t, \xi}-L_{g_{\xi}}\left(G_{g_{\xi}}(\cdot, \xi) \widehat{Z}_{i, \varepsilon, t, \xi}\right)\right]-\varepsilon h Z_{i, \varepsilon, t, \xi} .
\end{aligned}
$$

Since $\widehat{Z}_{i, \varepsilon, t, \xi}(\xi)=0$ and $L_{g_{\xi}} G_{g_{\xi}}(\cdot, \xi)=\delta_{\xi}$, we get that

$$
L_{g_{\xi}}\left(G_{g_{\xi}}(\cdot, \xi) \widehat{Z}_{i, \varepsilon, t, \xi}\right)=G_{g_{\xi}}(\cdot, \xi) \Delta_{g_{\xi}} \widehat{Z}_{i, \varepsilon, t, \xi}-2\left\langle\nabla G_{g_{\xi}}(\cdot, \xi), \nabla \widehat{Z}_{i, \varepsilon, t, \xi}\right\rangle_{g_{\xi}} .
$$


We begin with considering the case $i=0$. Since $\widehat{Z}_{0, \varepsilon, t, \xi} \circ \exp _{\xi}$ is radially symmetrical and $V_{0}$ is a solution to the equation $\Delta_{\text {Eucl }} V_{0}=\left(2^{*}-1\right) U^{2^{*}-2} V_{0}$ in $\mathbb{R}^{n}$, writing $\Delta_{g_{\xi}} \widehat{Z}_{0, \varepsilon, t, \xi}\left(\exp _{\xi} y\right)$ in polar coordinates and using (2.4), we find that

$$
\begin{aligned}
G_{g_{\xi}} & \left(\exp _{\xi} y, \xi\right) \Delta_{g_{\xi}} \widehat{Z}_{0, \varepsilon, t, \xi}\left(\exp _{\xi} y\right) \\
= & G_{g_{\xi}}\left(\exp _{\xi} y, \xi\right) \Delta_{\operatorname{Eucl}}\left(\widehat{Z}_{0, \varepsilon, t, \xi} \circ \exp _{\xi}\right)(y)+\mathrm{O}\left(|y|^{N-n+1}\left|\nabla\left(\widehat{Z}_{0, \varepsilon, t, \xi} \circ \exp _{\xi}\right)(y)\right|\right) \\
= & \left(2^{*}-1\right) \beta_{n} \delta_{\varepsilon}(t)^{-\frac{n+2}{2}} \chi|y|^{n-2} G_{g_{\xi}}\left(\exp _{\xi} y, \xi\right) U\left(\frac{|y|}{\delta_{\varepsilon}(t)}\right)^{2^{*}-2} V_{0}\left(\frac{y}{\delta_{\varepsilon}(t)}\right) \\
& -2(n-2) \beta_{n} \delta_{\varepsilon}(t)^{\frac{n+2}{2}} \chi|y|^{n-4} G_{g_{\xi}}\left(\exp _{\xi} y, \xi\right) \frac{(n+2)|y|^{2}-(n-2) \delta_{\varepsilon}(t)^{2}}{\left(\delta_{\varepsilon}(t)^{2}+|y|^{2}\right)^{\frac{n+2}{2}}} \\
& +\mathrm{O}\left(\frac{\delta_{\varepsilon}(t)^{\frac{n+2}{2}}|y|^{N-2}}{\left(\delta_{\varepsilon}(t)^{2}+|y|^{2}\right)^{\frac{n}{2}}}+\delta_{\varepsilon}(t)^{\frac{n-2}{2}}\right)
\end{aligned}
$$

uniformly with respect to $y \in B_{0}\left(r_{0}\right)$. Moreover, since $\widehat{Z}_{0, \varepsilon, t, \xi} \circ \exp _{\xi}$ is radially symmetrical, we get that

$$
\begin{aligned}
& \left\langle\nabla G_{g_{\xi}}\left(\exp _{\xi} y, \xi\right), \nabla \widehat{Z}_{0, \varepsilon, t, \xi}\left(\exp _{\xi} y\right)\right\rangle_{g_{\xi}}=\partial_{r}\left[G_{g_{\xi}}\left(\exp _{\xi} y, \xi\right)\right] \partial_{r}\left[\widehat{Z}_{0, \varepsilon, t, \xi} \circ \exp _{\xi}\right] \\
& \quad=\beta_{n} \delta_{\varepsilon}(t)^{\frac{n+2}{2}} \chi|y|^{n-3} \partial_{r}\left[G_{g_{\xi}}\left(\exp _{\xi} y, \xi\right)\right] \frac{(n+2)|y|^{2}-(n-2) \delta_{\varepsilon}(t)^{2}}{\left(\delta_{\varepsilon}(t)^{2}+|y|^{2}\right)^{\frac{n+2}{2}}}+\mathrm{O}\left(\delta_{\varepsilon}(t)^{\frac{n-2}{2}}\right) .
\end{aligned}
$$

By (6.32)- (6.34) and Lemma A.1, we get that

$$
\begin{aligned}
& \left(2^{*}-1\right) G_{g_{\xi}}\left(\exp _{\xi} y, \xi\right)^{2^{*}-1} \widehat{W}_{\varepsilon, t, \xi}\left(\exp _{\xi} y\right)^{2^{*}-2} \widehat{Z}_{0, \varepsilon, t, \xi}\left(\exp _{\xi} y\right)-L_{g_{\xi}}\left(G_{g_{\xi}}(\cdot, \xi) \widehat{Z}_{0, \varepsilon, t, \xi}\right)\left(\exp _{\xi} y\right) \\
& \quad=\mathrm{O}\left(\delta_{\varepsilon}(t)^{\frac{n-2}{2}}\right)+\frac{\delta_{\varepsilon}(t)^{\frac{n+2}{2}}}{\left(\delta_{\varepsilon}(t)^{2}+|y|^{2}\right)^{\frac{n}{2}}} \times \begin{cases}\mathrm{O}\left(|y|^{n-4}\right) & \text { if } n=4,5 \text { or }(M, g) \text { l.c.f. } \\
\mathrm{O}\left(|y|^{2} \ln |y|\right) & \text { if } n=6 \text { and }(M, g) \text { non-l.c.f. } \\
\mathrm{O}\left(|y|^{2}\right) & \text { if } n \geq 7 \text { and }(M, g) \text { non-l.c.f., }\end{cases}
\end{aligned}
$$

which, inserted into (6.30)- (6.31), yields to the validity of (6.27) in view of (2.6). Now, we consider the case $i=1, \ldots, n$. Using (2.4), we find that

$$
\begin{aligned}
\Delta_{g_{\xi}} \widehat{Z}_{i, \varepsilon, t, \xi}\left(\exp _{\xi} y\right)= & \Delta_{\operatorname{Eucl}}\left(\widehat{Z}_{i, \varepsilon, t, \xi} \circ \exp _{\xi}\right)(y)-\beta_{n} \chi(|y|) \partial_{j} g_{\xi}^{i j}\left(\exp _{\xi} y\right) \frac{\delta_{\varepsilon}(t)^{\frac{n}{2}}|y|^{n-2}}{\left(\delta_{\varepsilon}(t)^{2}+|y|^{2}\right)^{\frac{n}{2}}} \\
& +\mathrm{O}\left(\frac{\delta_{\varepsilon}(t)^{\frac{n}{2}}|y|^{N+n-3}}{\left(\delta_{\varepsilon}(t)^{2}+|y|^{2}\right)^{\frac{n}{2}}}+|y|^{N}\left|\nabla\left(\chi(|y|) \frac{\delta_{\varepsilon}(t)^{\frac{n}{2}}|y|^{n-2}}{\left(\delta_{\varepsilon}(t)^{2}+|y|^{2}\right)^{\frac{n}{2}}}\right)\right|\right)
\end{aligned}
$$

uniformly with respect to $y \in B_{0}\left(r_{0}\right)$, where $\partial_{j} g_{\xi}^{i j}$ are the derivatives of the components of $g_{\xi}^{-1}$ in geodesic normal coordinates. Since $g_{\xi}$ defines conformal normal coordinates of order $N \geq 3$, see Lee-Parker [27, Theorem 5.1 and Lemma 5.5], we get that the Ricci curvature $\operatorname{Ric}_{g_{\xi}}$ of $g_{\xi}$ vanishes at $\xi$, and thus

$$
\partial_{j} g_{\xi}^{i j}\left(\exp _{\xi} y\right)= \begin{cases}0 & \text { if }(M, g) \text { l.c.f. } \\ -\frac{1}{3}\left(\operatorname{Ric}_{g_{\xi}}\right)_{i p}(\xi) y^{p}+\mathrm{O}\left(|y|^{2}\right)=\mathrm{O}\left(|y|^{2}\right) & \text { if }(M, g) \text { non-l.c.f. }\end{cases}
$$


Since $V_{i}$ is a solution of $\Delta_{\text {Eucl }} V_{i}=\left(2^{*}-1\right) U^{2^{*}-2} V_{i}$ in $\mathbb{R}^{n}$, by (6.35)

$$
\begin{aligned}
G_{g_{\xi}} & \left(\exp _{\xi} y, \xi\right) \Delta_{g_{\xi}} \widehat{Z}_{i, \varepsilon, t, \xi}\left(\exp _{\xi} y\right) \\
= & \left(2^{*}-1\right) \beta_{n} \delta_{\varepsilon}(t)^{-\frac{n+2}{2}} \chi|y|^{n-2} G_{g_{\xi}}\left(\exp _{\xi} y, \xi\right) U\left(\frac{|y|}{\delta_{\varepsilon}(t)}\right)^{2^{*}-2} V_{i}\left(\frac{y}{\delta_{\varepsilon}(t)}\right) \\
& +2(n-2) \beta_{n} \delta_{\varepsilon}(t)^{\frac{n}{2}} \chi|y|^{n-4} y_{i} G_{g_{\xi}}\left(\exp _{\xi} y, \xi\right) \frac{|y|^{2}-(n-1) \delta_{\varepsilon}(t)^{2}}{\left(\delta_{\varepsilon}(t)^{2}+|y|^{2}\right)^{\frac{n+2}{2}}} \\
& +\mathrm{O}\left(\delta_{\varepsilon}(t)^{\frac{n}{2}}+\frac{\delta_{\varepsilon}(t)^{\frac{n}{2}}|y|^{\alpha}}{\left(\delta_{\varepsilon}(t)^{2}+|y|^{2}\right)^{\frac{n}{2}}}\right)
\end{aligned}
$$

where $\alpha=N-2$ if $(M, g)$ is l.c.f. and $\alpha=2$ is $(M, g)$ is not l.c.f. Moreover, we get that

$$
\begin{aligned}
& \left\langle\nabla G_{g_{\xi}}\left(\exp _{\xi} y, \xi\right), \nabla \widehat{Z}_{i, \varepsilon, t, \xi}\left(\exp _{\xi} y\right)\right\rangle_{g_{\xi}} \\
& =y_{i} \partial_{r}\left[G_{g_{\xi}}\left(\exp _{\xi} y, \xi\right)\right] \partial_{r}\left[\frac{\beta_{n} \chi \delta_{\varepsilon}(t)^{\frac{n}{2}}|y|^{n-2}}{\left(\delta_{\varepsilon}(t)^{2}+|y|^{2}\right)^{\frac{n}{2}}}\right]+\partial_{y_{i}}\left[G_{g_{\xi}}\left(\exp _{\xi} y, \xi\right)\right] \frac{\beta_{n} \chi \delta_{\varepsilon}(t)^{\frac{n}{2}}|y|^{n-2}}{\left(\delta_{\varepsilon}(t)^{2}+|y|^{2}\right)^{\frac{n}{2}}} \\
& =-\beta_{n} \delta_{\varepsilon}(t)^{\frac{n}{2}} \chi(|y|) \partial_{r}\left[G_{g_{\xi}}\left(\exp _{\xi} y, \xi\right)\right]|y|^{n-3} y_{i} \frac{2|y|^{2}-(n-2) \delta_{\varepsilon}(t)^{2}}{\left(\delta_{\varepsilon}(t)^{2}+|y|^{2}\right)^{\frac{n+2}{2}}} \\
& +\beta_{n} \delta_{\varepsilon}(t)^{\frac{n}{2}} \chi(|y|) \partial_{y_{i}}\left[G_{g_{\xi}}\left(\exp _{\xi} y, \xi\right)\right] \frac{\delta_{\varepsilon}(t)^{\frac{n}{2}}|y|^{n-2}}{\left(\delta_{\varepsilon}(t)^{2}+|y|^{2}\right)^{\frac{n}{2}}}+\mathrm{O}\left(\delta_{\varepsilon}(t)^{\frac{n}{2}}\right) .
\end{aligned}
$$

By (6.32), 6.37)-(6.38) and Lemma A.1, we get that

$$
\begin{array}{r}
\left(2^{*}-1\right) G_{g_{\xi}}\left(\exp _{\xi} y, \xi\right)^{2^{*}-1} \widehat{W}_{\varepsilon, t, \xi}\left(\exp _{\xi} y\right)^{2^{*}-2} \widehat{Z}_{i, \varepsilon, t, \xi}\left(\exp _{\xi} y\right)-L_{g_{\xi}}\left(G_{g_{\xi}}(\cdot, \xi) \widehat{Z}_{i, \varepsilon, t, \xi}\right)\left(\exp _{\xi} y\right) \\
=\mathrm{O}\left(\delta_{\varepsilon}(t)^{\frac{n}{2}}\right)+\frac{\delta_{\varepsilon}(t)^{\frac{n}{2}}}{\left(\delta_{\varepsilon}(t)^{2}+|y|^{2}\right)^{\frac{n}{2}}} \times \begin{cases}\mathrm{O}\left(|y|^{n-3}\right) & \text { if } n=4,5 \text { or }(M, g) \text { l.c.f. } \\
\mathrm{O}\left(|y|^{2}\right) & \text { if } n \geq 6 \text { and }(M, g) \text { non-l.c.f. }\end{cases}
\end{array}
$$

which, inserted into (6.30)-(6.31), yields to the validity of (6.28) in view of (2.6). This ends the proof of Lemma 6.3.

By Proposition [2.1, for $\varepsilon$ small, for any $t \in[a, b]$ and $\xi \in M$, there exist $\lambda_{\varepsilon, t, \xi} \in \mathbb{R}$ and $\omega_{\varepsilon, t, \xi} \in T_{\xi} M$ such that

$$
D J_{\varepsilon}\left(W_{\varepsilon, t, \xi}+\phi_{\varepsilon, t, \xi}\right)=\left\langle\lambda_{\varepsilon, t, \xi} Z_{\varepsilon, t, \xi}+Z_{\varepsilon, t, \xi, \omega_{\varepsilon, t, \xi}} \cdot\right\rangle_{L_{g}},
$$

where $Z_{\varepsilon, t, \xi}$ and $Z_{\varepsilon, t, \xi, \omega_{\varepsilon, t, \xi}}$ are as in (2.11). We let $Z_{0, \varepsilon, t, \xi}, \ldots, Z_{n, \varepsilon, t, \xi} \in \mathbb{R}$ be as in (6.1). We let $\lambda_{0, \varepsilon, t, \xi}, \ldots, \lambda_{n, \varepsilon, t, \xi} \in \mathbb{R}$ be such that

$$
\lambda_{0, \varepsilon, t, \xi}:=\lambda_{\varepsilon, t, \xi} \quad \text { and } \quad \sum_{i=1}^{n} \lambda_{i, \varepsilon, t, \xi} e_{i}:=\omega_{\varepsilon, t, \xi}
$$

where $\lambda_{\varepsilon, t, \xi}$ and $\omega_{\varepsilon, t, \xi}$ are as in (6.39). We estimate the $\lambda_{i, \varepsilon, t, \xi}$ 's in Lemma 6.4 below.

Lemma 6.4. For any $i=0, \ldots, n$, in case $n \geq 5$, there holds

$$
\lambda_{i, \varepsilon, t, \xi}=\frac{D J_{\varepsilon}\left(W_{\varepsilon, t, \xi}\right) \cdot Z_{i, \varepsilon, t, \xi}}{\left\|\nabla V_{i}\right\|_{2}^{2}}+ \begin{cases}\mathrm{o}\left(\varepsilon \delta_{\varepsilon}(t)^{2}\right) & \text { if } i=0 \\ \mathrm{o}\left(\varepsilon \delta_{\varepsilon}(t)^{3}\right) & \text { if } i=1, \ldots, n\end{cases}
$$


and in case $n=4$, there holds

$$
\lambda_{i, \varepsilon, t, \xi}=\frac{D J_{\varepsilon}\left(W_{\varepsilon, t, \xi}\right) \cdot Z_{0, \varepsilon, t, \xi}}{\left\|\nabla V_{0}\right\|_{2}^{2}}+ \begin{cases}\mathrm{o}\left(\delta_{\varepsilon}(t)^{2}\right) & \text { if } i=0 \\ \mathrm{O}\left(\varepsilon^{2} \delta_{\varepsilon}(t)^{2}\right) & \text { if } i=1, \ldots, n\end{cases}
$$

as $\varepsilon \rightarrow 0$, where $\delta_{\varepsilon}(t)$ is as in (2.6). In particular, there holds

$$
\lambda_{i, \varepsilon, t, \xi}= \begin{cases}\mathrm{O}\left(\delta_{\varepsilon}(t)^{2} \ln \left|\delta_{\varepsilon}(t)\right|\right) & \text { if } i=0 \text { and } n=4 \\ \mathrm{O}\left(\delta_{\varepsilon}(t)^{2}\right) & \text { if } i=1, \ldots, n \text { and } n=4 \\ \mathrm{O}\left(\varepsilon \delta_{\varepsilon}(t)^{2}\right) & \text { if } n \geq 5 .\end{cases}
$$

Proof. For any $i=0, \ldots, n$, by (6.39) - (6.40), we get that

$$
D J_{\varepsilon}\left(W_{\varepsilon, t, \xi}+\phi_{\varepsilon, t, \xi}\right) \cdot Z_{i, \varepsilon, t, \xi}=\sum_{j=0}^{n} \lambda_{j, \varepsilon, t, \xi}\left\langle Z_{i, \varepsilon, t, \xi}, Z_{j, \varepsilon, t, \xi}\right\rangle_{L_{g}} .
$$

For any $i, j=0, \ldots, n$, we find that

$$
\left\langle Z_{i, \varepsilon, t, \xi}, Z_{j, \varepsilon, t, \xi}\right\rangle_{L_{g}}=\left\|\nabla V_{i}\right\|_{2}^{2} \delta_{i j}+\mathrm{o}\left(\delta_{\varepsilon}(t)\right)
$$

as $\varepsilon \rightarrow 0$, where the $\delta_{i j}$ 's are the Kronecker symbols. It follows from (6.44)-(6.45) that

$$
D J_{\varepsilon}\left(W_{\varepsilon, t, \xi}+\phi_{\varepsilon, t, \xi}\right) \cdot Z_{i, \varepsilon, t, \xi}=\lambda_{i, \varepsilon, t, \xi}\left\|\nabla V_{i}\right\|_{2}^{2}+\mathrm{o}\left(\delta_{\varepsilon}(t) \sum_{j=0}^{n}\left|\lambda_{j, \varepsilon, t, \xi}\right|\right)
$$

as $\varepsilon \rightarrow 0$. Independently, we get that

$$
\begin{array}{r}
D J_{\varepsilon}\left(W_{\varepsilon, t, \xi}+\phi_{\varepsilon, t, \xi}\right) \cdot Z_{i, \varepsilon, t, \xi}=D J_{\varepsilon}\left(W_{\varepsilon, t, \xi}\right) \cdot Z_{i, \varepsilon, t, \xi}+\left\langle Z_{i, \varepsilon, t, \xi}-L_{g}^{-1}\left(f_{\varepsilon}^{\prime}\left(W_{\varepsilon, t, \xi}\right) Z_{i, \varepsilon, t, \xi}\right), \phi_{\varepsilon, t, \xi}\right\rangle_{L_{g}} \\
-\int_{M}\left(f_{0}\left(W_{\varepsilon, t, \xi}+\phi_{\varepsilon, t, \xi}\right)-f_{0}\left(W_{\varepsilon, t, \xi}\right)-f_{0}^{\prime}\left(W_{\varepsilon, t, \xi}\right) \phi_{\varepsilon, t, \xi}\right) Z_{i, \varepsilon, t, \xi} d v_{g} \cdot \quad(6.47)
\end{array}
$$

By Cauchy-Schwarz inequality, we get that

$$
\left|\left\langle Z_{i, \varepsilon, t, \xi}-L_{g}^{-1}\left(f_{\varepsilon}^{\prime}\left(W_{\varepsilon, t, \xi}\right) Z_{i, \varepsilon, t, \xi}\right), \phi_{\varepsilon, t, \xi}\right\rangle_{L_{g}}\right| \leq\left\|Z_{i, \varepsilon, t, \xi}-L_{g}^{-1}\left(f_{\varepsilon}^{\prime}\left(W_{\varepsilon, t, \xi}\right) Z_{i, \varepsilon, t, \xi}\right)\right\|_{L_{g}}\left\|\phi_{\varepsilon, t, \xi}\right\|_{L_{g}} .
$$

By the Mean Value Theorem and Hölder's inequality, we get that

$$
\begin{aligned}
\int_{M}( & \left.f_{0}\left(W_{\varepsilon, t, \xi}+\phi_{\varepsilon, t, \xi}\right)-f_{0}\left(W_{\varepsilon, t, \xi}\right)-f_{0}^{\prime}\left(W_{\varepsilon, t, \xi}\right) \phi_{\varepsilon, t, \xi}\right) Z_{i, \varepsilon, t, \xi} d v_{g} \\
& = \begin{cases}\mathrm{O}\left(\left(\left\|W_{\varepsilon, t, \xi}\right\|_{2^{*}}^{2^{*}-3}+\left\|\phi_{\varepsilon, t, \xi}\right\|_{2^{*}}^{2^{*}-3}\right)\left\|\phi_{\varepsilon, t, \xi}\right\|_{2^{*}}^{2}\left\|Z_{i, \varepsilon, t, \xi}\right\|_{2^{*}}\right) & \text { if } n=4,5 \\
\mathrm{O}\left(\left\|W_{\varepsilon, t, \xi}^{2^{*}-3} Z_{i, \varepsilon, t, \xi}\right\|_{2^{*}}\left\|\phi_{\varepsilon, t, \xi}\right\|_{2^{*}}^{2}\right) & \text { if } n \geq 6 .\end{cases} \\
& =\mathrm{O}\left(\left\|\phi_{\varepsilon, t, \xi}\right\|_{2^{*}}^{2}\right) .
\end{aligned}
$$

By (2.6), 6.47)-(6.49), Propositions 2.1, 2.2, and Lemma 6.3, we get that in case $n \geq 5$, there holds

$$
D J_{\varepsilon}\left(W_{\varepsilon, t, \xi}+\phi_{\varepsilon, t, \xi}\right) \cdot Z_{0, \varepsilon, t, \xi}=D J_{\varepsilon}\left(W_{\varepsilon, t, \xi}\right) \cdot Z_{i, \varepsilon, t, \xi}+ \begin{cases}\mathrm{o}\left(\varepsilon \delta_{\varepsilon}(t)^{2}\right) & \text { if } i=0 \\ \mathrm{o}\left(\varepsilon \delta_{\varepsilon}(t)^{3}\right) & \text { if } i=1, \ldots, n\end{cases}
$$

and in case $n=4$, there holds

$$
D J_{\varepsilon}\left(W_{\varepsilon, t, \xi}+\phi_{\varepsilon, t, \xi}\right) \cdot Z_{0, \varepsilon, t, \xi}=D J_{\varepsilon}\left(W_{\varepsilon, t, \xi}\right) \cdot Z_{i, \varepsilon, t, \xi}+ \begin{cases}\mathrm{o}\left(\delta_{\varepsilon}(t)^{2}\right) & \text { if } i=0 \\ \mathrm{O}\left(\varepsilon^{2} \delta_{\varepsilon}(t)^{2}\right) & \text { if } i=1, \ldots, n\end{cases}
$$


as $\varepsilon \rightarrow 0$. Finally, (6.41) and (6.42) follow from (6.46), (6.50), and (6.51). The estimate (6.43) follows from (6.41) - (6.42) and the validity of (4.2) -(4.5) in a $C^{1}$-uniform way with respect to $\xi \in M$ and $t$ in compact subsets of $(0, \infty)$ as $\varepsilon \rightarrow 0$. This ends the proof of Lemma 6.4.

We then prove the following result.

Lemma 6.5. For any $i=1, \ldots, n$, there hold

$$
\begin{aligned}
\left.\frac{d}{d \eta_{i}} \mathcal{J}_{\varepsilon}\left(t, \exp _{\xi} \eta\right)\right|_{\eta=0} & =\frac{n^{\frac{n-2}{4}}(n-2)^{\frac{n+2}{4}}}{\delta_{\varepsilon}(t)}\left\|\nabla V_{i}\right\|_{2}^{2} \lambda_{i, \varepsilon, t, \xi}+\mathrm{o}\left(\sum_{j=0}^{n}\left|\lambda_{j, \varepsilon, t, \xi}\right|\right) \\
\frac{d}{d t} \mathcal{J}_{\varepsilon}(t, \xi) & =\frac{n^{\frac{n-2}{4}}(n-2)^{\frac{n+2}{4}} \delta_{\varepsilon}^{\prime}(t)}{2 \delta_{\varepsilon}(t)}\left\|\nabla V_{0}\right\|_{2}^{2} \lambda_{0, \varepsilon, t, \xi}+\mathrm{o}\left(\sum_{j=0}^{n}\left|\lambda_{j, \varepsilon, t, \xi}\right|\right)
\end{aligned}
$$

as $\varepsilon \rightarrow 0$, where $\delta_{\varepsilon}(t)$ is as in (2.6).

Proof. For any $i=1, \ldots, n$, by (6.39)

$$
\left.\frac{d}{d \eta_{i}} \mathcal{J}_{\varepsilon}\left(t, \exp _{\xi} \eta\right)\right|_{\eta=0}=\sum_{j=0}^{n} \lambda_{j, \varepsilon, t, \xi}\left\langle Z_{j, \varepsilon, t, \xi},\left.\frac{d}{d \eta_{i}}\left(W_{\varepsilon, t, \exp _{\xi} \eta}+\phi_{\varepsilon, t, \exp _{\xi} \eta}\right)\right|_{\eta=0}\right\rangle_{L_{g}} .
$$

For any $i=1, \ldots, n$ and $j=0, \ldots, n$, an integration by parts gives that

$$
\begin{aligned}
& \left\langle Z_{j, \varepsilon, t, \xi},\left.\frac{d}{d \eta_{i}} W_{\varepsilon, t, \exp _{\xi} \eta}\right|_{\eta=0}\right\rangle_{L_{g}}=\int_{M} L_{g} Z_{j, \varepsilon, t, \xi}\left(\left.\frac{d}{d \eta_{i}} W_{\varepsilon, t, \exp _{\xi} \eta}\right|_{\eta=0}-\frac{n^{\frac{n-2}{4}}(n-2)^{\frac{n+2}{4}}}{\delta_{\varepsilon}(t)} Z_{i, \varepsilon, t, \xi}\right) d v_{g} \\
& +\frac{n^{\frac{n-2}{4}}(n-2)^{\frac{n+2}{4}}}{\delta_{\varepsilon}(t)}\left\langle Z_{j, \varepsilon, t, \xi}, Z_{i, \varepsilon, t, \xi}\right\rangle_{L_{g}} \text {. }
\end{aligned}
$$

By Hölder's inequality and (6.3), we get that

$$
\begin{gathered}
\int_{M} L_{g} Z_{j, \varepsilon, t, \xi}\left(\left.\frac{d}{d \eta_{i}} W_{\varepsilon, t, \exp _{\xi} \eta}\right|_{\eta=0}-\frac{n^{\frac{n-2}{4}}(n-2)^{\frac{n+2}{4}}}{\delta_{\varepsilon}(t)} Z_{i, \varepsilon, t, \xi}\right) d v_{g} \leq\left\|L_{g} Z_{j, \varepsilon, t, \xi}\right\|_{\frac{2 n}{n+2}} \\
\times\left\|\left.\frac{d}{d \eta_{i}} W_{\varepsilon, t, \exp _{\xi} \eta}\right|_{\eta=0}-\frac{n^{\frac{n-2}{4}}(n-2)^{\frac{n+2}{4}}}{\delta_{\varepsilon}(t)} Z_{i, \varepsilon, t, \xi}\right\|_{2^{*}}=\mathrm{o}\left(\left\|L_{g} Z_{j, \varepsilon, t, \xi}\right\|_{\frac{2 n}{n+2}}\right)=\mathrm{o}(1)
\end{gathered}
$$

as $\varepsilon \rightarrow 0$. It follows from $(\underline{6.45})$ and $(\underline{6.55})-(\underline{6.56})$ that

$$
\left\langle Z_{j, \varepsilon, t, \xi},\left.\frac{d}{d \eta_{i}} W_{\varepsilon, t, \exp _{\xi} \eta}\right|_{\eta=0}\right\rangle_{L_{g}}=\frac{n^{\frac{n-2}{4}}(n-2)^{\frac{n+2}{4}}}{\delta_{\varepsilon}(t)}\left\|\nabla V_{i}\right\|_{2}^{2} \delta_{i j}+\mathrm{o}(1)
$$

as $\varepsilon \rightarrow 0$, where the $\delta_{i j}$ 's are the Kronecker symbols. Since $\phi_{\varepsilon, t, \xi}$ belongs to $K_{\varepsilon, t, \xi}^{\perp}$, differentiating the equation $\left\langle Z_{j, \varepsilon, t, \xi}, \phi_{\varepsilon, t, \xi}\right\rangle_{L_{g}}=0$, we find that

$$
\left\langle Z_{j, \varepsilon, t, \xi},\left.\frac{d}{d \eta_{i}} \phi_{\varepsilon, t, \exp _{\xi} \eta}\right|_{\eta=0}\right\rangle_{L_{g}}=-\left\langle\left.\frac{d}{d \eta_{i}} Z_{j, \varepsilon, t, e x p_{\xi} \eta}\right|_{\eta=0}, \phi_{\varepsilon, t, \xi}\right\rangle_{L_{g}} .
$$

By (6.58), Cauchy-Schwarz inequality, Propositions 2.1 and 2.2, we get that

$$
\begin{aligned}
\left|\left\langle Z_{j, \varepsilon, t, \xi},\left.\frac{d}{d \eta_{i}} \phi_{\varepsilon, t, \exp _{\xi} \eta}\right|_{\eta=0}\right\rangle_{L_{g}}\right| & \leq\left\|\left.\frac{d}{d \eta_{i}} Z_{j, \varepsilon, t, \exp _{\xi} \eta}\right|_{\eta=0}\right\|_{L_{g}}\left\|\phi_{\varepsilon, t, \xi}\right\|_{L_{g}} \\
& =\mathrm{O}\left(\delta_{\varepsilon}(t)^{-1}\left\|\phi_{\varepsilon, t, \xi}\right\|_{1,2}\right)=\mathrm{o}(1)
\end{aligned}
$$

as $\varepsilon \rightarrow 0$. (6.52) follows from (6.54), (6.57), and (6.59). (6.53) follows from similar arguments by using (6.2). This ends the proof of Lemma 6.5. 
Proof of the $C^{1}$-uniformity of (4.14) with respect to $t$. The result follows directly from Lemmas 6.2, 6.4, and 6.5.

Proof of the $C^{1}$-uniformity of (4.14) with respect to $\xi$. In case $n \geq 5$, the result follows directly from Lemmas 6.2, 6.4, and 6.5. The case $n=4$ is trickier. In this case, the estimate (6.42) is not sufficient to get such a direct proof as in higher dimensions. We prove the result in case $n=4$ in what follows. For any $i=1, \ldots, n$ and $x \in M$, we define

$$
Y_{i, \varepsilon, t, \xi}(x):=\chi\left(d_{g_{\xi}}(x, \xi)\right) \Lambda_{\xi}(x) \partial_{y_{i}}\left[\widetilde{W}_{\varepsilon, t, \xi} \circ \exp _{\xi}+\widetilde{\phi}_{\varepsilon, t, \xi} \circ \exp _{\xi}\right]\left(\exp _{\xi}^{-1} x\right),
$$

where $\widetilde{W}_{\varepsilon, t, \xi}:=W_{\varepsilon, t, \xi} / \Lambda_{\xi}$ and $\widetilde{\phi}_{\varepsilon, t, \xi}:=\phi_{\varepsilon, t, \xi} / \Lambda_{\xi}$. We claim that

$$
D J_{\varepsilon}\left(W_{\varepsilon, t, \xi}+\phi_{\varepsilon, t, \xi}\right) \cdot Y_{i, \varepsilon, t, \xi}=-\frac{n^{\frac{n-2}{4}}(n-2)^{\frac{n+2}{4}}}{\delta_{\varepsilon}(t)}\left\|\nabla V_{i}\right\|_{2}^{2} \lambda_{i, \varepsilon, t, \xi}+\mathrm{o}\left(\sum_{j=0}^{n}\left|\lambda_{j, \varepsilon, t, \xi}\right|\right) .
$$

It follows from (6.39) -6.40$)$ and (6.45) that in order to prove (6.60), it suffices to prove that for any $i=1, \ldots, n$ and $j=0, \ldots, n$, there holds

$$
\left\langle Z_{j, \varepsilon, t, \xi}, Y_{i, \varepsilon, t, \xi}+\frac{n^{\frac{n-2}{4}}(n-2)^{\frac{n+2}{4}}}{\delta_{\varepsilon}(t)} Z_{i, \varepsilon, t, \xi}\right\rangle_{L_{g}}=\mathrm{o}(1)
$$

as $\varepsilon \rightarrow 0$. Integrating by parts and applying Hölder's inequality, we get that

$$
\begin{aligned}
&\left\langle Z_{j, \varepsilon, t, \xi}, Y_{i, \varepsilon, t, \xi}+\frac{n^{\frac{n-2}{4}}(n-2)^{\frac{n+2}{4}}}{\delta_{\varepsilon}(t)} Z_{i, \varepsilon, t, \xi}\right\rangle_{L_{g}}=\int_{M} L_{g} Z_{j, \varepsilon, t, \xi}\left(Y_{i, \varepsilon, t, \xi}+\frac{n^{\frac{n-2}{4}}(n-2)^{\frac{n+2}{4}}}{\delta_{\varepsilon}(t)} Z_{i, \varepsilon, t, \xi}\right) d v_{g} \\
& \leq\left\|L_{g} Z_{j, \varepsilon, t, \xi}\right\|_{\frac{2 n}{n+2}}\left\|\chi\left(d_{g_{\xi}}(\cdot, \xi)\right) \Lambda_{\xi} \partial_{y_{i}}\left[\widetilde{W}_{\varepsilon, t, \xi} \circ \exp _{\xi}\right] \circ \exp _{\xi}^{-1}+\frac{n^{\frac{n-2}{4}}(n-2)^{\frac{n+2}{4}}}{\delta_{\varepsilon}(t)} Z_{i, \varepsilon, t, \xi}\right\|_{2^{*}} \\
&+\left\|L_{g} Z_{j, \varepsilon, t, \xi}\right\|_{2}\left\|\chi\left(d_{g_{\xi}}(\cdot, \xi)\right) \Lambda_{\xi} \nabla \widetilde{\phi}_{\varepsilon, t, \xi}\right\|_{2} \cdot \quad(6.62)
\end{aligned}
$$

Using similar arguments as in the proof of Lemma 6.1, we find that

$$
\left\|\chi\left(d_{g_{\xi}}(\cdot, \xi)\right) \Lambda_{\xi} \partial_{y_{i}}\left[\widetilde{W}_{\varepsilon, t, \xi} \circ \exp _{\xi}\right] \circ \exp _{\xi}^{-1}+\frac{n^{\frac{n-2}{4}}(n-2)^{\frac{n+2}{4}}}{\delta_{\varepsilon}(t)} Z_{i, \varepsilon, t, \xi}\right\|_{2^{*}}=\mathrm{o}(1)
$$

as $\varepsilon \rightarrow 0$. Rough estimates give that

$$
\left\|L_{g} Z_{j, \varepsilon, t, \xi}\right\|_{\frac{2 n}{n+2}}=\mathrm{O}(1) \quad \text { and } \quad\left\|L_{g} Z_{j, \varepsilon, t, \xi}\right\|_{2}=\mathrm{O}\left(\delta_{\varepsilon}(t)^{-1}\right) .
$$

Moreover, by (2.6), and Propositions 2.1 and 2.2, we get that in dimension $n=4$, there holds

$$
\left\|\chi\left(d_{g_{\xi}}(\cdot, \xi)\right) \Lambda_{\xi} \nabla \widetilde{\phi}_{\varepsilon, t, \xi}\right\|_{2}=\mathrm{O}\left(\left\|\widetilde{\phi}_{\varepsilon, t, \xi}\right\|_{1,2}\right)=\mathrm{O}\left(\left\|\phi_{\varepsilon, t, \xi}\right\|_{1,2}\right)=\mathrm{O}\left(\left\|\phi_{\varepsilon, t, \xi}\right\|_{L_{g}}\right)=\mathrm{O}\left(\varepsilon \delta_{\varepsilon}(t)\right) \text {. }
$$

(6.61) follows from (6.62)-(6.65), and thus we get (6.60). It follows from (6.2), (6.60), and Lemmas 6.4 and 6.5 that in dimension $n=4$, there holds

$$
\left.\frac{d}{d \eta_{i}} \mathcal{J}_{\varepsilon}\left(t, \exp _{\xi} \eta\right)\right|_{\eta=0}=-D J_{\varepsilon}\left(W_{\varepsilon, t, \xi}+\phi_{\varepsilon, t, \xi}\right) \cdot Y_{i, \varepsilon, t, \xi}+\mathrm{o}\left(\delta_{\varepsilon}(t)^{2} \ln \left|\delta_{\varepsilon}(t)\right|\right)
$$

as $\varepsilon \rightarrow 0$. By the conformal change of metric $g_{\xi}=\Lambda_{\xi}^{2^{*}-2} g$, we get that

$$
\begin{aligned}
D J_{\varepsilon}( & \left.W_{\varepsilon, t, \xi}+\phi_{\varepsilon, t, \xi}\right) \cdot Y_{i, \varepsilon, t, \xi}=\int_{M}\left(\left\langle\nabla\left(\widetilde{W}_{\varepsilon, t, \xi}+\widetilde{\phi}_{\varepsilon, t, \xi}\right), \nabla \widetilde{Y}_{i, \varepsilon, t, \xi}\right\rangle_{g_{\xi}}\right. \\
& \left.+\left(\alpha_{n} \operatorname{Scal}_{g_{\xi}}+\varepsilon \Lambda_{\xi}^{2-2^{*}} h\right)\left(\widetilde{W}_{\varepsilon, t, \xi}+\widetilde{\phi}_{\varepsilon, t, \xi}\right) \widetilde{Y}_{i, \varepsilon, t, \xi}-\left(\widetilde{W}_{\varepsilon, t, \xi}+\widetilde{\phi}_{\varepsilon, t, \xi}\right)_{+}^{2^{*}-1} \widetilde{Y}_{i, \varepsilon, t, \xi}\right) d v_{g_{\xi}}
\end{aligned}
$$


where $\widetilde{Y}_{i, \varepsilon, t, \xi}:=Y_{i, \varepsilon, t, \xi} / \Lambda_{\xi}$. Since $\widetilde{Y}_{i, \varepsilon, t, \xi} \equiv 0$ in $M \backslash B_{\xi}\left(r_{0}\right)$, letting $y=\exp _{\xi}^{-1} x$ and integrating by parts (6.67), we find that

$$
D J_{\varepsilon}\left(W_{\varepsilon, t, \xi}+\phi_{\varepsilon, t, \xi}\right) \cdot Y_{i, \varepsilon, t, \xi}=I_{1, i, \varepsilon, t, \xi}+I_{2, i, \varepsilon, t, \xi}+I_{3, i, \varepsilon, t, \xi}+I_{4, i, \varepsilon, t, \xi},
$$

where

$$
\begin{aligned}
I_{1, i, \varepsilon, t, \xi}:= & D J_{\varepsilon}\left(W_{\varepsilon, t, \xi}\right) \cdot\left(\chi\left(d_{g_{\xi}}(\cdot, \xi)\right) \Lambda_{\xi} \partial_{y_{i}}\left[\widetilde{W}_{\varepsilon, t, \xi} \circ \exp _{\xi}\right] \circ \exp _{\xi}^{-1}\right) \\
I_{2, i, \varepsilon, t, \xi}:= & \int_{B_{0}\left(r_{0}\right)}\left\{g_{\xi}^{p q} \partial_{y_{p}} \chi \partial_{y_{q}}\left[\widetilde{\phi}_{\varepsilon, t, \xi} \circ \exp _{\xi}\right] \partial_{y_{i}}\left[\widetilde{W}_{\varepsilon, t, \xi} \circ \exp _{\xi}\right]\left|\exp _{\xi}^{*} g_{\xi}\right|\right. \\
& +g_{\xi}^{p q} \partial_{y_{p}} \chi \partial_{y_{q}}\left[\widetilde{W}_{\varepsilon, t, \xi} \circ \exp _{\xi}\right] \partial_{y_{i}}\left[\widetilde{\phi}_{\varepsilon, t, \xi} \circ \exp _{\xi}\right]\left|\exp _{\xi}^{*} g_{\xi}\right| \\
& -\chi \partial_{y_{i}}\left[g_{\xi}^{p q}\right] \partial_{y_{p}}\left[\widetilde{W}_{\varepsilon, t, \xi} \circ \exp _{\xi}\right] \partial_{y_{q}}\left[\widetilde{\phi}_{\varepsilon, t, \xi} \circ \exp _{\xi}\right]\left|\exp _{\xi}^{*} g_{\xi}\right| \\
& -\chi\left(\widetilde{W}_{\varepsilon, t, \xi} \circ \exp _{\xi}\right)\left(\widetilde{\phi}_{\varepsilon, t, \xi} \circ \exp _{\xi}\right) \partial_{y_{i}}\left[\left(\alpha_{n} \operatorname{Scal}_{g_{\xi}}+\varepsilon \Lambda_{\xi}^{2-2^{*}} h\right) \circ \exp _{\xi}\right]\left|\exp _{\xi}^{*} g_{\xi}\right| \\
& -\left(\left\langle\nabla \widetilde{W}_{\varepsilon, t, \xi}, \nabla \widetilde{\phi}_{\varepsilon, t, \xi}\right\rangle_{g_{\xi}}+\left(\alpha_{n} \operatorname{Scal}_{g_{\xi}}+\varepsilon \Lambda_{\xi}^{2-2^{*}} h\right) \widetilde{W}_{\varepsilon, t, \xi} \widetilde{\phi}_{\varepsilon, t, \xi}-\widetilde{W}_{\varepsilon, t, \xi}^{2^{*}-1} \widetilde{\phi}_{\varepsilon, t, \xi}\right) \circ \exp _{\xi} \\
I_{3, i, \varepsilon, t, \xi}:= & \int_{B_{0}\left(r_{0}\right)}\left\{g_{\xi}^{p q} \partial_{y_{p}} \chi \partial_{y_{q}}\left[\widetilde{\phi}_{\varepsilon, t, \xi} \circ \exp _{\xi}\right] \partial_{y_{i}}\left[\widetilde{\phi}_{\varepsilon, t, \xi} \circ \exp _{\xi}\right]\left|\exp _{\xi}^{*} g_{\xi}\right|\right. \\
& \left.\left.-\frac{1}{2} \chi \partial_{y_{i}}\left[g_{\xi}^{p q}\right] \partial_{y_{p}}\left[\widetilde{\phi}_{\varepsilon, t, \xi} \circ \exp _{\xi}\right] \partial_{y_{q}}\left[\widetilde{\phi}_{\varepsilon, t, \xi} \circ \exp _{\xi}\right]\left|\exp _{\xi}^{*} g_{\xi}\right|\right]\right\} d y, \\
& -\frac{1}{2} \chi\left(\widetilde{\phi}_{\varepsilon, t, \xi} \circ \exp _{\xi}\right)^{2} \partial_{y_{i}}\left[\left(\alpha_{n} \operatorname{Scal}_{g_{\xi}}+\varepsilon \Lambda_{\xi}^{2-2^{*}} h\right) \circ \exp _{\xi}\right]\left|\exp _{\xi}^{*} g_{\xi}\right| \\
& \left.-\frac{1}{2}\left(\left|\nabla \widetilde{\phi}_{\varepsilon, t, \xi}\right|_{g_{\xi}}^{2}+\left(\alpha_{n} \operatorname{Scal}_{g_{\xi}}+\varepsilon \Lambda_{\xi}^{2-2^{*}} h\right) \widetilde{\phi}_{\varepsilon, t, \xi}^{2}\right) \circ \exp _{\xi} \partial_{y_{i}}\left[\chi\left|\exp _{\xi}^{*} g_{\xi}\right|\right]\right\} d y, \\
I_{4, i, \varepsilon, t, \xi}:= & \frac{1}{2^{*}} \int_{B_{0}\left(r_{0}\right)}\left\{\left(\left(\widetilde{W}_{\varepsilon, t, \xi}+\widetilde{\phi}_{\varepsilon, t, \xi}\right)_{+}^{2^{*}}-\widetilde{W}_{\varepsilon, t, \xi}^{2^{*}}-2^{*} \widetilde{W}_{\varepsilon, t, \xi}^{2^{*}-1} \widetilde{\phi}_{\varepsilon, t, \xi}\right) \circ \exp _{\xi}\right. \\
& \left.\times \partial_{y_{i}}\left[\chi\left|\exp _{\xi}^{*} g_{\xi}\right|\right]\right\} d y,
\end{aligned}
$$

where $g_{\xi}^{p q}$ are the components of $g_{\xi}^{-1}$ in geodesic normal coordinates. Using similar arguments as in the proof of Lemma 6.2. we find that

$$
I_{1, i, \varepsilon, t, \xi}=-\left.\frac{d}{d \eta_{i}} J_{\varepsilon}\left(W_{\varepsilon, t, \xi}\right)\right|_{\eta=0}+\mathrm{o}\left(\delta_{\varepsilon}(t)^{2}\right)
$$

as $\varepsilon \rightarrow 0$. Now, we estimate $I_{2, i, \varepsilon, t, \xi}$. Since $g_{\xi}$ defines conformal normal coordinates of order $N \geq 5$, see Lee-Parker [27, Theorem 5.1], we get that

$$
\partial_{y_{i}}\left[\operatorname{Scal}_{g_{\xi}} \circ \exp _{\xi}\right](y)=\mathrm{O}(|y|), \quad \partial_{y_{i}}\left[\left|\exp _{\xi}^{*} g_{\xi}\right|\right](y)=\mathrm{O}\left(|y|^{N-1}\right) .
$$

Since $\partial_{y_{i}}\left[g_{\xi}^{p q}\right](y)=\mathrm{O}(|y|)$, by using Hölder's inequality and (6.70), we find that

$$
\begin{array}{r}
I_{2, i, \varepsilon, t, \xi}=\mathrm{O}\left(\left\|d_{g_{\xi}}(\cdot, \xi) \nabla \widetilde{W}_{\varepsilon, t, \xi}\right\|_{2}\left\|\nabla \widetilde{\phi}_{\varepsilon, t, \xi}\right\|_{2}+\left(\left\|d_{g_{\xi}}(\cdot, \xi) \widetilde{W}_{\varepsilon, t, \xi}\right\|_{\frac{2 n}{n+2}}\right.\right. \\
\left.\left.\quad+\varepsilon\left\|\widetilde{W}_{\varepsilon, t, \xi}\right\|_{\frac{2 n}{n+2}}+\left\|d_{g_{\xi}}(\cdot, \xi)^{N-1} \widetilde{W}_{\varepsilon, t, \xi}^{2^{*}-1}\right\|_{\frac{2 n}{n+2}}\right)\left\|\widetilde{\phi}_{\varepsilon, t, \xi}\right\|_{2^{*}}\right) .
\end{array}
$$


In dimension $n=4$, rough estimates give that

$$
\begin{array}{r}
\left\|d_{g_{\xi}}(\cdot, \xi) \nabla \widetilde{W}_{\varepsilon, t, \xi}\right\|_{2}=\mathrm{O}\left(\delta_{\varepsilon}(t) \sqrt{\left|\ln \delta_{\varepsilon}(t)\right|}\right), \quad\left\|d_{g_{\xi}}(\cdot, \xi) \widetilde{W}_{\varepsilon, t, \xi}\right\|_{\frac{2 n}{n+2}}=\mathrm{O}\left(\delta_{\varepsilon}(t)\right), \\
\left\|\widetilde{W}_{\varepsilon, t, \xi}\right\|_{\frac{2 n}{n+2}}=\mathrm{O}\left(\delta_{\varepsilon}(t)\right), \quad \text { and } \quad\left\|d_{g_{\xi}}(\cdot, \xi)^{N-1} \widetilde{W}_{\varepsilon, t, \xi}^{2^{*}-1}\right\|_{\frac{2 n}{n+2}}=\mathrm{O}\left(\delta_{\varepsilon}(t)^{3}\right) .
\end{array}
$$

It follows from (6.71)-(6.72) and from Sobolev's embedding $H_{1}^{2}(M) \hookrightarrow L^{2^{*}}(M)$ that

$$
I_{2, i, \varepsilon, t, \xi}=\mathrm{O}\left(\delta_{\varepsilon}(t) \sqrt{\left|\ln \delta_{\varepsilon}(t)\right|}\left\|\widetilde{\phi}_{\varepsilon, t, \xi}\right\|_{1,2}\right) .
$$

It remains to estimate $I_{3, i, \varepsilon, t, \xi}$ and $I_{4, i, \varepsilon, t, \xi}$. Clearly, we get that

$$
I_{3, i, \varepsilon, t, \xi}=\mathrm{O}\left(\left\|\widetilde{\phi}_{\varepsilon, t, \xi}\right\|_{1,2}^{2}\right) \text {. }
$$

Similarly as in (4.17), we get that

$$
I_{4, i, \varepsilon, t, \xi}=\mathrm{O}\left(\left\|\widetilde{\phi}_{\varepsilon, t, \xi}\right\|_{2^{*}}^{2}+\left\|\widetilde{\phi}_{\varepsilon, t, \xi}\right\|_{2^{*}}^{2^{*}}\right) .
$$

Since $\left\|\phi_{\varepsilon, t, \xi}\right\|_{1,2}=\mathrm{O}\left(\left\|\phi_{\varepsilon, t, \xi}\right\|_{L_{g}}\right)=\mathrm{O}\left(\varepsilon \delta_{\varepsilon}(t)\right)$, by (6.73) - 6.75$)$, and by Sobolev's embedding $H_{1}^{2}(M) \hookrightarrow L^{2^{*}}(M)$, we get that

$$
I_{2, i, \varepsilon, t, \xi}+I_{3, i, \varepsilon, t, \xi}+I_{4, i, \varepsilon, t, \xi}=\mathrm{O}\left(\sqrt{\varepsilon} \delta_{\varepsilon}(t)^{2}\right)=\mathrm{o}\left(\delta_{\varepsilon}(t)^{2}\right)
$$

as $\varepsilon \rightarrow 0$. Finally, it follows from (6.66), (6.68), (6.69), and (6.76) that (4.14) is $C^{1}$-uniform with respect to $\xi$.

\section{APPENDIX}

In this appendix, first, we state the following result which is due to Lee-Parker [27].

Lemma A.1. For any $\xi \in M$, let $g_{\xi}$ be as in Section 0 and $G_{g_{\xi}}$ be the Green's function of the conformal Laplacian with respect to $g_{\xi}$. Then the following expansions do hold:

(i) when $n=4,5$ or $(M, g)$ is l.c.f.,

$$
G_{g_{\xi}}\left(\exp _{\xi} y, \xi\right)=\beta_{n}^{-1}|y|^{2-n}+A_{\xi}+\mathrm{O}(|y|),
$$

(ii) when $n=6$,

$$
G_{g_{\xi}}\left(\exp _{\xi} y, \xi\right)=\beta_{6}^{-1}\left(|y|^{-4}-\frac{1}{1440}\left|\operatorname{Weyl}_{g}(\xi)\right|_{g}^{2} \ln |y|\right)+\mathrm{O}(|y|)
$$

(iii) when $n \geq 7$,

$$
\begin{array}{r}
G_{g_{\xi}}\left(\exp _{\xi} y, \xi\right)=\beta_{n}^{-1}|y|^{2-n}\left(1+\frac{\alpha_{n}|y|^{2}}{12(n-4)}\left(\frac{1}{12(n-6)}\left|\operatorname{Weyl}_{g}(\xi)\right|_{g}^{2}|y|^{2}\right.\right. \\
\left.\left.-\partial_{y_{i} y_{j}}\left[\operatorname{Scal}_{g_{\xi}} \circ \exp _{\xi}\right](0) y_{i} y_{j}\right)\right)+\mathrm{O}\left(|y|^{7-n}\right)
\end{array}
$$

$C^{1}$-uniformly with respect to $y$ and $\xi$, where Weyl $g$ is the Weyl curvature tensor with respect to $g$, and for any $\xi \in M, \mathrm{Scal}_{g_{\xi}}$ is the scalar curvature with respect to $g_{\xi}$.

Proof. We refer to Lee-Parker [27, Lemma 6.4]. The only point which is not discussed in [27] is the $C^{1}$-uniformity of the expansions with respect to $\xi$. This point follows from standard arguments.

Finally, let us estimate the first derivatives of the geodesic distance in the following result. 
Lemma A.2. For any $\xi \in M$, let $g_{\xi}$ be as in Section Q and $d_{g_{\xi}}$ be the geodesic distance with respect to $g_{\xi}$. Then there holds

$$
\left.\nabla\left(d_{\exp _{\xi} \eta}\left(\exp _{\xi} y, \exp _{\xi} \eta\right)\right)\right|_{\eta=0}=-\frac{y}{|y|}+\mathrm{O}\left(|y|^{2}\right)
$$

uniformly with respect to $\xi \in M$ and $y \in T_{\xi} M \cong \mathbb{R}^{n}, y \neq 0,|y| \ll 1$.

Proof. By compactness of $M$ and since $g_{\xi}$ is smooth with respect to $\xi$, we get that there exists a positive real number $r_{0}$ such that $r_{0}<i_{g_{\xi}}$ for all $\xi \in M$, where $i_{g_{\xi}}$ is the injectivity radius of the manifold $\left(M, g_{\xi}\right)$. For any $\xi \in M$, we let $B_{0}\left(r_{0}\right)$ be the ball in $T_{\xi} M \cong \mathbb{R}^{n}$ of center 0 and radius $r_{0}$. For any $\xi \in M$ and $y \in B_{0}\left(r_{0}\right) \backslash\{0\}$, we write that

$$
\left.\nabla\left(d_{g_{\exp \eta}}\left(\exp _{\xi} y, \exp _{\xi} \eta\right)\right)\right|_{\eta=0}=\left.\nabla\left(d_{\exp _{\xi} \eta}\left(\exp _{\xi} y, \xi\right)\right)\right|_{\eta=0}+\left.\nabla\left(d_{g_{\xi}}\left(\exp _{\xi} y, \exp _{\xi} \eta\right)\right)\right|_{\eta=0} \text {. }
$$

We begin with estimating the first term in the right hand side of (A.2). Since we have chosen $\Lambda_{\xi}$ so that $\Lambda_{\xi}(\xi)=1$ and $\nabla \Lambda_{\xi}(\xi)=0$, we get that

$$
\Lambda_{\exp _{\xi} \eta}\left(\exp _{\xi} y\right)=\Lambda_{\xi}\left(\exp _{\xi} y\right)\left(1+\mathrm{O}\left(|y|^{2}+|\eta|^{2}\right)\right)
$$

uniformly with respect to $\xi \in M$ and $y, \eta \in B_{0}\left(r_{0}\right)$. It follows that

$$
d_{\exp ^{\eta} \eta}\left(\exp _{\xi} y, \xi\right)=d_{g_{\xi}}\left(\exp _{\xi} y, \xi\right)\left(1+\mathrm{O}\left(|y|^{2}+|\eta|^{2}\right)\right)=|y|+\mathrm{O}\left(|y|^{3}+|y||\eta|^{2}\right),
$$

and thus we get that

$$
\left.\nabla\left(d_{\exp _{\xi} \eta}\left(\exp _{\xi} y, \xi\right)\right)\right|_{\eta=0}=\mathrm{O}\left(|y|^{2}\right)
$$

uniformly with respect to $\xi \in M$ and $y \in B_{0}\left(r_{0}\right)$. Now, we estimate the second term in the right hand side of (A.2). For any $\xi \in M$ and $\eta \in T_{\xi} M$, we let $T_{\xi, \exp _{\xi} \eta} M$ and $\exp _{\xi, \exp _{\xi} \eta}$ be the respective tangent space and exponential map at $\exp _{\xi} \eta$ with respect to $g_{\xi}$. For any $\eta \in B_{0}\left(r_{0}\right)$, we identify $T_{\xi, \exp _{\xi} \eta} M$ with $\mathbb{R}^{n}$ thanks to a local orthonormal frame, parallel at $\xi$. For any $i=1, \ldots, n, \xi \in M$, and $y \in B_{0}\left(r_{0}\right) \backslash\{0\}$, we get that

$$
\left.\frac{d}{d \eta_{i}}\left(d_{g_{\xi}}\left(\exp _{\xi} y, \exp _{\xi} \eta\right)\right)\right|_{\eta=0}=\left.\sum_{j=1}^{n} \frac{y_{j}}{|y|} \frac{d}{d \eta_{i}}\left(\exp _{\xi, \exp _{\xi} \eta}^{-1} \exp _{\xi} y\right)_{j}\right|_{\eta=0} .
$$

We claim that for any $i, j=1, \ldots, n$, there holds

$$
\left.\frac{d}{d \eta_{i}}\left(\exp _{\xi, \exp _{\xi} \eta}^{-1} \exp _{\xi} y\right)_{j}\right|_{\eta=0}=-\delta_{i j}+\mathrm{O}\left(|y|^{2}\right)
$$

uniformly with respect to $\xi \in M$ and $y \in B_{0}\left(r_{0}\right)$, where the $\delta_{i j}$ 's are the Kronecker symbols. We prove this claim. For any $j=1, \ldots, n, \xi \in M$ and $\eta, y \in B_{0}\left(r_{0}\right)$, we define

$$
\mathcal{E}_{j, \xi}(y, \eta):=\left(\exp _{\xi, \exp _{\xi} \eta}^{-1} \exp _{\xi} y\right)_{j} .
$$

Clearly, $\mathcal{E}_{j, \xi}(y, \eta)$ is smooth with respect to $\xi, y$, and $\eta$. In order to prove the Taylor expansion (A.5), we compute the first and second order derivatives of $\mathcal{E}_{j, \xi}(y, \eta)$ with respect to $y$ and $\eta$. Since the frame is parallel at $\xi$, we get that $\mathcal{E}_{j, \xi}(0, \eta)=-\eta_{j}$ for all $\eta \in B_{0}\left(r_{0}\right)$. Differentiating this equation gives that

$$
\partial_{\eta_{i}} \mathcal{E}_{j, \xi}(0,0)=-\delta_{i j} \quad \text { and } \quad \partial_{\eta_{i} \eta_{k}} \mathcal{E}_{j, \xi}(0,0)=0
$$

for all $i, j, k=1, \ldots, n$. We also remark that $\mathcal{E}_{j, \xi}(y, 0)=y_{j}$ for all $y \in B_{0}\left(r_{0}\right)$, and thus we get that

$$
\partial_{y_{i}} \mathcal{E}_{j, \xi}(0,0)=\delta_{i j} \quad \text { and } \quad \partial_{y_{i} y_{k}} \mathcal{E}_{j, \xi}(0,0)=0
$$


for all $i, j, k=1, \ldots, n$. As a third equation, we find $\mathcal{E}_{j, \xi}(y, y)=0$ for all $y \in B_{0}\left(r_{0}\right)$. Differentiating this equation and using (A.6) and (A.7), we find that

$$
\partial_{\eta_{i} y_{k}} \mathcal{E}_{j, \xi}(0,0)=-\frac{1}{2}\left(\partial_{\eta_{i} \eta_{k}} \mathcal{E}_{j, \xi}(0,0)+\partial_{y_{i} y_{k}} \mathcal{E}_{j, \xi}(0,0)\right)=0
$$

for all $i, j, k=1, \ldots, n$. (A.5) follows from (A.6) and (A.8). Finally, (A.1) follows from (A.2) - A.5 . This ends the proof of Lemma A.2.

\section{REFERENCES}

[1] A. Ambrosetti and A. Malchiodi, A multiplicity result for the Yamabe problem on $\mathbb{S}^{n}$, J. Funct. Anal. 168 (1999), no. 2, 529-561.

[2] T. Aubin, Problèmes isopérimétriques et espaces de Sobolev, J. Differential Geom. 11 (1976), no. 4, 573598 (French).

[3] _ Equations différentielles non linéaires et problème de Yamabe concernant la courbure scalaire, J. Math. Pures Appl. (9) 55 (1976), no. 3, 269-296.

[4] A. Bahri, Y.-Y. Li, and O. Rey, On a variational problem with lack of compactness: the topological effect of the critical points at infinity, Calc. Var. Partial Differential Equations 3 (1995), no. 1, 67-93.

[5] M. Ben Ayed, K. El Mehdi, O. Rey, and M. Grossi, A nonexistence result of single peaked solutions to a supercritical nonlinear problem, Commun. Contemp. Math. 5 (2003), no. 2, 179-195.

[6] G. Bianchi and H. Egnell, A note on the Sobolev inequality, J. Funct. Anal. 100 (1991), no. 1, 18-24.

[7] M. Berti and A. Malchiodi, Non-compactness and multiplicity results for the Yamabe problem on $\mathbb{S}^{n}$, J. Funct. Anal. 180 (2001), no. 1, 210-241.

[8] S. Brendle, Blow-up phenomena for the Yamabe equation, J. Amer. Math. Soc. 21 (2008), no. 4, 951-979.

[9] S. Brendle and F. C. Marques, Blow-up phenomena for the Yamabe equation. II, J. Differential Geom. 81 (2009), no. 2, 225-250.

[10] H. Brézis and L. Nirenberg, Positive solutions of nonlinear elliptic equations involving critical Sobolev exponents, Comm. Pure Appl. Math. 36 (1983), no. 4, 437-477.

[11] J.-G. Cao, The existence of generalized isothermal coordinates for higher-dimensional Riemannian manifolds, Trans. Amer. Math. Soc. 324 (1991), no. 2, 901-920.

[12] W.-Y. Chen, J.-C. Wei, and S.-S. Yan, Infinitely many solutions for the Schrödinger equations in $\mathbb{R}^{n}$ with critical growth, J. Differential Equations 252 (2012), no. 3, 2425-2447.

[13] O. Druet, From one bubble to several bubbles: the low-dimensional case, J. Differential Geom. 63 (2003), no. $3,399-473$.

[14] _ Compactness for Yamabe metrics in low dimensions, Int. Math. Res. Not. 23 (2004), 1143-1191.

[15] O. Druet and E. Hebey, Blow-up examples for second order elliptic PDEs of critical Sobolev growth, Trans. Amer. Math. Soc. 357 (2005), no. 5, 1915-1929 (electronic).

[16] _ Elliptic equations of Yamabe type, Int. Math. Res. Surv. 1 (2005), 1-113.

[17] _ Stability for strongly coupled critical elliptic systems in a fully inhomogeneous medium, Anal. PDE 2 (2009), no. 3, 305-359.

[18] O. Druet, E. Hebey, and F. Robert, Blow-up theory for elliptic PDEs in Riemannian geometry, Mathematical Notes, vol. 45, Princeton University Press, Princeton, NJ, 2004.

[19] O. Druet, E. Hebey, and J. Vétois, Bounded stability for strongly coupled critical elliptic systems below the geometric threshold of the conformal Laplacian, J. Funct. Anal. 258 (2010), no. 3, 999-1059.

[20] P. Esposito, A. Pistoia, and J. Vétois, Blow-up solutions for linear perturbations of the Yamabe equation. Preprint.

[21] I. Fonseca and W. Gangbo, Degree theory in analysis and applications, Oxford Lecture Series in Mathematics and its Applications, vol. 2, The Clarendon Press Oxford University Press, New York, 1995. Oxford Science Publications.

[22] M. Günther, Conformal normal coordinates, Ann. Global Anal. Geom. 11 (1993), no. 2, 173-184.

[23] Z.-C. Han, Asymptotic approach to singular solutions for nonlinear elliptic equations involving critical Sobolev exponent, Ann. Inst. H. Poincaré Anal. Non Linéaire 8 (1991), no. 2, 159-174.

[24] E. Hebey and M. Vaugon, From best constants to critical functions, Math. Z. 237 (2001), no. 4, 737-767.

[25] E. Hebey and J.-C. Wei, Resonant states for the static Klein-Gordon-Maxwell-Proca system, Math. Res. Lett. To appear. 
[26] M. A. Khuri, F. C. Marques, and R. M. Schoen, A compactness theorem for the Yamabe problem, J. Differential Geom. 81 (2009), no. 1, 143-196.

[27] J. M. Lee and T. H. Parker, The Yamabe problem, Bull. Amer. Math. Soc. (N.S.) 17 (1987), no. 1, $37-91$.

[28] J. Lelong-Ferrand, Transformations conformes et quasi-conformes des variétés riemanniennes compactes (démonstration de la conjecture de A. Lichnerowicz), Acad. Roy. Belg. Cl. Sci. Mém. Coll. (2) 39 (1971), no. 5, 44 (French).

[29] Y.-Y. Li, On a singularly perturbed elliptic equation, Adv. Differential Equations 2 (1997), no. 6, 955-980.

[30] Y.-Y. Li and L. Zhang, Compactness of solutions to the Yamabe problem. II, Calc. Var. Partial Differential Equations 24 (2005), no. 2, 185-237.

[31] Y.Y. Li and L. Zhang, Compactness of solutions to the Yamabe problem. III, J. Funct. Anal. 245 (2007), no. 2, 438-474.

[32] Y.-Y. Li and M.-J. Zhu, Yamabe type equations on three-dimensional Riemannian manifolds, Commun. Contemp. Math. 1 (1999), no. 1, 1-50.

[33] F. C. Marques, A priori estimates for the Yamabe problem in the non-locally conformally flat case, J. Differential Geom. 71 (2005), no. 2, 315-346.

[34] M. Musso and A. Pistoia, Double blow-up solutions for a Brezis-Nirenberg type problem, Commun. Contemp. Math. 5 (2003), no. 5, 775-802.

[35] _ Multispike solutions for a nonlinear elliptic problem involving the critical Sobolev exponent, Indiana Univ. Math. J. 51 (2002), no. 3, 541-579.

[36] M. Obata, The conjectures on conformal transformations of Riemannian manifolds, J. Differential Geometry $6(1971 / 72), 247-258$.

[37] A. Pistoia and O. Rey, Multiplicity of solutions to the supercritical Bahri-Coron's problem in pierced domains, Adv. Differential Equations 11 (2006), no. 6, 647-666.

[38] S. I. Pohožaev, On the eigenfunctions of the equation $\Delta u+\lambda f(u)=0$, Soviet Math. Dokl. 6 (1965), $1408-1411$.

[39] O. Rey, Proof of two conjectures of H. Brézis and L. A. Peletier, Manuscripta Math. 65 (1989), no. 1, 19-37.

[40] ㄴ The role of the Green's function in a nonlinear elliptic equation involving the critical Sobolev exponent, J. Funct. Anal. 89 (1990), no. 1, 1-52.

[41] E. Rodemich, The Sobolev inequalities with best possible constants. Analysis Seminar at California Institute of Technology (1966).

[42] R. M. Schoen, Conformal deformation of a Riemannian metric to constant scalar curvature, J. Differential Geom. 20 (1984), no. 2, 479-495.

[43] - Variational theory for the total scalar curvature functional for Riemannian metrics and related topics, Topics in calculus of variations (Montecatini Terme, 1987), Lecture Notes in Math., vol. 1365, Springer, Berlin, 1989, pp. 120-154.

[44] - On the number of constant scalar curvature metrics in a conformal class, Differential geometry, Pitman Monogr. Surveys Pure Appl. Math., vol. 52, Longman Sci. Tech., Harlow, 1991, pp. 311-320.

[45] R. M. Schoen and S.-T. Yau, On the proof of the positive mass conjecture in general relativity, Comm. Math. Phys. 65 (1979), no. 1, 45-76.

[46] — Conformally flat manifolds, Kleinian groups and scalar curvature, Invent. Math. 92 (1988), no. 1, 47-71.

[47] G. Talenti, Best constant in Sobolev inequality, Ann. Mat. Pura Appl. (4) 110 (1976), 353-372.

[48] N. S. Trudinger, Remarks concerning the conformal deformation of Riemannian structures on compact manifolds, Ann. Scuola Norm. Sup. Pisa (3) 22 (1968), 265-274.

[49] H. Yamabe, On a deformation of Riemannian structures on compact manifolds, Osaka Math. J. 12 (1960), 21-37. 
Pierpaolo Esposito, Dipartimento di Matematica, Università degli Studi "Roma Tre", Largo S. Leonardo Murialdo 1, 00146 Roma, Italy

E-mail address: esposito@mat.uniroma3.it

Angela Pistoia, Dipartimento SBai, Università di Roma "La Sapienza", via Antonio Scarpa 16, 00161 Roma, Italy

E-mail address: pistoia@dmmm.uniroma1.it

Jérôme Vétois, Université de Nice - Sophia Antipolis, Laboratoire J.-A. Dieudonné, UmR CNRS-UnS 7351, Parc Valrose, 06108 Nice Cedex 2, France

E-mail address: vetois@unice.fr 